\title{
Tandem Sakurai-Aldol Addition Reactions as a Route to Structurally Complex Carbocycles
}

Benjamin D. Stevens and Scott G. Nelson*

Department of Chemistry, University of Pittsburgh, Pittsburgh, PA 15260

\section{TABLE OF CONTENTS}

Compound 4c and General Procedure A for Preparing Diallylethers 5

Compounds 5a-c

S2-S3

General Procedure B for ICR Reactions

S3

Compounds 7a-c

S3-S4

General Procedure C for Preparing Allylic and Homoallylic Alcohols C

Compounds 10a-c

General Procedure D for Preparing Unsaturated Ketones 8 and 9 
General Information: Unless otherwise stated all reactions were performed in dry glassware under an atmosphere of oxygen free nitrogen using standard inert atmosphere techniques for the manipulation of solvents and reagents. Anhydrous solvents were obtained by passing through successive alumina columns on a solvent purification system. $\quad\left[\operatorname{IrCl}\left(\mathrm{C}_{8} \mathrm{H}_{14}\right)_{2}\right]_{2}{ }^{1}$ and $\mathrm{PCy}_{3}$ were stored and weighed out in a glove box. Chemical shifts are reported relative to residual $\mathrm{CHCl}_{3}(7.27 \mathrm{ppm})$ for ${ }^{1} \mathrm{H}$, and $\underline{\mathbf{C D C l}}{ }_{3}(77.0 \mathrm{ppm})$ or $\underline{\mathrm{CD}}_{3} \mathrm{CN}$ (1.39 ppm) for ${ }^{13} \mathrm{C} \mathrm{NMR}$ spectra. Internal temperature of microwave reactions was monitored by integrated infrared detector.

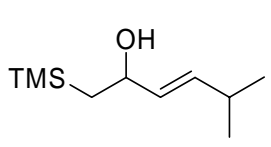

(E)-5-Methyl-1-(trimethylsilyl)hex-3-en-2-ol (4c): In a $250 \mathrm{~mL}$ flask equipped with a dry ice-cooled condenser was added $3.7 \mathrm{~g}$ of $\mathrm{Mg}(0)(0.15 \mathrm{~mol})$ (activated by mechanical agitation) and $75 \mathrm{~mL}$ of $\mathrm{Et}_{2} \mathrm{O}$. A solution of $10 \mathrm{mg}$ of $\mathrm{I}_{2}$ in $1 \mathrm{~mL}$ of $\mathrm{BrCH}_{2} \mathrm{CH}_{2} \mathrm{Br}$ was added to the stirring suspension. Once the reaction had initiated (indicated by a brown-to-clear/white color change), a solution of $11 \mathrm{~mL}$ of chloromethyltrimethylsilane $(9.4 \mathrm{~g}, 76 \mathrm{mmol})$ in $10 \mathrm{~mL}$ of $\mathrm{Et}_{2} \mathrm{O}$ was added at a rate to maintain a gentle reflux. Once addition was complete, the reaction was stirred at ambient temperature for $1 \mathrm{~h}$ and the resulting solution of trimethylsilylmethylmagnesium chloride was added slowly via syringe to a -78 ${ }^{\circ} \mathrm{C}$ solution of $5.0 \mathrm{~g}$ of 4-methyl-2-pentenal $(5.9 \mathrm{~mL}, 51 \mathrm{mmol})$ in $150 \mathrm{~mL}$ of $\mathrm{Et}_{2} \mathrm{O}$. After stirring $30 \mathrm{~min}$ at -78 ${ }^{\circ} \mathrm{C}$, the reaction was quenched at $-78{ }^{\circ} \mathrm{C}$ with sat. aq. $\mathrm{NH}_{4} \mathrm{Cl}$ and the resulting biphasic mixture was warmed to ambient temperature. The aqueous layer was extracted with $\mathrm{Et}_{2} \mathrm{O}(3 \mathrm{x})$, the combined organics were dried $\left(\mathrm{MgSO}_{4}\right)$ and concentrated. The resulting crude product mixture was purified by Kughelrohr distillation $\left(43^{\circ} \mathrm{C}\right.$, 200 mtorr) to afford $7.7 \mathrm{~g}$ (81\%) of the title compound as a colorless oil. IR (thin film) 3358, 2957, 1670, 1248, 971, $839 \mathrm{~cm}^{-1} ;{ }^{1} \mathrm{H}$ NMR $\left(300 \mathrm{MHz}, \mathrm{CDCl}_{3}\right): \delta 5.59(\mathrm{dd}, J=15.4,6.3 \mathrm{~Hz}, 1 \mathrm{H}), 5.42(\mathrm{dd}, J=15.4,7.4 \mathrm{~Hz}, 1 \mathrm{H})$, $4.28-4.20(\mathrm{~m}, 1 \mathrm{H}), 2.36-2.23(\mathrm{~m}, 1 \mathrm{H}), 1.35(\mathrm{~d}, J=3.7 \mathrm{~Hz}, 1 \mathrm{H}), 1.03(\mathrm{dd}, J=14.1,6.2 \mathrm{~Hz}, 1 \mathrm{H}), 1.00(\mathrm{~d}, J=$ $6.8 \mathrm{~Hz}, 6 \mathrm{H}), 0.92(\mathrm{dd}, J=14.1,8.5 \mathrm{~Hz}, 1 \mathrm{H}), 0.03(\mathrm{~s}, 9 \mathrm{H}) ;{ }^{13} \mathrm{C} \mathrm{NMR}\left(75 \mathrm{MHz}, \mathrm{CDCl}_{3}\right)$ : $\delta-1.0,22.0,22.1,26.7,30.3,71.3,132.2,137.6$; MS (EI) $\mathrm{m} / \mathrm{z} 185\left(\mathrm{M}^{+}-\mathrm{H}\right), 169,143,73$; HRMS (EI) $\mathrm{m} / \mathrm{z}$ calculated for $\mathrm{C}_{10} \mathrm{H}_{21} \mathrm{OSi}\left(\mathrm{M}^{+}-\mathrm{H}\right):$ 185.1362, found 185.1363 .

General Procedure A for Preparing Diallylethers 5: The diallyl ethers 5a-c were prepared using a modification of the literature procedure. ${ }^{2}$ To a solution of the allylic alcohol $7(20 \mathrm{mmol})$ in $13 \mathrm{~mL}$ of THF was added $13 \mathrm{~mL}$ of a $1.0 \mathrm{M}$ hexanes solution of $\mathrm{Et}_{2} \mathrm{Zn}(13 \mathrm{mmol})$. The resulting solution was stirred at ambient temperature for $1 \mathrm{~h}$. Into a separate flask were weighed $0.22 \mathrm{~g}(1.0 \mathrm{mmol})$ of $\mathrm{Pd}(\mathrm{OAc})_{2}$ and $1.3 \mathrm{~g}(5.0 \mathrm{mmol})$ of $\mathrm{PPh}_{3}$. The flask was purged with $\mathrm{N}_{2}$ and $26 \mathrm{~mL}$ of THF was added and this mixture was stirred vigorously for 10 min whereupon $3.2 \mathrm{~mL}(3.0 \mathrm{~g}, 30 \mathrm{mmol})$ of allyl acetate was added in a single portion and this mixture was stirred an additional $10 \mathrm{~min}$. The zinc alkoxide solution prepared above was added to this catalyst solution via cannula and the resulting reaction mixture was stirred for the indicated time. The reaction undergoes a color change from yellow to orange and returns to the original yellow color once the reaction is complete. The solvent was removed in vacuo and the salts were precipitated by adding $\mathrm{Et}_{2} \mathrm{O}$ and removed by filtering through florsil. The crude product mixture was purified as indicated.

(E)-(2-Allyloxypent-3-enyl)trimethylsilane (5a): ${ }^{3}$ General Procedure A was followed employing $8.0 \mathrm{~g}(51 \mathrm{mmol})$ of $(E)-1$-trimethylsilanylpent-3-en-2-ol ${ }^{4}$ and a final reaction time of $168 \mathrm{~h}$. Purifying the crude product mixture by Kughelrohr distillation $\left(35^{\circ} \mathrm{C}, 150 \mathrm{mtorr}\right)$ and MPLC chromatography $\left(39: 1\right.$ hexanes/ $\left.\mathrm{Et}_{2} \mathrm{O}\right)$ yielded $8.0 \mathrm{~g}(79 \%)$ of the title compound as a colorless oil. ${ }^{1} \mathrm{H}$ NMR (300 MHz, $\left.\mathrm{CDCl}_{3}\right): \delta 5.96-5.84(\mathrm{~m}, 1 \mathrm{H}), 5.55(\mathrm{dq}, J=15,6.4 \mathrm{~Hz}, 1 \mathrm{H}), 5.28(\mathrm{ddq}, J=15,8.5,1.6 \mathrm{~Hz}, 1 \mathrm{H})$, $5.23(\mathrm{dq}, J=17,1.7 \mathrm{~Hz}, 1 \mathrm{H}), 5.12(\mathrm{dq}, J=10,1.3 \mathrm{~Hz}, 1 \mathrm{H}), 3.97(\mathrm{ddt}, J=13,5.3,1.5 \mathrm{~Hz}, 1 \mathrm{H}), 3.80-3.70(\mathrm{~m}$, 2H), $1.70(\mathrm{dd}, J=6.5,1.5 \mathrm{~Hz}, 1 \mathrm{H}), 1.06(\mathrm{dd}, J=14,6.7 \mathrm{~Hz}, 1 \mathrm{H}), 0.85(\mathrm{dd}, J=14,8.0 \mathrm{~Hz}, 1 \mathrm{H}), 0.01(\mathrm{~s}, 9 \mathrm{H})$.

\footnotetext{
${ }^{1}$ Onderdelinden, A. L.; van der Ent, A. Inorg. Chim. Acta 1972, 6, 420.

${ }^{2}$ Kim, H.; Lee, C. Org. Lett. 2002, 4, 4369.

${ }^{3}$ Nelson, S. G.; Bungard, C. J.; Wang, K. J. Am. Chem. Soc. 2003, 125, 13000.

${ }^{4}$ Taylor, R. T.; Galloway, J. G. J. Organomet. Chem. 1981, 220, 295.
} 


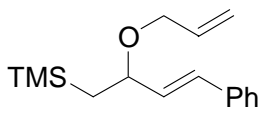

(E)-(2-Allyloxy-4-phenylbut-3-enyl)trimethylsilane (5b): ${ }^{2}$ General Procedure A was followed employing $5.0 \mathrm{~g}(23 \mathrm{mmol})$ of $(E)$-4-phenyl-1-trimethylsilanylbut-3-en-2-ol ${ }^{5}$ and a final reaction time of $48 \mathrm{~h}$. Purifying the crude product mixture by MPLC chromatography (39:1 hexanes/Et $2 \mathrm{O})$ affording $3.1 \mathrm{~g}(52 \%)$ of the title compound as a colorless oil. ${ }^{1} \mathrm{H} \mathrm{NMR}\left(300 \mathrm{MHz}, \mathrm{CDCl}_{3}\right)$ : $\delta$ 7.61-7.24 (m, 5H), $6.04(\mathrm{dd}, J=16,8.3 \mathrm{~Hz}, 1 \mathrm{H}), 5.92(\mathrm{~m}, 1 \mathrm{H}), 5.26$ (ddd, $J=17,3.5,1.6 \mathrm{~Hz}, 1 \mathrm{H}), 5.15$ (ddd, $J$ $=10,3.2,1.3 \mathrm{~Hz}, 1 \mathrm{H}), 4.10-3.95(\mathrm{~m}, 2 \mathrm{H}), 3.83(\mathrm{ddt}, J=13,5.9,1.4 \mathrm{~Hz}, 1 \mathrm{H}), 1.17(\mathrm{dd}, J=14,7.2 \mathrm{~Hz}, 1 \mathrm{H}), 0.96$ (dd, $J=14,7.5 \mathrm{~Hz}, 1 \mathrm{H}), 0.28(\mathrm{~s}, 9 \mathrm{H})$.

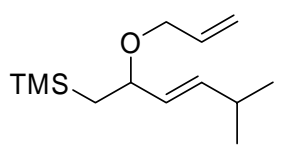

(E)-2-(Allyloxy-5-methylhex-3-enyl)trimethylsilane (5c): The general Procedure A was followed employing $5.1 \mathrm{~g}(27 \mathrm{mmol})$ of alcohol $7 \mathrm{~b}$ and a final reaction time of $168 \mathrm{~h}$. Purifying the crude product mixture by Kughelrohr distillation $\left(35^{\circ} \mathrm{C}, 150 \mathrm{mtorr}\right)$ followed by MPLC chromatography $\left(39: 1\right.$ hexanes/ $\left.\mathrm{Et}_{2} \mathrm{O}\right)$ yielded $5.7 \mathrm{~g}(93 \%)$ of the title compound as a colorless oil. IR (thin film) 2957, 1670, 1650, 1247, 1071, 972, $838 \mathrm{~cm}^{-1} ;{ }^{1} \mathrm{H} \mathrm{NMR}\left(300 \mathrm{MHz}, \mathrm{CDCl}_{3}\right): \delta 5.91$, (dddd, $J=17.2,10.4,5.8,5.3 \mathrm{~Hz}, 1 \mathrm{H}), 5.51(\mathrm{dd}, J=15.5,6.5 \mathrm{~Hz}, 1 \mathrm{H}), 5.28-5.17$ (m, 2H), 5.14 (ddt, $J=9.1,1.9$, $1.3 \mathrm{~Hz}, 1 \mathrm{H}), 3.98$ (ddt, $J=14.2,5.3,1.5 \mathrm{~Hz}, 1 \mathrm{H}), 3.80-3.72(\mathrm{~m}, 2 \mathrm{H}), 2.39-2.23(\mathrm{~m}, 1 \mathrm{H}), 1.08(\mathrm{dd}, J=14.3,6.5$ $\mathrm{Hz}, 1 \mathrm{H}), 0.88(\mathrm{dd}, J=14.3,8.3 \mathrm{~Hz}, 1 \mathrm{H}), 1.01(\mathrm{~d}, J=6.8 \mathrm{~Hz}, 3 \mathrm{H}) ;{ }^{13} \mathrm{C} \mathrm{NMR}\left(75 \mathrm{MHz}, \mathrm{CDCl}_{3}\right): \delta-0.6,22.1,22.4$, 25.1, 30.6, 68.4, 78.5, 116.2, 129.5, 135.5, 140.0; MS (EI) m/z $183\left(\mathrm{M}^{+}-{ }^{i} \mathrm{Pr}\right), 169,139,73$; HRMS (EI) m/z calculated for $\mathrm{C}_{10} \mathrm{H}_{19} \mathrm{OSi}\left(\mathrm{M}^{+}-{ }^{i} \mathrm{Pr}\right): 183.1205$, found 183.1200 .

General Procedure B for ICR Reactions: ICR reactions were performed using a modification of the literature procedure. ${ }^{3} \quad$ Large-scale olefin isomerizations $(\geq 2.0 \mathrm{~g})$ proved to be moderately exothermic; performing the initial allyl ether isomerization at $0{ }^{\circ} \mathrm{C}$ generally afforded improved $E: Z$ vinyl ether ratios. Also, using microwave irradiation for the thermal $[3,3]$ sigmatropic rearrangements was found to require shorter reaction times relative to reactions performed using conventional heating.

A solution of $6.8 \mathrm{mg}(0.020 \mathrm{mmol})$ of $\mathrm{NaBPh}_{4}$ in $1.5 \mathrm{ml}$ of $\mathrm{CH}_{2} \mathrm{Cl}_{2} /$ acetone $(25: 1)$ was added to $9.0 \mathrm{mg}$ $(0.010 \mathrm{mmol})$ of $\left[\mathrm{IrCl}\left(\mathrm{C}_{8} \mathrm{H}_{14}\right)_{2}\right]_{2}$ and $17 \mathrm{mg}$ of $(0.060 \mathrm{mmol}) \mathrm{PCy}_{3}$ in $1.5 \mathrm{~mL}$ of anhydrous $\mathrm{CH}_{2} \mathrm{Cl}_{2}$ and the resulting yellow solution was stirred for $30 \mathrm{~min}$ at ambient temperature then cooled to $0{ }^{\circ} \mathrm{C}$. The diallyl ether 5 $(2.0 \mathrm{mmol})$ was added dropwise to the $0{ }^{\circ} \mathrm{C}$ catalyst solution and the reaction was stirred for $15-30$ min while warming to ambient temperature. To the resulting reaction mixture was added $16 \mathrm{mg}(0.060 \mathrm{mmol}) \mathrm{of}^{\mathrm{PPh}}$ whereupon this solution was transferred to a microwave reaction tube and irradiated at $100^{\circ} \mathrm{C}, 150 \mathrm{~W}$ for the indicated time. Once the reaction was complete, the solvent was evaporated and the residual salts were removed by adding hexanes and filtering the resulting mixture through Celite. Evaporating the solvent afforded the crude aldehyde product that was used in subsequent transformations without purification. For characterization purposes, a small sample of each aldehyde was purified by flash chromatography $\left(\mathrm{SiO}_{2}\right)$ and the spectral data for these purified materials is provided. Diastereomer ratios were determined for the crude aldehydes 7 by integrating the aldehyde $\mathrm{C} \underline{\mathbf{H O}}$ resonances in the ${ }^{1} \mathrm{H}$ NMR $(300 \mathrm{MHz})$; these resonances for the diastereomeric aldehydes were well resolved and readily integrated.

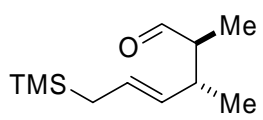

$R^{*}$-(E,2S,3R)-2,3-Dimethyl-6-(trimethylsilyl)hex-4-enal (7a): General Procedure B was followed employing $2.00 \mathrm{~g}(10.1 \mathrm{mmol})$ of diallylether $5 \mathbf{a}$ and a $\mu \mathrm{W}$ reaction time of 45 min to afford $1.96 \mathrm{~g}(98 \%)$ of aldhyde $7 \mathbf{a}$ as a yellow oil (syn:anti $=93: 7)$. IR (thin film) 3018, 2958, 2700, 1727, 1673, 1248, 967, $854 \mathrm{~cm}^{-1}$; ${ }^{1} \mathrm{H}$ NMR (300 MHz, $\left.\mathrm{CDCl}_{3}\right): \delta 9.67(\mathrm{~d}, J=2.2 \mathrm{~Hz}, 1 \mathrm{H})$, $5.46(\mathrm{dtd}, J=15.3,8.0,1.0 \mathrm{~Hz}, 1 \mathrm{H}) 5.21(\mathrm{ddt}, J=15.2,7.7,1.1 \mathrm{~Hz}, 1 \mathrm{H}), 2.54(\mathrm{~m}, 1 \mathrm{H}), 2.31(\mathrm{pd}, J=7.0,2.2 \mathrm{~Hz}$, 1H), 1.44 (dd, $J=8.0,0.9 \mathrm{~Hz}, 2 \mathrm{H}), 1.05(\mathrm{~d}, J=7.0 \mathrm{~Hz}, 3 \mathrm{H}), 1.02(\mathrm{~d}, J=6.9 \mathrm{~Hz}, 3 \mathrm{H}), 0.01(\mathrm{~s}, 9 \mathrm{H}) ;{ }^{13} \mathrm{C}$ NMR $(75$

\footnotetext{
${ }^{5}$ Wang, D.; Chen, D.; Haberman, J. X.; Li, C. Tetrahedron 1988, 54, 5129.
}

-S 3- 
$\left.\mathrm{MHz}, \mathrm{CDCl}_{3}\right): \delta-2.0,10.3,17.4,22.7,37.9,51.4,127.0,131.0,205.6$; MS (EI) $\mathrm{m} / \mathrm{z} 198\left(\mathrm{M}^{+}\right), 183,130,115,73$; HRMS (EI) $\mathrm{m} / \mathrm{z}$ calculated for $\mathrm{C}_{11} \mathrm{H}_{22} \mathrm{OSi}$ : 198.1440 , found 198.1431 .

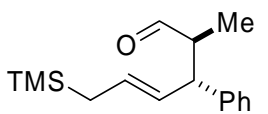

$R^{*}$-(E,2S,3R)-2-Methyl-6-(trimethylsilyl)-3-phenylhex-4-enal (7b): General Procedure B was followed employing $2.0 \mathrm{~g}(7.7 \mathrm{mmol})$ of diallylether $5 \mathbf{b}$ and a $\mu \mathrm{W}$ reaction time of $60 \mathrm{~min}$ to afford $2.0 \mathrm{~g}$ (quant) of aldehyde $\mathbf{7 b}$ as a yellow oil (syn:anti $=92: 8$ ). IR (thin film) $3062,3027,2955,2706,1727,1653,1601,1248,966,851 \mathrm{~cm}^{-1} ;{ }^{1} \mathrm{H}$ NMR $\left(300 \mathrm{MHz}, \mathrm{CDCl}_{3}\right): \delta 9.68(\mathrm{~d}, J=$ $3.3 \mathrm{~Hz}, 1 \mathrm{H}), 7.39-7.16(\mathrm{~m}, 5 \mathrm{H}), 5.54(\mathrm{dt}, J=15.1,6.9 \mathrm{~Hz}, 1 \mathrm{H}), 5.50(\mathrm{dd}, J=15.2,7.3 \mathrm{~Hz}, 1 \mathrm{H}), 3.46(\mathrm{dd}, J=9.5$, $7.5 \mathrm{~Hz}, 1 \mathrm{H}), 2.73(\mathrm{dqd}, J=9.6,6.9,3.2 \mathrm{~Hz}, 1 \mathrm{H}), 1.43(\mathrm{~d}, J=6.7 \mathrm{~Hz}, 2 \mathrm{H}) 0.91(\mathrm{~d}, J=6.9 \mathrm{~Hz}, 3 \mathrm{H}),-0.04(\mathrm{~s}, 9 \mathrm{H})$; ${ }^{13} \mathrm{C}$ NMR $\left(75 \mathrm{MHz}, \mathrm{CD}_{3} \mathrm{CN}\right): \delta-1.7,13.1,23.3,51.7,51.8,127.5,129.0,129.6(2 \mathrm{C}), 130.6,143.8,205.8$; MS (EI) $\mathrm{m} / \mathrm{z} 260\left(\mathrm{M}^{+}\right), 203,130,73$; HRMS (EI) $\mathrm{m} / \mathrm{z}$ calculated for $\mathrm{C}_{16} \mathrm{H}_{24} \mathrm{OSi}: 260.1596$, found 260.1602 .

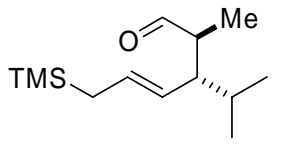

$R^{*}$-(E,2S,3R)-3-Isopropyl-2-methyl-6-(trimethylsilyl)hex-4-enal (7c): General Procedure B was followed employing $2.0 \mathrm{~g}(8.8 \mathrm{mmol})$ of diallylether $5 \mathrm{c}$ and a $\mu \mathrm{W}$ reaction time of $75 \mathrm{~min}$ to afford $2.0 \mathrm{~g}$ (quant.) of aldehyde $7 \mathrm{c}$ as a mixture of $E / Z$ olefin isomers $[E: Z=88: 12$, syn:anti $(E)=91: 9$, syn:anti $(Z)=85: 15)$ as determined by $500{ }^{1} \mathrm{H} \mathrm{MHz}$ NMR [RCh彑 $\delta 9.61$ (E, syn), 9.57 (E, anti), 9.64 (Z, syn), 9.59 (Z, anti)] and analytical GC (CP-Wax $52 \mathrm{CB}(30 \mathrm{~m} \mathrm{x} 0.25 \mathrm{~mm})$, method: $60^{\circ} \mathrm{C}$ for 5.00 min, ramp @ $3^{\circ} \mathrm{C} / \mathrm{min}$ to $250^{\circ} \mathrm{C}$, hold for $\left.20 \mathrm{~min}\right)\left[\left(\mathrm{T}_{\mathrm{r}(\mathrm{E}, \text { syn })}+\mathrm{T}_{\mathrm{r}(\mathrm{E}, \text { anti })}=27.47, \mathrm{~T}_{\mathrm{r}(\mathrm{Z}, \text { syn })}=29.04, \mathrm{~T}_{\mathrm{r}(\mathrm{Z}, \text { anti })}=28.83\right]\right.$. IR (thin film) 2958, 2701, 1727, 1655, 1248, 971, $853 \mathrm{~cm}^{-1} ;{ }^{1} \mathrm{H}$ NMR $\left(300 \mathrm{MHz}, \mathrm{CDCl}_{3}\right): \delta 9.61(\mathrm{~d}, J=3.1 \mathrm{~Hz}$, $1 \mathrm{H}), 5.42(\mathrm{dt}, J=15.1,8.0 \mathrm{~Hz}, 1 \mathrm{H}), 5.07(\mathrm{ddt}, J=15.1,9.7,1.1 \mathrm{~Hz}, 1 \mathrm{H}), 2.47(\mathrm{pd}, J=6.9,3.2 \mathrm{~Hz}, 1 \mathrm{H}), 1.97$ (ddd, $J=9.7,7.7,5.8 \mathrm{~Hz}, 1 \mathrm{H}), 1.89-1.76(\mathrm{~m}, 1 \mathrm{H}), 1.46(\mathrm{dd}, J=7.9,1.0 \mathrm{~Hz}, 2 \mathrm{H}), 1.05(\mathrm{~d}, J=6.9 \mathrm{~Hz}, 3 \mathrm{H}), 0.92$ $(\mathrm{d}, J=6.7 \mathrm{~Hz}, 3 \mathrm{H}), 0.83(\mathrm{~d}, J=6.8 \mathrm{~Hz}, 3 \mathrm{H}), 0.00(\mathrm{~s}, 9 \mathrm{H}) ;{ }^{13} \mathrm{C} \mathrm{NMR}\left(75 \mathrm{MHz}, \mathrm{CDCl}_{3}\right): \delta-1.8,12.5,18.3,21.4$, 23.0, 28.4, 48.1, 51.9, 126.4, 130.1, 205.8; MS (EI) $\mathrm{m} / \mathrm{z} 183\left(\mathrm{M}^{+}{ }^{i} \mathrm{Pr}\right), 169,139,84,73$; HRMS (EI) $\mathrm{m} / \mathrm{z}$ calculated for $\mathrm{C}_{10} \mathrm{H}_{19} \mathrm{OSi}\left(\mathrm{M}^{+}-{ }^{i} \mathrm{Pr}\right): 183.1205$, found 183.1197 .

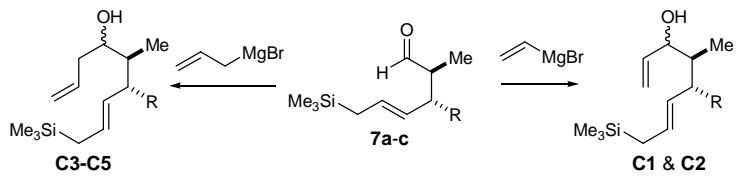

General Procedure C for Preparing Allylic and Homoallylic Alcohols C1-C5. To a $-78{ }^{\circ} \mathrm{C}$ solution of aldehyde 7 (1.0 mmol) in $2.5 \mathrm{~mL}$ of $\mathrm{CH}_{2} \mathrm{Cl}_{2}$ was added a $1.5 \mathrm{mmol}$ of either allylmagnesium bromide $\left(1.0 \mathrm{M}\right.$ in $\left.\mathrm{Et}_{2} \mathrm{O}\right)$ or vinylmagnesium

bromide (1.0 M in THF). After stirring the reaction mixture for 15-60 min, the reaction was quenched by carefully adding sat. aq $\mathrm{NH}_{4} \mathrm{Cl}$ at $-78{ }^{\circ} \mathrm{C}$ then warming the mixture to ambient temperature. The layers were separated and the aqueous layer was extracted with $\mathrm{Et}_{2} \mathrm{O}(3-5 \mathrm{x})$ and the combined organic extracts were dried $\left(\mathrm{MgSO}_{4}\right)$ and concentrated. The resulting crude product mixture contained the desired allylic or homoallylic alcohols as diastereomeric mixtures ( 1:1-3:1). In each case, the diastereomer with the higher $\mathrm{R}_{\mathrm{f}}$ (TLC) was isolated by flash chromatography and fully characterized.

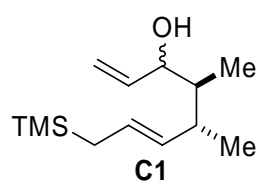

$R^{*}$-(E,3R,4S,5R)-4,5-Dimethyl-8-(trimethylsilyl)octa-1,6-dien-3-ol $+R^{*}$-(E,3S,4S,5R)4,5-Dimethyl-8-(trimethylsilyl)octa-1,6-dien-3-ol (C1): General Procedure C was followed using $1.38 \mathrm{~g}(6.96 \mathrm{mmol})$ of aldehyde $7 \mathrm{a}($ syn:anti $=93: 7)$ and $10.4 \mathrm{~mL}(10.4 \mathrm{mmol})$ of vinylmagnesium bromide. Purifying the crude product mixture by flash chromatography on $\mathrm{SiO}_{2}(25: 1$ hexanes/EtOAc) yielded $792 \mathrm{mg}(50 \%)$ of the title alcohol as a yellow oil. IR (thin film) $3373,3078,2958,1650,1644,1248,851 \mathrm{~cm}^{-1} ;{ }^{1} \mathrm{H}$ NMR $\left(300 \mathrm{MHz}, \mathrm{CDCl}_{3}\right): \delta 5.88$ (ddd, $J=17.2$, $10.5,5.4 \mathrm{~Hz}, 1 \mathrm{H}), 5.43(\mathrm{dt}, J=15.8,8.0 \mathrm{~Hz}, 1 \mathrm{H}), 5.24(\mathrm{dt}, J=17.2,1.6 \mathrm{~Hz}, 1 \mathrm{H}), 5.14(\mathrm{dt}, J=10.5,1.6 \mathrm{~Hz}, 1 \mathrm{H})$, 5.27-5.12 (m, 1H), 4.29-4.23 (m, 1H), 2.17 (sextet, $J=7.1 \mathrm{~Hz}, 1 \mathrm{H}), 1.48(\mathrm{~d}, J=5.3 \mathrm{~Hz}, 1 \mathrm{H}), 1.43(\mathrm{dd}, J=7.8$, $0.9 \mathrm{~Hz}, 2 \mathrm{H}), 1.47-1.33(\mathrm{~m}, 1 \mathrm{H}), 0.97(\mathrm{~d}, J=6.8 \mathrm{~Hz}, 3 \mathrm{H}), 0.88(\mathrm{~d}, J=6.9 \mathrm{~Hz}, 3 \mathrm{H}), 0.00(\mathrm{~s}, 9 \mathrm{H}) ;{ }^{13} \mathrm{C}$ NMR $(75$ $\left.\mathrm{MHz}, \mathrm{CDCl}_{3}\right): \delta-1.9,10.6,18.1,22.7,39.5,43.7,74.4,114.3,125.8,133.9,140.9$; MS (EI) $\mathrm{m} / \mathrm{z} 226\left(\mathrm{M}^{+}\right), 211$, 141, 73; HRMS (EI) $m / z$ calculated for $\mathrm{C}_{13} \mathrm{H}_{26} \mathrm{OSi}$ : 226.1753, found 226.1756. 


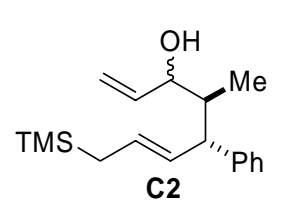

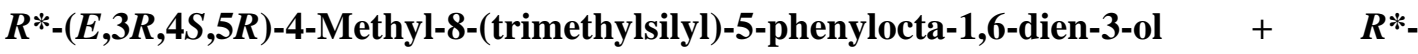
(E,3S,4S,5R)-4-Methyl-8-(trimethylsilyl)-5-phenylocta-1,6-dien-3-ol $\quad(C 2): \quad$ General Procedure $\mathbf{C}$ was followed using $1.00 \mathrm{~g}(3.84 \mathrm{mmol})$ of aldehyde $\mathbf{7 b}($ syn:anti $=92: 8)$ and $5.76 \mathrm{~mL}(5.76 \mathrm{mmol})$ of vinylmagnesium bromide. Purifying the crude product mixture by flash chromatography on $\mathrm{SiO}_{2}(25: 1 \rightarrow 10: 1$ hexanes/EtOAc) yielded $802 \mathrm{mg}(72 \%)$ of the title alcohol as a yellow oil. IR (thin film) 3475, 3083, 3062, 3026, 2954, 1645, 1601, 1248, 966, $854 \mathrm{~cm}^{-1} ;{ }^{1} \mathrm{H}$ $\operatorname{NMR}\left(300 \mathrm{MHz}, \mathrm{CDCl}_{3}\right): \delta 7.34-7.15(\mathrm{~m}, 5 \mathrm{H}), 5.95(\mathrm{ddd}, J=17.2,10.6,4.8 \mathrm{~Hz}, 1 \mathrm{H}), 5.55(\mathrm{dt}, J=15.0,7.4 \mathrm{~Hz}$, $1 \mathrm{H}), 5.46(\mathrm{dd}, J=15.1,8.2 \mathrm{~Hz}, 1 \mathrm{H}), 5.28(\mathrm{dt}, J=17.2,1.6 \mathrm{~Hz}, 1 \mathrm{H}), 5.18(\mathrm{dt}, J=10.6,1.6 \mathrm{~Hz}, 1 \mathrm{H}), 4.55-4.50(\mathrm{~m}$, $1 \mathrm{H}), 3.24(\mathrm{dd}, J=10.2,8.7 \mathrm{~Hz}, 1 \mathrm{H}), 1.91(\mathrm{dqd}, J=10.3,6.9,2.3 \mathrm{~Hz}, 1 \mathrm{H}), 1.46(\mathrm{~d}, J=4.1 \mathrm{~Hz}, 1 \mathrm{H}), 1.43(\mathrm{~d}, J=$ $6.2 \mathrm{~Hz}, 2 \mathrm{H}), 0.66(\mathrm{~d}, J=6.9 \mathrm{~Hz}, 3 \mathrm{H}),-0.05(\mathrm{~s}, 9 \mathrm{H}) ;{ }^{13} \mathrm{C}$ NMR $\left(75 \mathrm{MHz}, \mathrm{CDCl}_{3}\right): \delta-1.8,10.9,22.9,42.9,53.1$, 72.9, 114.1, 125.8, 127.7 (2C), 128.4, 131.6, 140.7, 144.7; MS (EI) m/z 288 (M $\left.{ }^{+}\right), 270,203,73$; HRMS (EI) $\mathrm{m} / \mathrm{z}$ calculated for $\mathrm{C}_{18} \mathrm{H}_{28} \mathrm{OSi}: 288.1909$, found 288.1918 .

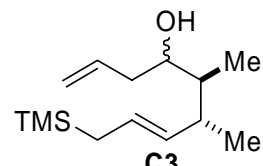

$R^{*}$-(E,4R,5S,6R)-5,6-Dimethyl-9-(trimethylsilyl)nona-1,7-dien-4-ol $+R^{*}-(E, 4 S, 5 S, 6 R)-5,6$ Dimethyl-9-(trimethylsilyl)nona-1,7-dien-4-ol (C3): General Procedure C was followed using $1.9 \mathrm{~g}(9.4 \mathrm{mmol})$ of aldehyde 7a (syn:anti $=93: 7)$ and $11 \mathrm{~mL}(1.2 \mathrm{eq}, 11 \mathrm{mmol})$ of allylmagnesium bromide. Purifying the crude product mixture by flash chromatography on $\mathrm{SiO}_{2}(25: 1$ hexanes/EtOAc) yielded $1.5 \mathrm{~g}(66 \%)$ of the title alcohol as a yellow oil. IR (thin film) 3423, 3077, 2958, 1650, 1641, 1248, $853 \mathrm{~cm}^{-1}$; ${ }^{1} \mathrm{H}$ NMR (300 MHz, $\left.\mathrm{CDCl}_{3}\right): \delta 5.82(\mathrm{ddt}, J=17.1,10.2,7.0 \mathrm{~Hz}, 1 \mathrm{H}), 5.42$ $(\mathrm{dt}, J=15.6,8.0 \mathrm{~Hz}, 1 \mathrm{H}), 5.19(\mathrm{dd}, J=15.2,8.4 \mathrm{~Hz}, 1 \mathrm{H}), 5.15-5.08(\mathrm{~m}, 2 \mathrm{H}), 3.79(\mathrm{ddd}, J=8.2,5.0,3.2 \mathrm{~Hz}, 1 \mathrm{H})$, 2.31-2.08 (m, 3H), $1.50(\mathrm{~s}, 1 \mathrm{H}), 1.43(\mathrm{dd}, J=7.9,0.8 \mathrm{~Hz}, 2 \mathrm{H}), 1.33(\mathrm{pd}, J=6.9,3.1 \mathrm{~Hz}, 1 \mathrm{H}), 0.98(\mathrm{~d}, J=6.8 \mathrm{~Hz}$, $3 \mathrm{H}), 0.90(\mathrm{~d}, J=6.9 \mathrm{~Hz}, 3 \mathrm{H}), 0.00(\mathrm{~s}, 9 \mathrm{H}) ;{ }^{13} \mathrm{C} \mathrm{NMR}\left(75 \mathrm{MHz}, \mathrm{CDCl}_{3}\right): \delta-1.9,10.6,18.5,22.7,39.9,40.0$, 42.9, 71.8, 117.2, 125.7, 133.8, 135.7; MS (EI) $\mathrm{m} / \mathrm{z} 240\left(\mathrm{M}^{+}\right), 225,199,141,73$; HRMS (EI) $\mathrm{m} / \mathrm{z}$ calculated for $\mathrm{C}_{14} \mathrm{H}_{28} \mathrm{OSi}: 240.1909$, found 240.1912 .

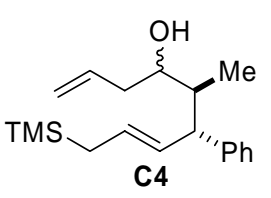

$R^{*}$-(E,4R,5S,6R)-5-Methyl-9-(trimethylsilyl)-6-phenylnona-1,7-dien-4-ol $\quad+\quad R^{*}$ $(E, 4 S, 5 S, 6 R)-5-M e t h y l-9-(t r i m e t h y l s i l y l)-6-p h e n y l n o n a-1,7-d i e n-4-o l \quad$ (C4): General Procedure $\mathbf{C}$ was followed using $918 \mathrm{mg}(3.52 \mathrm{mmol})$ of aldehyde $\mathbf{7 b}($ syn:anti $=92: 8)$ and $5.29 \mathrm{~mL}(5.29 \mathrm{mmol})$ of allylmagnesium bromide. Purifying the crude product mixture by flash chromatography on $\mathrm{SiO}_{2}(25: 1 \rightarrow 10: 1$ hexanes/EtOAc) yielded $0.660 \mathrm{~g}(62 \%)$ of the title alcohol as a yellow oil. IR (thin film) 3475, 3081, 3026, 2953, 1650, 1641, 1601, 1248, 966, $851 \mathrm{~cm}^{-1} ;{ }^{1} \mathrm{H}$ NMR (300 MHz, $\left.\mathrm{CDCl}_{3}\right): \delta$ 7.31-7.14 (m, 5H), $5.85(\mathrm{ddt}, J=17.1,10.1,6.9 \mathrm{~Hz}, 1 \mathrm{H}), 5.52(\mathrm{dt}, J=15.1,7.6 \mathrm{~Hz}$, $1 \mathrm{H}), 5.42(\mathrm{dd}, J=15.1,8.8 \mathrm{~Hz}, 1 \mathrm{H}), 5.15(\mathrm{dq}, J=17.1,1.6 \mathrm{~Hz}, 1 \mathrm{H}), 5.12(\mathrm{dm}, J=10.0 \mathrm{~Hz}, 1 \mathrm{H}), 4.02(\mathrm{dtd}, J=$ 9.2, 4.7, $2.0 \mathrm{~Hz}, 1 \mathrm{H}), 3.21(\mathrm{dd}, J=10.3,9.0 \mathrm{~Hz}, 1 \mathrm{H}), 2.38-2.16(\mathrm{~m}, 2 \mathrm{H}), 1.82(\mathrm{dqd}, J=10.4,6.8,1.8 \mathrm{~Hz}, 1 \mathrm{H})$, 1.42 (br. d, $J=7.2 \mathrm{~Hz}, 2 \mathrm{H}), 1.41$ (d, $J=4.4 \mathrm{~Hz}, 1 \mathrm{H}), 0.69$ (d, $J=6.9 \mathrm{~Hz}, 3 \mathrm{H}),-0.05$ (s, 9H); ${ }^{13} \mathrm{C} \mathrm{NMR}(75 \mathrm{MHz}$, $\left.\mathrm{CDCl}_{3}\right): \delta-1.9,10.5,22.8,40.1,42.0,53.6,70.7,117.5,125.8,127.5,127.7,128.4,131.5,135.6,145.0$; MS (EI) $\mathrm{m} / \mathrm{z} 302\left(\mathrm{M}^{+}\right), 261,245,203,73$; HRMS (EI) $\mathrm{m} / \mathrm{z}$ calculated for $\mathrm{C}_{19} \mathrm{H}_{30} \mathrm{OSi}$ : 302.2066, found 302.2081.

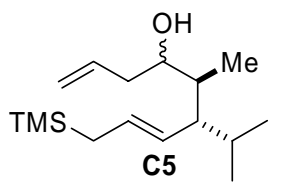

$R^{*}$-(E,4R,5S,6R)-6-Isopropyl-5-methyl-9-(trimethylsilyl)nona-1,7-dien-4-ol $\quad+\quad R^{*}$ (E,4S,5S,6R)-6-Isopropyl-5-methyl-9-(trimethylsilyl)nona-1,7-dien-4-ol (C5): General Procedure C was followed using $1.07 \mathrm{~g}(4.70 \mathrm{mmol})$ of aldehyde 7c (syn:anti: $Z_{\text {syn }}=$ $81: 7: 12)$ and $7.06 \mathrm{~mL}(7.06 \mathrm{mmol})$ of allylmagnesium bromide. Purifying the crude product mixture by flash chromatography on $\mathrm{SiO}_{2}(25: 1$ hexanes/EtOAc) yielded $853 \mathrm{mg}(68 \%)$ of the title alcohol as a yellow oil. IR (thin film) 3439, 3076, 2957, 1646, 1641, 1248, 974, $854 \mathrm{~cm}^{-1} ;{ }^{1} \mathrm{H}$ NMR $\left(300 \mathrm{MHz}, \mathrm{CDCl}_{3}\right): \delta$ 5.81 (ddt, $J=17.2,10.1,7.1 \mathrm{~Hz}, 1 \mathrm{H}), 5.40(\mathrm{dt}, J=15.5,7.9 \mathrm{~Hz}, 1 \mathrm{H}), 5.13-5.01(\mathrm{~m}, 3 \mathrm{H}), 3.83(\mathrm{dtd}, J=9.3,4.9$, $1.7 \mathrm{~Hz}, 1 \mathrm{H}), 2.35-2.24(\mathrm{~m}, 1 \mathrm{H}), 2.16-2.07(\mathrm{~m}, 1 \mathrm{H}), 1.95-1.71(\mathrm{~m}, 2 \mathrm{H}), 1.55-1.46(\mathrm{~m}, 1 \mathrm{H}), 1.48$ (br. d, $J=8.4 \mathrm{~Hz}$, 2H), 1.45 (br. d, $J=4.4 \mathrm{~Hz}, 1 \mathrm{H}), 0.87$ (d, $J=5.9 \mathrm{~Hz}, 3 \mathrm{H}), 0.85$ (d, $J=5.7 \mathrm{~Hz}, 3 \mathrm{H}), 0.75(\mathrm{~d}, J=6.7 \mathrm{~Hz}, 3 \mathrm{H}), 0.01$ $(\mathrm{s}, 9 \mathrm{H}) ;{ }^{13} \mathrm{C} \mathrm{NMR}\left(75 \mathrm{MHz}, \mathrm{CDCl}_{3}\right): \delta-2.1,10.5,16.5,22.2,23.1,27.7,38.5,40.0,52.2,71.4,116.9,127.8$, 
129.3, 136.0; MS (EI) m/z $268\left(\mathrm{M}^{+}\right), 253,225,211,167,73$; HRMS (EI) $\mathrm{m} / \mathrm{z}$ calculated for $\mathrm{C}_{16} \mathrm{H}_{32} \mathrm{OSi}$ : 268.2222, found 268.2218 .

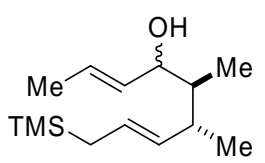

$R^{*}$-(2E,4R,5S,6R,7E)-5,6-Dimethyl-9-(trimethylsilyl)nona-2,7-dien-4-ol

$+\quad R^{*}$ (2E,4S,5S,6R,7E)-5,6-Dimethyl-9-(trimethylsilyl)-nona-2,7-dien-4-ol (10a): The $\operatorname{Ir}(\mathrm{PCy})_{3}{ }^{+}$ isomerization catalyst was prepared according to General Procedure B using $25 \mathrm{mg}(0.028$ $\mathrm{mmol})$ of $\left[\mathrm{IrCl}\left(\mathrm{C}_{8} \mathrm{H}_{14}\right)_{2}\right]_{2}, 19 \mathrm{mg}(0.056 \mathrm{mmol})$ of $\mathrm{NaBPh}_{4}$ and $47 \mathrm{mg}(0.17 \mathrm{mmol})$ of $\mathrm{PCy}_{3}$. To this catalyst solution was added a solution of $1.34 \mathrm{~g}(5.57 \mathrm{mmol})$ of the homoallylic alcohol in $1.5 \mathrm{ml}$ of $\mathrm{CH}_{2} \mathrm{Cl}_{2}$ via cannula. The flask was rinsed with an additional $1.5 \mathrm{~mL}$ of $\mathrm{CH}_{2} \mathrm{Cl}_{2}$ and this rinse was added to the catalyst solution. After $12 \mathrm{~h}$, the reaction was diluted with hexanes and the heterogeneous mixture was filtered through florsil. The crude product mixture was purified by flash chromatography on $\mathrm{SiO}_{2}(25: 1$ hexanes/EtOAc) to afford $1.21 \mathrm{~g}(90 \%)$ of the title compound mixture as a clear oil. The high $\mathrm{R}_{\mathrm{f}}$ diastereomer was isolated by flash chromatography and fully characterized: IR (thin film) 3385, 2958, 1671, 1652, 1248, 967, $851 \mathrm{~cm}^{-1} ;{ }^{1} \mathrm{H}$ NMR (300 MHz, $\left.\mathrm{CDCl}_{3}\right): \delta 5.65(\mathrm{dqd}, J=15.3,7.5,1.0 \mathrm{~Hz}, 1 \mathrm{H}), 5.51(\mathrm{ddq}, J=15.3,6.4,1.4 \mathrm{~Hz}, 1 \mathrm{H}), 5.41$ (dtd, $J=15.2,7.7,0.7 \mathrm{~Hz}, 1 \mathrm{H}), 5.21(\mathrm{ddt}, J=15.2,8.0,1.0 \mathrm{~Hz}, 1 \mathrm{H}), 4.15-4.10(\mathrm{~m}, 1 \mathrm{H}), 2.16$ (sextet, $J=7.0 \mathrm{~Hz}, 1 \mathrm{H})$, $1.72(\mathrm{dt}, J=6.2,1.1 \mathrm{~Hz}, 3 \mathrm{H}), 1.43(\mathrm{dd}, J=7.9,0.7 \mathrm{~Hz}, 2 \mathrm{H}), 1.42-1.34(\mathrm{~m}, 2 \mathrm{H}), 0.94(\mathrm{~d}, J=6.8 \mathrm{~Hz}, 3 \mathrm{H}), 0.88(\mathrm{~d}$, $J=6.9 \mathrm{~Hz}, 3 \mathrm{H}), 0.00(\mathrm{~s}, 9 \mathrm{H}) ;{ }^{13} \mathrm{C} \mathrm{NMR}\left(75 \mathrm{MHz}, \mathrm{CDCl}_{3}\right): \delta-1.9,10.7,17.4,17.7,22.7,39.1,44.1,74.6,125.5$, 126.3, 133.6, 134.0; MS (EI) m/z $240\left(\mathrm{M}^{+}\right), 225,197,141,73$; HRMS (EI) $\mathrm{m} / \mathrm{z}$ calculated for $\mathrm{C}_{14} \mathrm{H}_{28} \mathrm{OSi}$ : 240.1909 , found 240.1913 .

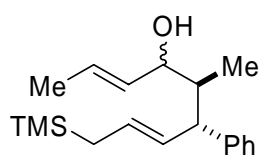

$R^{*}$-(2E,4R,5S,6R,7E)-5-Methyl-9-(trimethylsilyl)-6-phenylnona-2,7-dien-4-ol $\quad+\quad R^{*}$ (2E,4S,5S,6R,7E)-5-Methyl-9-(trimethylsilyl)-6-phenylnona-2,7-dien-4-ol (10b): The $\operatorname{Ir}(\mathrm{PCy})_{3}{ }^{+}$isomerization catalyst was prepared according to General Procedure B using $14.1 \mathrm{mg}$ $(0.0157 \mathrm{mmol})$ of $\left[\mathrm{IrCl}\left(\mathrm{C}_{8} \mathrm{H}_{14}\right)_{2}\right]_{2}, 10.8 \mathrm{mg}(0.0315 \mathrm{mmol})$ of $\mathrm{NaBPh}_{4}$ and $26.5 \mathrm{mg}(0.0944$ mmol) of $\mathrm{PCy}_{3}$ in $1.6 \mathrm{~mL}$ of $50: 1 \mathrm{CH}_{2} \mathrm{Cl}_{2}$ :acetone. This solution of the active catalyst was added to a solution of $476 \mathrm{mg}(1.57 \mathrm{mmol})$ of the homoallylic alcohol in $1.6 \mathrm{~mL}$ of $50: 1 \mathrm{CH}_{2} \mathrm{Cl}_{2}$ :acetone. After $12 \mathrm{~h}$, the reaction was diluted with hexanes and the heterogeneous mixture was filtered through florsil. The crude product mixture was purified by flash chromatography on $\mathrm{SiO}_{2}(15: 1$ hexanes/EtOAc) yielding $384 \mathrm{mg}(81 \%)$ of the product as an oil. The high $\mathrm{R}_{\mathrm{f}}$ diastereomer was isolated via flash chromatography and fully characterized: IR (thin film) 3469, $3061,3026,2954,1653,1601,1248,964,854 \mathrm{~cm}^{-1}$; ${ }^{1} \mathrm{H}$ NMR $\left(300 \mathrm{MHz}, \mathrm{CDCl}_{3}\right): \delta 7.34-7.14(\mathrm{~m}, 5 \mathrm{H}), 5.70(\mathrm{dqd}$, $J=15.3,6.2,1.0 \mathrm{~Hz}, 1 \mathrm{H}), 5.63-5.56(\mathrm{~m}, 1 \mathrm{H}), 5.53(\mathrm{dd}, J=12.7,5.4 \mathrm{~Hz}, 1 \mathrm{H}), 5.44(\mathrm{dd}, J=15.1,8.2 \mathrm{~Hz}, 1 \mathrm{H})$, 4.44-4.37 (m, 1H), $3.23(\mathrm{dd}, J=10.2,8.5 \mathrm{~Hz}, 1 \mathrm{H}), 1.87(\mathrm{dqd}, J=10.3,6.9,2.5 \mathrm{~Hz}, 1 \mathrm{H}), 1.74(\mathrm{~d}, J=6.0 \mathrm{~Hz}, 3 \mathrm{H})$, 1.43 (br. d, $J=7.5 \mathrm{~Hz}, 2 \mathrm{H}), 1.39$ (d, $J=5.4 \mathrm{~Hz}, 1 \mathrm{H}), 0.68$ (d, $J=6.9 \mathrm{~Hz}, 3 \mathrm{H}),-0.04(\mathrm{~s}, 9 \mathrm{H}) ;{ }^{13} \mathrm{C} \mathrm{NMR}(75 \mathrm{MHz}$, $\left.\mathrm{CDCl}_{3}\right): \delta-1.9,11.1,17.8,22.8,43.2,53.1,72.9,125.8,126.2,127.6,127.7,128.4,131.6,133.2,144.8$; MS (EI) $\mathrm{m} / \mathrm{z} 302\left(\mathrm{M}^{+}\right), 287,203,73$; HRMS (EI) $\mathrm{m} / \mathrm{z}$ calculated for $\mathrm{C}_{19} \mathrm{H}_{30} \mathrm{OSi}$ : 302.2066, found 302.2057.

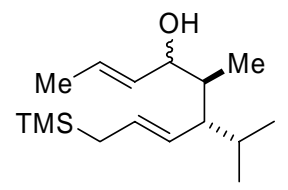

$R^{*}$-(2E,4R,5S,6R,7E)-6-Isopropyl-5-methyl-9-(trimethylsilyl)-nona-2,7-dien-4-ol $+R^{*}$ (2E,4S,5S,6R,7E)-6-Isopropyl-5-methyl-9-(trimethylsilyl)nona-2,7-dien-4-ol (10c): The $\operatorname{Ir}(\mathrm{PCy})_{3}{ }^{+}$isomerization catalyst was prepared according to General Procedure $\mathbf{B}$ using 4.1 $\mathrm{mg}(0.0046 \mathrm{mmol})$ of $\left[\mathrm{IrCl}\left(\mathrm{C}_{8} \mathrm{H}_{14}\right)_{2}\right]_{2}, 3.1 \mathrm{mg}(0.0091 \mathrm{mmol})$ of $\mathrm{NaBPh}_{4}$ and $7.6 \mathrm{mg}(0.027$ $\mathrm{mmol})$ of $\mathrm{PCy}_{3}$ in $1.5 \mathrm{~mL}$ of $50: 1$ of $\mathrm{CH}_{2} \mathrm{Cl}_{2}$ :acetone. This catalyst solution was added to $123 \mathrm{mg}(0.458 \mathrm{mmol})$ of the neat homoallylic alcohol. After $12 \mathrm{~h}$, the reaction was diluted with hexanes and the resulting heterogeneous mixture was filtered through florsil. The crude product was purified by flash chromatography on $\mathrm{SiO}_{2}\left(25: 1\right.$ hexanes/EtOAc) yielding $0.100 \mathrm{~g}(81 \%)$ of the product as an oil. The high $\mathrm{R}_{\mathrm{f}}$ diastereomer was isolated via flash chromatography and fully characterized: IR (thin film) $3465,2958,1665,1655,1248,969,851$ $\mathrm{cm}^{-1} ;{ }^{1} \mathrm{H}$ NMR $\left(300 \mathrm{MHz}, \mathrm{CDCl}_{3}\right): \delta 5.64(\mathrm{dqd}, J=15.4,6.2,1.1 \mathrm{~Hz}, 1 \mathrm{H}), 5.54(\mathrm{ddq}, J=15.4,5.5,1.2 \mathrm{~Hz}, 1 \mathrm{H})$, 5.41 (dt, $J=15.2,7.9 \mathrm{~Hz}, 1 \mathrm{H}), 5.09$ (ddt, $J=15.2,9.9,1.0 \mathrm{~Hz}, 1 \mathrm{H}), 4.29-4.26(\mathrm{~m}, 1 \mathrm{H}), 1.93-1.77(\mathrm{~m}, 2 \mathrm{H}), 1.71$ $(\mathrm{dt}, J=6.0,1.1 \mathrm{~Hz}, 3 \mathrm{H}), 1.59-1.47(\mathrm{~m}, 1 \mathrm{H}), 1.49(\mathrm{dt}, J=7.9,1.2 \mathrm{~Hz}, 2 \mathrm{H}), 1.43(\mathrm{~d}, J=5.4 \mathrm{~Hz}, 1 \mathrm{H}), 0.86(\mathrm{~d}, J=$ 
$6.9 \mathrm{~Hz}, 3 \mathrm{H}), 0.85(\mathrm{~d}, J=6.6 \mathrm{~Hz}, 3 \mathrm{H}), 0.76(\mathrm{~d}, J=6.7 \mathrm{~Hz}, 3 \mathrm{H}), 0.02(\mathrm{~s}, 9 \mathrm{H}) ;{ }^{13} \mathrm{C} \mathrm{NMR}\left(75 \mathrm{MHz}, \mathrm{CDCl}_{3}\right): \delta-1.8$, 11.1, 16.3, 17.7, 22.2, 23.1, 27.6, 40.0, 51.7, 73.3, 125.4, 127.9, 129.4, 133.5; MS (EI) m/z 225 (M $\left.{ }^{+}-{ }^{i} \operatorname{Pr}\right), 169$, 149, 73; HRMS (EI) $\mathrm{m} / \mathrm{z}$ calculated for $\mathrm{C}_{13} \mathrm{H}_{25} \mathrm{OSi}\left(\mathrm{M}^{+}{ }^{-} \mathrm{Pr}\right): 225.1675$, found 225.1669 .

General Procedure D for Preparing Unsaturated Ketones 8 and 9: Ketones 8 and 9 were prepared using a modification of the literature procedure. ${ }^{6}$ To a solution of the diastereomeric mixture of alcohols obtained from General Procedure $\mathrm{C}(1.0 \mathrm{mmol})$ in $3.7 \mathrm{~mL}$ of $\mathrm{CH}_{2} \mathrm{Cl}_{2}$ and $2.9 \mathrm{~mL}$ of DMSO was added $0.70 \mathrm{~mL}(5.0 \mathrm{mmol}) \mathrm{of}$ triethylamine. The resulting solution was cooled to $0{ }^{\circ} \mathrm{C}$ and $0.48 \mathrm{~g}(3.0 \mathrm{mmol})$ of pyridine-sulfur trioxide was added in a single portion. The reaction was stirred for $1-4 \mathrm{~h}$ at $0{ }^{\circ} \mathrm{C}$ until complete as monitored by TLC. The reaction was quenched by adding $\mathrm{pH} 7$ buffer and the layers were separated. The aqueous layer was extracted with $\mathrm{Et}_{2} \mathrm{O}(3 \mathrm{x})$, the combined organic extracts were dried $\left(\mathrm{MgSO}_{4}\right)$ and concentrated. The crude product mixture was purified as indicated. The purified ketone products contained the desired $\mathrm{C}_{5,6}$ syn compound in addition to the corresponding ketones derived from the anti Claisen diastereomer and the $Z$ allyl silane isomer. Purified product composition was established by means of GC-MS [HP-1 (12 m x $0.20 \mathrm{~mm})$, pressure $21 \mathrm{kPa}$, method: $70^{\circ} \mathrm{C}$ for $2.00 \mathrm{~min}$, ramp @ $10^{\circ} \mathrm{C} / \mathrm{min}$ to $300^{\circ} \mathrm{C}$, hold for $60 \mathrm{~min}$ ] and $/$ or ${ }^{1} \mathrm{H}-\mathrm{NMR}$.

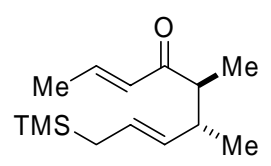

$R^{*}$-(2E,5S,6R,7E)-5,6-Dimethyl-9-(trimethylsilyl)nona-2,7-dien-4-one $\quad(9 a): \quad$ General Procedure D was followed using $1.38 \mathrm{~g}(5.74 \mathrm{mmol})$ of allylic alcohol 10a (mixture of diastereomers). Purifying the crude product by flash chromatography on $\mathrm{SiO}_{2}(25: 1$ hexanes/EtOAc) afforded $1.18 \mathrm{~g}(86 \%)$ of the product as a clear oil. Separation of the diastereomers by GC-MS provided the diastereomer ratio: $7.9 \%$ (anti diastereomer $\mathrm{T}_{\mathrm{r}}=10.35$ ), $82.5 \%$ (syn diastereomer $\left.\mathrm{T}_{\mathrm{r}}=10.49\right), 9.7 \%\left(\mathrm{C}_{7,8} \mathrm{Z} \mathrm{T} \mathrm{T}_{\mathrm{r}}=11.69\right)$. Product ratio by ${ }^{1} \mathrm{H}-\mathrm{NMR}\left(300 \mathrm{MHz}, \mathrm{Si}\left(\mathrm{C}_{3}\right)_{3}\right): 8 \%(Z$ allyl silane $\delta$ 0.011), $7 \%$ (anti diastereomer $\delta-0.005$ ), $85 \%$ (syn diastereomer $\delta-0.029$ ). IR (thin film) 2959, 1696, $1670,1631,1248,969,853 \mathrm{~cm}^{-1} ;{ }^{1} \mathrm{H}$ NMR $\left(300 \mathrm{MHz}, \mathrm{CDCl}_{3}\right): \delta 6.90(\mathrm{dq}, J=15.6,6.9 \mathrm{~Hz}, 1 \mathrm{H}), 6.18(\mathrm{dq}, J=$ 15.5, 1.6 Hz, 1H), 5.37 (dtd, $J=15.2,8.0,6.9 \mathrm{~Hz}, 1 \mathrm{H}), 5.16(\mathrm{ddt}, J=15.2,7.6,1.0 \mathrm{~Hz}, 1 \mathrm{H}), 2.65(\mathrm{p}, J=6.9 \mathrm{~Hz}$, $1 \mathrm{H}), 2.56-2.36(\mathrm{~m}, 1 \mathrm{H}), 1.89$ (dd, $J=6.9,1.6 \mathrm{~Hz}, 3 \mathrm{H}), 1.38$ (br. dd, $J=7.9,0.8 \mathrm{~Hz}, 2 \mathrm{H}), 1.02(\mathrm{~d}, J=6.9 \mathrm{~Hz}, 3 \mathrm{H})$, $0.93(\mathrm{~d}, J=6.8 \mathrm{~Hz}, 3 \mathrm{H}),-0.03(\mathrm{~s}, 9 \mathrm{H}) ;{ }^{13} \mathrm{C} \mathrm{NMR}\left(75 \mathrm{MHz}, \mathrm{CDCl}_{3}\right): \delta-2.1,12.8,17.2,18.0,22.6,38.9,49.0$, 125.9, 131.1, 132.2, 141.5, 202.9; MS (EI) $\mathrm{m} / \mathrm{z} 239\left(\mathrm{M}^{+}+\mathrm{H}\right), 170,155,141,73,69$; HRMS (EI) $\mathrm{m} / \mathrm{z}$ calculated for $\mathrm{C}_{14} \mathrm{H}_{26} \mathrm{OSi}: 238.1753$, found 238.1758 .

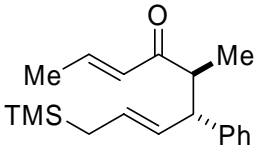

$R^{*}$-(2E,5S,6R,7E)-5-Methyl-9-(trimethylsilyl)-6-phenylnona-2,7-dien-4-one (9b): General Procedure D was followed employing $272 \mathrm{mg}(0.899 \mathrm{mmol})$ of allylic alcohol 10b (mixture of diastereomers). Purifying the crude product by flash chromatography on $\mathrm{SiO}_{2}(20: 1$ hexanes/EtOAc) afforded $167 \mathrm{mg}(62 \%)$ of the product as a clear oil. Product ratio by ${ }^{1} \mathrm{H}-$ NMR $\left(500 \mathrm{MHz}, \mathrm{Si}\left(\mathrm{C}_{3}\right)_{3}\right): 8 \%$ ( $Z$ allyl silane $\delta-0.026$ ), $86 \%$ (syn diastereomer $\delta-0.085$ ), $6 \%$ (anti diastereomer $\delta$-0.114). IR (thin film) 3027, 2954, 1694, 1668, 1629, 1247, $851 \mathrm{~cm}^{-1}$; ${ }^{1} \mathrm{H}$ NMR $(300 \mathrm{MHz}$, $\left.\mathrm{CDCl}_{3}\right): \delta 7.33-7.17(\mathrm{~m}, 5 \mathrm{H}), 6.91(\mathrm{dq}, J=15.6,6.9 \mathrm{~Hz}, 1 \mathrm{H}), 6.21(\mathrm{dq}, J=15.5,1.4 \mathrm{~Hz}, 1 \mathrm{H}), 5.38-5.34(\mathrm{~m}, 2 \mathrm{H})$, 3.50 (dtd, $J=10.4,6.4,3.2 \mathrm{~Hz}, 1 \mathrm{H}), 3.14(\mathrm{dq}, J=10.4,6.9 \mathrm{~Hz}, 1 \mathrm{H}), 1.92(\mathrm{dd}, J=6.8,1.4 \mathrm{~Hz}, 3 \mathrm{H}), 1.35-1.33(\mathrm{~m}$, $2 \mathrm{H}), 0.92(\mathrm{~d}, J=6.9 \mathrm{~Hz}, 3 \mathrm{H}),-0.09(\mathrm{~s}, 9 \mathrm{H}) ;{ }^{13} \mathrm{C} \mathrm{NMR}\left(75 \mathrm{MHz}, \mathrm{CDCl}_{3}\right): \delta-1.9,16.2,18.2,22.8,48.9,52.1$, 126.2, 127.9, 128.1, 128.4, 129.9, 131.3, 142.5, 143.2, 203.1; MS (EI) m/z $301\left(\mathrm{M}^{+}+\mathrm{H}\right), 286,232,203,73$; HRMS (EI) $\mathrm{m} / \mathrm{z}$ calculated for $\mathrm{C}_{19} \mathrm{H}_{28} \mathrm{OSi}$ : 300.1909 , found 300.1921 .

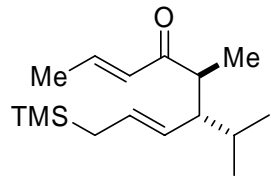

$R^{*}$-(2E,5S,6R,7E)-6-Isopropyl-5-methyl-9-(trimethylsilyl)nona-2,7-dien-4-one

(9c):

General Procedure D was followed employing $1.08 \mathrm{~g}(4.02 \mathrm{mmol})$ of epimeric allylic alcohol 10c (mixture of diastereomers). Purifying the crude product mixture by flash chromatography on $\mathrm{SiO}_{2}(40: 1$ hexanes/EtOAc) afforded $893 \mathrm{mg}(83 \%)$ of the product as a

\footnotetext{
${ }^{6}$ Parikh, J. R.; Doering, W. E. J. Am. Chem. Soc. 1967, 89, 5505.
} 
clear oil. Separation of the diastereomers by GC-MS provided the diastereomer ratio: $81.6 \%$ (syn diastereomer $\left.\mathrm{T}_{\mathrm{r}}=12.02\right), 6.0 \%$ (anti diastereomer $\left.\mathrm{T}_{\mathrm{r}}=12.10\right), 10.6 \%\left(\right.$ syn $\left._{\mathrm{C} 5-6} Z_{\mathrm{C7}-8} \mathrm{~T}_{\mathrm{r}}=12.23\right), 2.0 \%\left(\right.$ anti $_{\mathrm{C} 5-6} Z_{\mathrm{C7}-8} \mathrm{~T}_{\mathrm{r}}=$ 12.34). Product ratio by ${ }^{1} \mathrm{H}-\mathrm{NMR}\left(500 \mathrm{MHz}, \mathrm{Si}\left(\mathrm{C}_{3}\right)_{3}\right): 2 \%$ (anti-Z-diastereomer $\left.\delta=0.020\right), 8 \%$ (Z-allyl silane $\delta 0.004$ ), $5 \%$ (anti-diastereomer $\delta-0.001$ ), $85 \%$ (syn-diastereomer $\delta-0.035$ ). IR (thin film) 2958, 1696, 1671, 1630, 1247, 969, $853 \mathrm{~cm}^{-1}$; ${ }^{1} \mathrm{H}$ NMR $\left(300 \mathrm{MHz}, \mathrm{CDCl}_{3}\right): \delta 6.82(\mathrm{dq}, J=15.4,6.9 \mathrm{~Hz}, 1 \mathrm{H}), 6.15(\mathrm{dq}, J=15.5,1.6$ $\mathrm{Hz}, 1 \mathrm{H}), 5.29(\mathrm{dt}, J=15.3,7.9 \mathrm{~Hz}, 1 \mathrm{H}), 5.00(\mathrm{ddt}, J=15.1,9.6,1.2 \mathrm{~Hz}, 1 \mathrm{H}), 2.81(\mathrm{dq}, J=9.3,6.9 \mathrm{~Hz}, 1 \mathrm{H}), 2.05$ $(\mathrm{dt}, J=9.4,4.3 \mathrm{~Hz}, 1 \mathrm{H}), 1.88(\mathrm{dd}, J=6.9,1.6 \mathrm{~Hz}, 3 \mathrm{H}), 1.90-1.80(\mathrm{~m}, 1 \mathrm{H}), 1.38(\mathrm{dd}, J=7.9,1.2 \mathrm{~Hz}, 2 \mathrm{H}), 1.05(\mathrm{~d}$, $J=6.9 \mathrm{~Hz}, 3 \mathrm{H}), 0.87(\mathrm{~d}, J=6.8 \mathrm{~Hz}, 3 \mathrm{H}), 0.79(\mathrm{~d}, J=6.8 \mathrm{~Hz}, 3 \mathrm{H}),-0.04(\mathrm{~s}, 9 \mathrm{H}) ;{ }^{13} \mathrm{C}$ NMR $\left(75 \mathrm{MHz}, \mathrm{CDCl}_{3}\right)$ : $\delta-6.6,11.6,13.1,14.7,18.5,19.7,24.4,44.3,50.1,129.0,132.9,134.2,145.5,211.1 ; \mathrm{MS}(\mathrm{EI}) \mathrm{m} / \mathrm{z} 266\left(\mathrm{M}^{+}\right)$, 251, 223, 197, 73, 69; HRMS (EI) m/z calculated for $\mathrm{C}_{16} \mathrm{H}_{30} \mathrm{OSi}$ : 266.2066, found 266.2065.

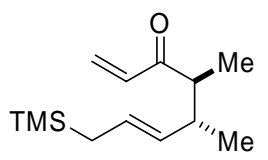

$R^{*}$-(E,4S,5R)-4,5-Dimethyl-8-(trimethylsilyl)octa-1,6-dien-3-one (8a): General Procedure D was followed employing $792 \mathrm{mg}(3.50 \mathrm{mmol})$ of the allylic alcohol (mixture of diastereomers). Purifying the crude product mixture by flash chromatography on $\mathrm{SiO}_{2}(25: 1$ hexanes/EtOAc) afforded $665 \mathrm{mg}(85 \%)$ of the product as a clear oil. Separation of the diastereomers by GCMS provided the diastereomer ratio: $10.2 \%$ (anti diastereomer $\left.\mathrm{T}_{\mathrm{r}}=8.72\right), 77.4 \%$ (syn diastereomer $\mathrm{T}_{\mathrm{r}}=8.90$ ), $12.3 \%\left(\right.$ syn $\left._{\mathrm{C} 4-5} Z_{\mathrm{C} 6-7} \mathrm{~T}_{\mathrm{r}}=8.98\right)$. Product ratio by ${ }^{1} \mathrm{H}-\mathrm{NMR}\left(300 \mathrm{MHz}, \mathrm{Si}\left(\mathrm{C}_{\mathbf{H}}\right)_{3}\right): 9 \%$ (anti diastereomer $\delta 0.011), 10 \%$ ( $Z$ allyl silane $\delta-0.005$ ), $81 \%$ (syn diastereomer $\delta-0.029$ ). IR (thin film) 2958, 1698, 1678, 1612, 1248, 966, $855 \mathrm{~cm}^{-1}$; ${ }^{1} \mathrm{H}$ NMR $\left(300 \mathrm{MHz}, \mathrm{CDCl}_{3}\right): \delta 6.43(\mathrm{dd}, J=17.4,10.4 \mathrm{~Hz}, 1 \mathrm{H}), 6.23(\mathrm{dd}, J=17.4$, $1.6 \mathrm{~Hz}, 1 \mathrm{H}), 5.73(\mathrm{dd}, J=10.4,1.6 \mathrm{~Hz}, 1 \mathrm{H}), 5.38(\mathrm{dtd}, J=15.2,8.0,1.0 \mathrm{~Hz}, 1 \mathrm{H}), 5.16(\mathrm{ddt}, J=15.2,7.6,1.1 \mathrm{~Hz}$, $1 \mathrm{H}), 2.74$ (p, $J=6.9 \mathrm{~Hz}, 1 \mathrm{H}), 2.54-2.43(\mathrm{~m}, 1 \mathrm{H}), 1.38$ (br. dd, $J=8.0,0.9 \mathrm{~Hz}, 2 \mathrm{H}), 1.05(\mathrm{~d}, J=6.9 \mathrm{~Hz}, 3 \mathrm{H}), 0.94$ $(\mathrm{d}, J=6.9 \mathrm{~Hz}, 3 \mathrm{H}),-0.03(\mathrm{~s}, 9 \mathrm{H}) ;{ }^{13} \mathrm{C} \mathrm{NMR}\left(75 \mathrm{MHz}, \mathrm{CDCl}_{3}\right): \delta-2.1,12.7,17.2,22.5,38.9,49.1,126.1,127.5$, 131.9, 135.6, 203.2; MS (EI) m/z $224\left(\mathrm{M}^{+}\right), 209,141$, 73; HRMS (EI) $\mathrm{m} / \mathrm{z}$ calculated for $\mathrm{C}_{13} \mathrm{H}_{24} \mathrm{OSi}$ : 224.1596, found 224.1599 .

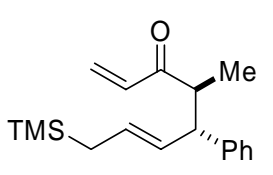

$R^{*}$-(E,4S,5R)-4-Methyl-8-(trimethylsilyl)-5-phenylocta-1,6-dien-3-one (8b): Dess-Martin reagent was prepared and used according to literature precedent. ${ }^{7,8}$ To $0{ }^{\circ} \mathrm{C}$ a solution of 813 $\mathrm{mg}(2.82 \mathrm{mmol})$ of the allylic alcohol (mixture of diastereomers) in $30 \mathrm{~mL}$ of $\mathrm{CH}_{2} \mathrm{Cl}_{2}$ was added $1.80 \mathrm{~g}(4.23 \mathrm{mmol})$ of freshly-prepared Dess-Martin periodinane. The reaction was stirred at $0^{\circ} \mathrm{C}$ for $1 \mathrm{~h}$ then warmed to ambient temperature over an additional $1.5 \mathrm{~h}$. The reaction was diluted with hexanes, filtered through florsil (5:1 hexanes/EtOAc), and the crude product mixture was concentrated. Flash chromatography on $\mathrm{SiO}_{2}(25: 1$ hexanes/EtOAc) yielded $513 \mathrm{mg}(63 \%)$ of the title compound as a clear oil. Separation of the diastereomers by GC-MS provided the diastereomer ratio: $8.1 \%$ (anti diastereomer $\mathrm{T}_{\mathrm{r}}=14.15$ ), $83.8 \%$ (syn diastereomer $\left.\mathrm{T}_{\mathrm{r}}=14.30\right), 8.1 \%\left(\right.$ syn $\left._{\mathrm{C} 4-5} Z_{\mathrm{C} 6-7} \mathrm{~T}_{\mathrm{r}}=14.36\right)$. Product ratio by ${ }^{1} \mathrm{H}-\mathrm{NMR}(300 \mathrm{MHz}$, $\left.\mathrm{Si}\left(\mathrm{C}_{3}\right)_{3}\right): 7 \%$ ( $Z$ allyl silane $\left.\delta-0.035\right), 87 \%$ (syn diastereomer $\left.\delta-0.86\right), 6 \%$ (anti diastereomer $\delta-0.112$ ). IR (thin film) $3061,3026,2954,1698,1678,1611,1248,964,854 \mathrm{~cm}^{-1} ;{ }^{1} \mathrm{H}$ NMR $\left(300 \mathrm{MHz}, \mathrm{CDCl}_{3}\right): \delta 7.34-7.18$ $(\mathrm{m}, 5 \mathrm{H}), 6.46(\mathrm{dd}, J=17.5,10.3 \mathrm{~Hz}, 1 \mathrm{H}), 6.29(\mathrm{dd}, J=17.5,1.4 \mathrm{~Hz}, 1 \mathrm{H}), 5.80(\mathrm{dd}, J=10.3,1.4 \mathrm{~Hz}, 1 \mathrm{H}), 5.39$ $5.36(\mathrm{~m}, 2 \mathrm{H}), 3.52(\mathrm{ddd}, J=10.4,4.1,2.7 \mathrm{~Hz}, 1 \mathrm{H}), 3.21(\mathrm{dq}, J=10.4,6.9 \mathrm{~Hz}, 1 \mathrm{H}), 1.35-1.33(\mathrm{~m}, 2 \mathrm{H}), 0.88(\mathrm{~d}, J$ $=6.9 \mathrm{~Hz}, 3 \mathrm{H}),-0.09(\mathrm{~s}, 9 \mathrm{H}) ;{ }^{13} \mathrm{C} \mathrm{NMR}\left(75 \mathrm{MHz}, \mathrm{CDCl}_{3}\right): \delta-1.9,16.1,22.8,48.5,52.0,126.3,127.9,128.1(2 \mathrm{C})$, 128.5, 129.8, 135.9, 143.0, 203.4; MS (EI) $\mathrm{m} / \mathrm{z} 286\left(\mathrm{M}^{+}\right), 271,257,203,73$; HRMS (EI) $\mathrm{m} / \mathrm{z}$ calculated for $\mathrm{C}_{18} \mathrm{H}_{26} \mathrm{OSi}$ : 286.1753, found 286.1755 .

General Procedure E for Sakurai Annulation Reactions: ${ }^{9}$ To $1.2 \mathrm{~mL}(1.2 \mathrm{mmol})$ of a vigorously stirred solution of $\mathrm{TiCl}_{4}$ in $\mathrm{CH}_{2} \mathrm{Cl}_{2}(1.0 \mathrm{M})$ at $-78{ }^{\circ} \mathrm{C}$ was slowly added the unsaturated ketone 8 or 9 (1.0 mmol) in 10

\footnotetext{
7 Boeckman Jr., R. K.; Shao, P.; Mullins, J. J. Org. Synth., Coll. Vol. X 2000, 2377.

${ }^{8}$ Frigerio, M.; Santagostino, M.; Sputore, S. J. Org. Chem. 1999, 64, 4537.

${ }^{9}$ Hosomi, A.; Sakurai, H. Tetrahedron Lett. 1976, 17, 1295.
} 
$\mathrm{mL}$ of $\mathrm{CH}_{2} \mathrm{Cl}_{2}$ affording a deep red reaction mixture. The syringe and receptacle were washed with $1 \mathrm{~mL}$ of additional $\mathrm{CH}_{2} \mathrm{Cl}_{2}$ and this rinse was added to the reaction vessel. After stirring $15 \mathrm{~min}$ at $-78{ }^{\circ} \mathrm{C}$, the reaction was quenched with by carefully adding sat. aq $\mathrm{NH}_{4} \mathrm{Cl}$ and the resulting mixture was slowly warmed to ambient temperature. The aqueous layer was extracted with $\mathrm{CH}_{2} \mathrm{Cl}_{2}(3 \mathrm{x})$ and the combined organic extracts were dried $\left(\mathrm{Na}_{2} \mathrm{SO}_{4}\right)$ and filtered through a fine glass frit with the filter cake being washed with $\mathrm{Et}_{2} \mathrm{O} .{ }^{10}$ Evaporating the solvents provided the crude product mixture that was purified using the indicated technique. Diastereomer ratios for the purified products were determined using GC-MS [HP-1 $(12 \mathrm{~m} \mathrm{x} 0.20 \mathrm{~mm})$, pressure $21 \mathrm{kPa}$, method: 70 ${ }^{\circ} \mathrm{C}$ for $2.00 \mathrm{~min}$, ramp @ $10{ }^{\circ} \mathrm{C} / \mathrm{min}$ to $300{ }^{\circ} \mathrm{C}$, hold for $\left.60 \mathrm{~min}\right]$.

$R^{*-(2 S, 3 R, 4 S, 5 S)-2,3,5-T r i m e t h y l-4-v i n y l c y c l o h e x a n o n e ~(11): ~ G e n e r a l ~ P r o c e d u r e ~ E ~ w a s ~}$ Me followed employing $0.10 \mathrm{~g}(0.42 \mathrm{mmol})$ of enone 9a (82.5:7.9:9.7 syn:anti:Z). Purifying the crude product mixture by flash chromatography on $\mathrm{SiO}_{2}\left(20: 1\right.$ pentane/ $\left.\mathrm{Et}_{2} \mathrm{O}\right)$ afforded $46 \mathrm{mg}(67$ $\%)$ of the product as a colorless volatile oil. ${ }^{11}$ Separation of the diastereomers by GC-MS provided the diastereomer ratio: $91.7 \%$ (trans, trans, cis $\left.\mathrm{T}_{\mathrm{r}}=6.49\right), 8.3 \%\left(\mathrm{~T}_{\mathrm{r}}=6.99\right)$. IR (thin film) 3076, 2969, 1713, 1638, $915 \mathrm{~cm}^{-1} ;{ }^{1} \mathrm{H}$ NMR (300 MHz, $\left.\mathrm{CDCl}_{3}\right): \delta 5.67(\mathrm{dt}, J=18.0,9.3 \mathrm{~Hz}$, $1 \mathrm{H}), 5.11-5.06(\mathrm{~m}, 2 \mathrm{H}), 2.63(\mathrm{dd}, J=13.0,5.0 \mathrm{~Hz}, 1 \mathrm{H}), 2.37-2.18(\mathrm{~m}, 3 \mathrm{H}), 2.02(\mathrm{dqd}, J=12.9,6.5,0.7 \mathrm{~Hz}, 1 \mathrm{H})$, 1.60-1.46 (m, 1H), $1.04(\mathrm{~d}, J=6.5 \mathrm{~Hz}, 3 \mathrm{H}), 0.98(\mathrm{~d}, J=6.5 \mathrm{~Hz}, 3 \mathrm{H}), 0.83(\mathrm{~d}, J=6.9 \mathrm{~Hz} .3 \mathrm{H}) ;{ }^{13} \mathrm{C}$ NMR $(75$ $\left.\mathrm{MHz}, \mathrm{CDCl}_{3}\right): \delta 11.8,13.9,19.2,36.9,38.8,48.6,50.6,52.2,116.2,140.6,212.2 ; \mathrm{MS}(\mathrm{EI}) \mathrm{m} / \mathrm{z} 166\left(\mathrm{M}^{+}\right), 138$, 96, 68; HRMS (EI) $\mathrm{m} / \mathrm{z}$ calculated for $\mathrm{C}_{11} \mathrm{H}_{18} \mathrm{O}: 166.1358$, found 166.1357 .

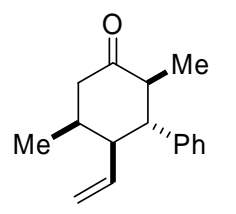

$R^{*}$-(2S,3R,4S,5S)-2,5-Dimethyl-3-phenyl-4-vinylcyclohexanone (14b): General Procedure E was followed employing $0.050 \mathrm{~g}(0.17 \mathrm{mmol})$ of enone $\mathbf{9 b}(86: 6: 8$ syn:anti:Z). Purifying the crude product mixture by flash chromatography on $\mathrm{SiO}_{2}(20: 1$ pentane/Et $2 \mathrm{O})$ afforded $31 \mathrm{mg}(82$ $\%$ ) of the product as a white solid; m.p. $86-88^{\circ} \mathrm{C}$. Separation of the diastereomers by GC-MS provided the diastereomer ratio: $94.6 \%$ (trans, trans, cis $\left.\mathrm{T}_{\mathrm{r}}=12.80\right), 3.8 \%\left(\mathrm{~T}_{\mathrm{r}}=12.99\right), 1.7 \%\left(\mathrm{~T}_{\mathrm{r}}\right.$ $=13.08$ ). IR (thin film) 3064, 3027, 2969, 1712, 1639, $914 \mathrm{~cm}^{-1} ;{ }^{1} \mathrm{H}$ NMR $\left(300 \mathrm{MHz}, \mathrm{CDCl}_{3}\right): \delta 7.34-7.13$ (m, $5 \mathrm{H}), 5.52(\mathrm{ddd}, J=17.8,9.8,8.5 \mathrm{~Hz}, 1 \mathrm{H}), 4.82(\mathrm{dm}, J=10.0 \mathrm{~Hz}, 1 \mathrm{H}), 4.81(\mathrm{dm}, J=17.0 \mathrm{~Hz}, 1 \mathrm{H}), 2.97(\mathrm{td}, J=$ 9.8, 3.3 Hz, 1H), $2.86(\mathrm{dd}, J=13.0,5.4 \mathrm{~Hz}, 1 \mathrm{H}), 2.65(\mathrm{t}, J=11.6 \mathrm{~Hz}, 1 \mathrm{H}), 2.65-2.55(\mathrm{~m}, 1 \mathrm{H}), 2.50-2.45(\mathrm{~m}, 1 \mathrm{H})$, $2.38(\mathrm{dd}, J=12.9,2.9 \mathrm{~Hz}, 1 \mathrm{H}), 0.97(\mathrm{~d}, J=7.1 \mathrm{~Hz}, 3 \mathrm{H}), 0.79(\mathrm{~d}, J=6.1 \mathrm{~Hz}, 3 \mathrm{H}) ;{ }^{13} \mathrm{C}$ NMR $\left(75 \mathrm{MHz}, \mathrm{CDCl}_{3}\right)$ : $\delta 12.2,13.7,37.0,48.8,50.2,50.8,51.7,116.3,126.6,128.0,128.5,139.3,142.4,211.6$; MS (EI) $\mathrm{m} / \mathrm{z} 228\left(\mathrm{M}^{+}\right)$, 118, 68; HRMS (EI) $\mathrm{m} / \mathrm{z}$ calculated for $\mathrm{C}_{16} \mathrm{H}_{20} \mathrm{O}: 228.1514$, found 228.1512 .

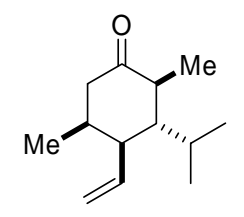

$R^{*}$-(2S,3R,4S,5S)-3-Isopropyl-2,5-dimethyl-4-vinylcyclohexanone

(14c): General Procedure $\mathbf{E}$ was followed employing $0.10 \mathrm{~g}(0.38 \mathrm{mmol})$ of enone 9c (81.4:6.0:10.6:2.0 syn : anti : $\left.\operatorname{syn}_{\mathrm{C} 5-6} Z_{\mathrm{C} 7-8}: a_{\text {anti }} i_{\mathrm{C}-6} Z_{\mathrm{C} 7-8}\right)$. Purifying the crude product mixture by flash chromatography on $\mathrm{SiO}_{2}(25: 1$ hexanes/EtOAc) afforded $65 \mathrm{mg}(87 \%)$ of the product as a clear oil. Separation of the diastereomers by GC-MS provided the diastereomer ratio: $89.0 \%$ (trans, trans, cis $\left.\mathrm{T}_{\mathrm{r}}=8.83\right), 8.2 \%\left(\mathrm{~T}_{\mathrm{r}}=8.99\right), 2.7 \%\left(\mathrm{~T}_{\mathrm{r}}=9.07\right)$. IR (thin film) 3090, 2960, 1713, 1637, $913 \mathrm{~cm}^{-}$ ${ }^{1} ;{ }^{1} \mathrm{H}$ NMR $\left(300 \mathrm{MHz}, \mathrm{CDCl}_{3}\right): \delta 5.65(\mathrm{dt}, J=16.8,10.0,1 \mathrm{H}), 5.04(\mathrm{dd}, J=10.3,1.8 \mathrm{~Hz}, 1 \mathrm{H}), 5.00(\mathrm{dd}, J=16.8$, $1.8 \mathrm{~Hz}, 1 \mathrm{H}), 2.42(\mathrm{dd}, J=16.2,4.8 \mathrm{~Hz}, 1 \mathrm{H}), 2.47-2.36(\mathrm{~m}, 2 \mathrm{H}), 2.34-2.23(\mathrm{~m}, 1 \mathrm{H}), 2.11(\mathrm{~d}, J=16.0,8.9 \mathrm{~Hz}, 1 \mathrm{H})$, 2.04-1.94 (m, 1H), 1.39 (ddd, $J=8.3,5.5,2.8 \mathrm{~Hz}), 1.06(\mathrm{~d}, J=6.4 \mathrm{~Hz}, 3 \mathrm{H}), 0.96$ (d, $J=7.0 \mathrm{~Hz}, 3 \mathrm{H}), 0.95(\mathrm{~d}, J=$ $7.0 \mathrm{~Hz}, 3 \mathrm{H}), 0.90(\mathrm{~d}, J=6.8 \mathrm{~Hz}, 3 \mathrm{H}) ;{ }^{13} \mathrm{C} \mathrm{NMR}\left(75 \mathrm{MHz}, \mathrm{CDCl}_{3}\right): \delta 13.2,16.9,17.5,20.3,29.6,32.7,44.5,45.0$, 45.2, 50.4, 115.7, 140.1, 214.4; MS (EI) $\mathrm{m} / \mathrm{z} 194\left(\mathrm{M}^{+}\right), 179,166,151,68$; HRMS (EI) $\mathrm{m} / \mathrm{z}$ calculated for $\mathrm{C}_{13} \mathrm{H}_{22} \mathrm{O}$ : 194.1671, found 194.1680 .

\footnotetext{
${ }^{10}$ Titanium salts were found to epimerize the $\alpha$-chiral ketone products upon crude product concentration. Filtration through a fine glass frit effectively minimizes these deleterious byproducts.

${ }^{11}$ Due to the volatility of this product, isolated yields were found to improve for larger scale reactions.
} 


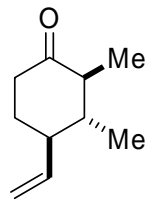

$R^{*}$-(2S,3R,4R)-2,3-Dimethyl-4-vinylcyclohexanone (13a): General Procedure E was followed employing $0.050 \mathrm{~g}(0.22 \mathrm{mmol})$ of acrylate 8a (77.4:10.2:12.3 syn:anti:Z). Purifying the crude product mixture by flash chromatography on $\mathrm{SiO}_{2}(20: 1$ pentane/Et $2 \mathrm{O})$ afforded $0.020 \mathrm{~g}(59 \%)$ of the product as a clear, highly volatile oil. Separation of the diastereomers by GC-MS provided the diastereomer ratio: $89.8 \%$ (trans, trans $\left.\mathrm{T}_{\mathrm{r}}=5.43\right), 10.2 \%\left(\mathrm{~T}_{\mathrm{r}}=5.92\right) . \quad$ IR (thin film) 3077 , 2971, 1713, 1643, $914 \mathrm{~cm}^{-1} ;{ }^{1} \mathrm{H}$ NMR $\left(300 \mathrm{MHz}, \mathrm{CDCl}_{3}\right): \delta 5.57$ (ddd, $J=17.2,10.1,8.8 \mathrm{~Hz}$, $1 \mathrm{H}), 5.06(\mathrm{dd}, J=17.1,2.0 \mathrm{~Hz}, 1 \mathrm{H}), 5.03(\mathrm{dd}, J=10.2,1.8 \mathrm{~Hz}, 1 \mathrm{H}), 2.47-2.33(\mathrm{~m}, 2 \mathrm{H}), 2.17-2.08(\mathrm{~m}, 1 \mathrm{H}), 2.09$ $(\mathrm{dd}, J=11.2,6.7 \mathrm{~Hz}, 1 \mathrm{H}), 2.06-1.95(\mathrm{~m}, 1 \mathrm{H}), 1.67-1.50(\mathrm{~m}, 1 \mathrm{H}), 1.34-1.20(\mathrm{~m}, 1 \mathrm{H}), 1.04(\mathrm{~d}, J=6.5 \mathrm{~Hz}, 3 \mathrm{H})$, $1.01(\mathrm{~d}, J=6.5 \mathrm{~Hz}, 3 \mathrm{H}) ;{ }^{13} \mathrm{C}$ NMR $\left(75 \mathrm{MHz}, \mathrm{CDCl}_{3}\right): \delta 11.8,18.7,33.2,40.9,44.6,48.8,50.4,115.3,141.6$, 212.2; MS (EI) m/z $152\left(\mathrm{M}^{+}\right), 126,111$; HRMS (EI) $\mathrm{m} / \mathrm{z}$ calculated for $\mathrm{C}_{10} \mathrm{H}_{16} \mathrm{O}: 152.1201$, found 152.1196 .

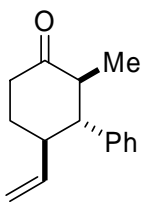

$R^{*}$-(2S,3R,4R)-2-Methyl-3-phenyl-4-vinylcyclohexanone (13b): General Procedure E was followed employing $0.050 \mathrm{~g}(0.17 \mathrm{mmol})$ of acrylate $\mathbf{8 b}$ (83.8:8.1:8.1 syn:anti: $\mathrm{Z})$. Purifying the crude product mixture by flash chromatography on $\mathrm{SiO}_{2}\left(10: 1\right.$ pentane/Et $\left.{ }_{2} \mathrm{O}\right)$ afforded $0.030 \mathrm{~g}(82$ $\%)$ of the product as a clear, viscous oil. Separation of the diastereomers by GC-MS provided the diastereomer ratio: $92.6 \%$ (trans, trans $\left.\mathrm{T}_{\mathrm{r}}=12.10\right), 5.3 \%\left(\mathrm{~T}_{\mathrm{r}}=12.31\right), 2.1 \%\left(\mathrm{~T}_{\mathrm{r}}=12.14\right) . \quad \mathrm{IR}$ (thin film) 3081, 3027, 2970, 1712, $913 \mathrm{~cm}^{-1} ;{ }^{1} \mathrm{H}$ NMR (300 MHz, $\left.\mathrm{CDCl}_{3}\right): \delta 7.34-7.12(\mathrm{~m}, 5 \mathrm{H}), 5.44(\mathrm{ddd}, J=$ 17.6, 10.4, $7.5 \mathrm{~Hz}, 1 \mathrm{H}), 4.81(\mathrm{dm}, J=17.3 \mathrm{~Hz}, 1 \mathrm{H}), 4.78(\mathrm{dm}, J=10.4 \mathrm{~Hz}, 1 \mathrm{H}), 2.82-2.51(\mathrm{~m}, 4 \mathrm{H}), 2.37(\mathrm{t}, J=$ $11.5 \mathrm{~Hz}, 1 \mathrm{H}), 2.20(\mathrm{ddt}, J=13.4,6.1,3.1 \mathrm{~Hz}, 1 \mathrm{H}), 1.75(\mathrm{qd}, J=13.4,5.1 \mathrm{~Hz}, 1 \mathrm{H}), 0.78(\mathrm{~d}, J=6.5 \mathrm{~Hz}, 3 \mathrm{H}) ;{ }^{13} \mathrm{C}$ $\operatorname{NMR}\left(75 \mathrm{MHz}, \mathrm{CDCl}_{3}\right): \delta 12.3,32.5,41.1,47.2,49.9,57.9,114.9,126.6,127.9,128.5,140.1,142.2,211.8$; MS (EI) $\mathrm{m} / \mathrm{z} 214\left(\mathrm{M}^{+}\right), 147,118,68$; HRMS (EI) $\mathrm{m} / \mathrm{z}$ calculated for $\mathrm{C}_{15} \mathrm{H}_{18} \mathrm{O}: 214.1358$, found 214.1353.

General Procedure F for Tandem Intermolecular Sakurai-Aldol Reactions: General Procedure E for the Sakurai annulation reactions was followed as described incoporating the following modifications. After reacting the enone with $\mathrm{TiCl}_{4}$ for the indicated time, neat aldehyde $(1.2 \mathrm{mmol})$ was added dropwise to the deep red solution of the titanium enolate intermediate. The reaction was stirred at $-78{ }^{\circ} \mathrm{C}$ until complete as monitored by TLC $(\sim 1 \mathrm{~h})$. Reaction work-up was performed as described in General Procedure E. Reaction products were purified using the indicated procedures. Diastereomer ratios of the purified products were determined by LC-MS [X-terra C-18, method: 35\%:65\% MeCN: $\mathrm{H}_{2} \mathrm{O}$ for $24.00 \mathrm{~min}$, ramp to 50:50 MeCN: $\mathrm{H}_{2} \mathrm{O}$ ] or $500 \mathrm{MHz}{ }^{1} \mathrm{H}$ NMR data.

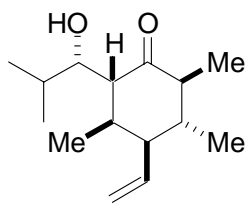

$R^{*}$-(2R,3R,4R,5R,6S)-2-((S)-1-Hydroxy-2-methylpropyl)-3,5,6-trimethyl-4-vinylcyclohexanone (18b): General Procedure $\mathbf{F}$ was followed employing $0.050 \mathrm{~g}(0.21 \mathrm{mmol})$ of enone 9a (82.5:7.9:9.7 syn : anti : Z) and $23 \mu \mathrm{L}(18 \mathrm{mg}, 0.25 \mathrm{mmol})$ of isobutyraldehyde. Flash chromatography on $\mathrm{SiO}_{2}(5: 1$ hexanes/EtOAc) afforded $26 \mathrm{mg}(52 \%)$ of the product as a clear oil. Isolated diastereomer ratio determined by $500 \mathrm{MHz}{ }^{1} \mathrm{H}$ NMR $(\mathbf{C H O H}): 8 \%(\delta 3.98), 85 \%$

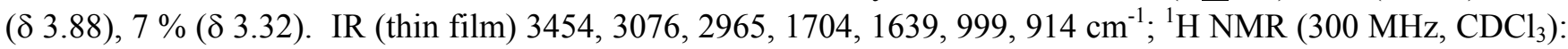
$\delta 5.67(\mathrm{ddd}, J=16.8,10.4,9.0 \mathrm{~Hz}, 1 \mathrm{H}), 5.10(\mathrm{dm}, J=10.3 \mathrm{~Hz}, 1 \mathrm{H}), 5.07(\mathrm{dm}, J=16.8 \mathrm{~Hz}, 1 \mathrm{H}), 3.92-3.86(\mathrm{~m}$, $1 \mathrm{H}), 2.38-2.28(\mathrm{~m}, 3 \mathrm{H}), 2.12(\mathrm{qdd}, J=7.1,4.3,3.1 \mathrm{~Hz}, 1 \mathrm{H}), 1.90(\mathrm{pd}, J=6.9,3.5 \mathrm{~Hz}, 1 \mathrm{H}), 1.63-1.52(\mathrm{~m}, 1 \mathrm{H})$, $1.51(\mathrm{~d}, J=7.8 \mathrm{~Hz}, 1 \mathrm{H}), 1.08(\mathrm{~d}, J=6.5 \mathrm{~Hz}, 3 \mathrm{H}), 1.04(\mathrm{~d}, J=6.9 \mathrm{~Hz}, 3 \mathrm{H}), 1.01(\mathrm{~d}, J=6.4 \mathrm{~Hz}, 3 \mathrm{H}), 0.89(\mathrm{~d}, J=$ $7.2 \mathrm{~Hz}, 3 \mathrm{H}), 0.85(\mathrm{~d}, J=6.8 \mathrm{~Hz}, 3 \mathrm{H}) ;{ }^{13} \mathrm{C} \mathrm{NMR}\left(75 \mathrm{MHz}, \mathrm{CDCl}_{3}\right): \delta 12.4,14.7,15.1,19.2,20.1,30.5,37.9,38.6$, 48.1, 48.4, 61.2, 75.7, 116.4, 140.4, 214.7; MS (EI) m/z $220\left(\mathrm{M}^{+}-\mathrm{H}_{2} \mathrm{O}\right), 205,195,166,98,68$; HRMS (EI) $\mathrm{m} / \mathrm{z}$ calculated for $\mathrm{C}_{15} \mathrm{H}_{24} \mathrm{O}\left(\mathrm{M}^{+}-\mathrm{H}_{2} \mathrm{O}\right): 220.1827$, found 220.1835 .

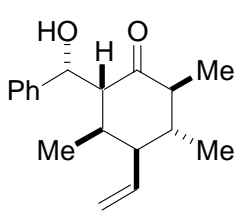

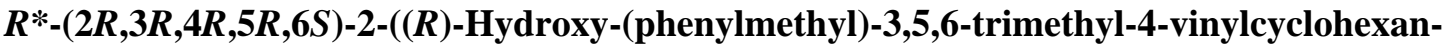
one (18a): General Procedure F was followed employing $0.050 \mathrm{~g}(0.21 \mathrm{mmol})$ of enone 9a (82.5:7.9:9.7 syn : anti : Z) and $25 \mu \mathrm{L}(27 \mathrm{mg}, 0.25 \mathrm{mmol})$ of benzaldehyde. Flash 
chromatography on $\mathrm{SiO}_{2}(5: 1$ hexanes/EtOAc) afforded $31 \mathrm{mg}$ of the product (52\%) as a clear oil. Isolated diastereomer ratio determined by LC-MS [X-terra C-18, method: 35\%:65\% MeCN: $\mathrm{H}_{2} \mathrm{O}$ for 24.00 min, ramp to 50:50 MeCN:H $\left.\mathrm{H}_{2} \mathrm{O}\right]: 3.2 \%\left(\mathrm{~T}_{\mathrm{r}}=34.68\right), 84.9 \%\left(\mathrm{~T}_{\mathrm{r}}=37.49\right), 10.4 \%\left(\mathrm{~T}_{\mathrm{r}}=39.56\right), 1.6 \%\left(\mathrm{~T}_{\mathrm{r}}=42.32\right)$. IR (thin film) 3427, 3067, 3030, 2970, 1698, 1639, $915 \mathrm{~cm}^{-1} ;{ }^{1} \mathrm{H}$ NMR (300 MHz, $\left.\mathrm{CDCl}_{3}\right): \delta$ 7.39-7.24 (m, 5H), 5.67 $(\mathrm{ddd}, J=17.6,9.6,8.7 \mathrm{~Hz}, 1 \mathrm{H}), 5.15-5.06(\mathrm{~m}, 3 \mathrm{H}), 2.61(\mathrm{dd}, J=7.9,3.8 \mathrm{~Hz}, 1 \mathrm{H}), 2.60(\mathrm{~d}, J=3.8 \mathrm{~Hz}, 1 \mathrm{H}), 2.55-$ $2.43(\mathrm{~m}, 2 \mathrm{H}), 1.92(\mathrm{dq}, J=10.6,6.5 \mathrm{~Hz}, 1 \mathrm{H}), 1.53(\mathrm{ddq}, J=10.6,8.9,6.5 \mathrm{~Hz}, 1 \mathrm{H}), 1.00(\mathrm{~d}, J=6.5 \mathrm{~Hz}, 3 \mathrm{H}), 0.92$ $(\mathrm{d}, \quad J=6.4 \mathrm{~Hz}, 3 \mathrm{H}), 0.90(\mathrm{~d}, \quad J=6.9 \mathrm{~Hz}, \quad 3 \mathrm{H}) ;{ }^{13} \mathrm{C} \quad \mathrm{NMR} \quad\left(75 \mathrm{MHz}, \mathrm{CDCl}_{3}\right):$ $\delta 12.2,15.3,19.6,35.6,39.0,48.3,49.8,64.0,73.3,116.4,126.3,128.2,128.6,139.9,141.5,213.9$; MS (EI) $\mathrm{m} / \mathrm{z}$ $272\left(\mathrm{M}^{+}\right), 254,166,106,69$; HRMS (EI) $\mathrm{m} / \mathrm{z}$ calculated for $\mathrm{C}_{18} \mathrm{H}_{24} \mathrm{O}_{2}: 272.1776$, found 272.1784 .

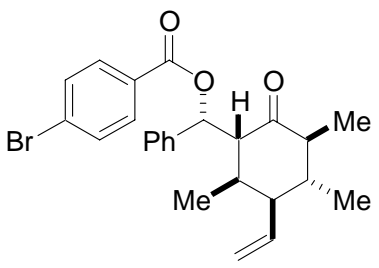

$R *-(R)-((1 R, 2 R, 3 R, 4 R, 5 S)-2,4,5-T r i m e t h y l-6-0 x 0-3-v i n y l c y c l o h e x y l)(p h e n y l)-$ methyl-4-bromobenzoate (19): To $54 \mathrm{mg}(0.20 \mathrm{mmol})$ of cyclohexanone 18a in $2 \mathrm{~mL}$ of $\mathrm{CH}_{2} \mathrm{Cl}_{2}$ at $0{ }^{\circ} \mathrm{C}$ was added $2.4 \mathrm{mg}(0.020 \mathrm{mmol})$ of 4-(dimethylamino)pyridine, 52 $\mathrm{mg}(0.24 \mathrm{mmol})$ of 4-bromobenzoyl chloride, and $42 \mu \mathrm{L}(31 \mathrm{mg}, 0.24 \mathrm{mmol})$ of $N, N$ diisopropylethylamine in succession. The resulting solution was warmed to ambient temperature and stirred for $5 \mathrm{~h}$. The reaction was quenched by adding aq. $1 \mathrm{M} \mathrm{HCl}$ and the aqueous layer was extracted with EtOAc (4x). The combined organic extracts were dried $\left(\mathrm{MgSO}_{4}\right)$, concentrated, and the resulting crude product mixture was purified by flash chromatography on $\mathrm{SiO}_{2}(10: 1$ hexanes/EtOAc) to afford $71 \mathrm{mg}(80 \%)$ of the title compound as a colorless crystalline solid: mp 154$156{ }^{\circ} \mathrm{C}$. Recrystallizing this solid from hexanes/EtOAc provided crystals suitable for X-ray diffraction analysis. IR (thin film) 3069, 2972, 1713, 1591, 1267, $914 \mathrm{~cm}^{-1}$; ${ }^{1} \mathrm{H}$ NMR $\left(300 \mathrm{MHz}, \mathrm{CDCl}_{3}\right): \delta 7.92(\mathrm{~d}, J=8.5 \mathrm{~Hz}, 2 \mathrm{H})$, $7.60(\mathrm{~d}, J=8.5 \mathrm{~Hz}, 2 \mathrm{H}), 7.37-7.31(\mathrm{~m}, 5 \mathrm{H}), 6.56(\mathrm{~d}, J=10.5 \mathrm{~Hz}, 1 \mathrm{H}), 5.64(\mathrm{dt}, J=17.1,10.0 \mathrm{~Hz}, 1 \mathrm{H}), 5.09(\mathrm{~d}, J$ $=17.2 \mathrm{~Hz}, 1 \mathrm{H}), 5.08(\mathrm{~d}, J=10.1 \mathrm{~Hz}, 1 \mathrm{H}), 2.99(\mathrm{dd}, J=10.5,2.3 \mathrm{~Hz}, 1 \mathrm{H}), 2.75(\mathrm{td}, J=10.8,4.1 \mathrm{~Hz}, 1 \mathrm{H}), 2.43-$ $2.28(\mathrm{~m}, 1 \mathrm{H}), 2.16(\mathrm{dq}, J=12.7,6.4 \mathrm{~Hz}, 1 \mathrm{H}), 1.63-1.53(\mathrm{~m}, 1 \mathrm{H}), 1.05(\mathrm{~d}, J=6.4 \mathrm{~Hz}, 3 \mathrm{H}), 0.94(\mathrm{~d}, J=7.7 \mathrm{~Hz}$, $3 \mathrm{H}), 0.92(\mathrm{~d}, J=6.6 \mathrm{~Hz}, 3 \mathrm{H}) ;{ }^{13} \mathrm{C} \mathrm{NMR}\left(75 \mathrm{MHz}, \mathrm{CDCl}_{3}\right): \delta 11.8,14.5,19.2,38.2,39.5,47.3,49.7,64.0,74.6$, 116.9, 127.1, 128.4, 128.7, 128.8, 129.0, 131.2, 131.8, 137.2, 139.8, 165.0, 210.4; MS (EI) m/z $456\left(\mathrm{M}^{+}\right), 271$, 254, 185, 183; HRMS (EI) $\mathrm{m} / \mathrm{z}$ calculated for $\mathrm{C}_{25} \mathrm{H}_{27} \mathrm{O}_{3} \mathrm{Br}$ : 454.1144, found 454.1139.

Pent-4-ynyl benzoate: ${ }^{12}$ To $0{ }^{\circ} \mathrm{C}$ solution of $1.0 \mathrm{~g}(1.1 \mathrm{~mL}, 12 \mathrm{mmol})$ of 4-pentyn-1-ol in 40 BzO $\mathrm{mL}$ of $\mathrm{CH}_{2} \mathrm{Cl}_{2}$ was successively added $2.0 \mathrm{~g}$ (14 mmol) of benzoyl chloride and $145 \mathrm{mg}(1.19$ mmol) of 4-(dimethylamino)pyridine, and $2.5 \mathrm{~mL}(1.8 \mathrm{~g}, 14 \mathrm{mmol})$ of $N, N$-diisopropylethylamine. The reaction solution was warmed to ambient temperature and stirred for $12 \mathrm{~h}$. The reaction mixture was poured into aq. $1 \mathrm{M}$ $\mathrm{HCl}$ and the aqueous layer was extracted with EtOAc $(4 \mathrm{x})$. The combined organic extracts were washed with brine, dried $\left(\mathrm{MgSO}_{4}\right)$, and concentrated. The resulting crude product mixture was purified by flash chromatography on $\mathrm{SiO}_{2}\left(20: 1\right.$ hexanes/EtOAc) to afford $2.0 \mathrm{~g}(90 \%)$ of the product as a clear oil. ${ }^{1} \mathrm{H}$ NMR (300 $\left.\mathrm{MHz}, \mathrm{CDCl}_{3}\right): \delta 8.08-8.04(\mathrm{~m}, 2 \mathrm{H}), 7.60-7.54(\mathrm{~m}, 1 \mathrm{H}), 7.48-7.42(\mathrm{~m}, 2 \mathrm{H}), 4.44(\mathrm{t}, J=6.2 \mathrm{~Hz}, 2 \mathrm{H}), 2.40(\mathrm{td}, J=$ $7.1,2.6 \mathrm{~Hz}, 2 \mathrm{H}), 2.02(\mathrm{q}, J=6.5 \mathrm{~Hz}, 2 \mathrm{H}), 2.01-1.99(\mathrm{~m}, 1 \mathrm{H})$.

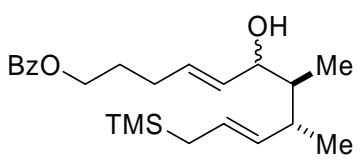

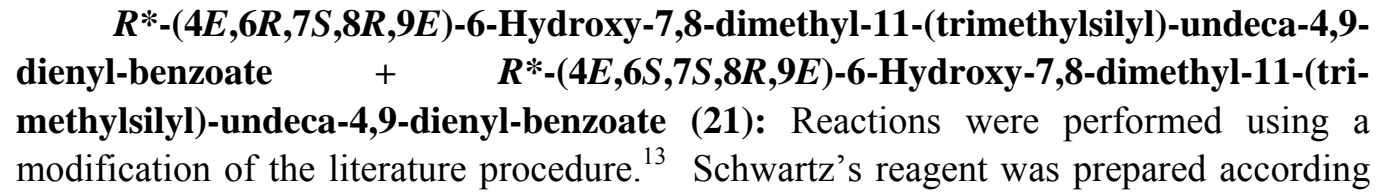
to literature methods. ${ }^{14}$ To $0{ }^{\circ} \mathrm{C}$ solution of $221 \mathrm{mg}(0.861 \mathrm{mmol})$ of di(cyclopentadienyl)zirconium chloride hydride in $2.5 \mathrm{~mL}$ of $\mathrm{CH}_{2} \mathrm{Cl}_{2}$ added $171 \mathrm{mg}(0.909 \mathrm{mmol})$ of pent-4-ynyl benzoate. The reaction mixture was warmed to ambient temperature and stirred until homogenous. To this stirring solution was added $0.100 \mathrm{~g}(0.504$

\footnotetext{
${ }^{12}$ Sato, H.; Isono, N.; Miyoshi, I.; Mori, M. Tetrahedron 1996, 52, 8143.

${ }^{13}$ Suzuki, K.; Hasegawa, T.; Imai, T.; Maeta, H.; Ohba, S. Tetrahedron 1995, 51, 4483.

${ }^{14}$ Buchwald, S. L.; LaMaire, S. J.; Nielsen, R. B.; Watson, B. T.; King, S. M. Org. Synth., Coll. Vol. IX 1993, 162.
} 
mmol) of aldehyde 18 (93:7 syn : anti). A separate flask was charged with $15 \mathrm{mg}(0.050 \mathrm{mmol})$ of $\mathrm{AgAsF}_{6}$ and $2.5 \mathrm{~mL}$ of $\mathrm{CH}_{2} \mathrm{Cl}_{2}$ and the reaction solution prepared above was added carefully to this flask via syringe (colorless-to-brown color change). The resulting brown solution was stirred for $10 \mathrm{~min}$ then the reaction was quenched by adding sat. aq. $\mathrm{NaHCO}_{3}$. The aqueous layer was extracted with EtOAc (4x) the combined organic extracts were dried $\left(\mathrm{Na}_{2} \mathrm{SO}_{4}\right)$ and concentrated. The resulting crude product mixture was purified by flash chromatography on $\mathrm{SiO}_{2}(5: 1$ hexanes/EtOAc) to afford $159 \mathrm{mg}(81 \%)$ of the title compound as a mixture of C-6 epimers The higher $\mathrm{R}_{\mathrm{f}}$ diastereomer was isolated via flash chromatography and fully characterized. IR (thin film) 3515, 2957, 1721, 1602, 1274, 1248, 970, 853, $675 \mathrm{~cm}^{-1} ;{ }^{1} \mathrm{H}$ NMR $\left(300 \mathrm{MHz}, \mathrm{CDCl}_{3}\right): \delta 8.05(\mathrm{dd}, J=7.1$, $1.4 \mathrm{~Hz}, 2 \mathrm{H}), 7.57(\mathrm{tt}, J=7.3,1.3 \mathrm{~Hz}, 1 \mathrm{H}), 7.45(\mathrm{t}, J=7.3 \mathrm{~Hz}, 2 \mathrm{H}), 5.68(\mathrm{dt}, J=15.4,6.5 \mathrm{~Hz}, 1 \mathrm{H}), 5.55(\mathrm{dd}, J=$ $15.4,6.0 \mathrm{~Hz}, 1 \mathrm{H}), 5.41(\mathrm{dt}, J=15.2,7.9 \mathrm{~Hz}, 1 \mathrm{H}), 5.21(\mathrm{dd}, J=15.2,8.1 \mathrm{~Hz}, 1 \mathrm{H}), 4.35(\mathrm{t}, J=6.6 \mathrm{~Hz}, 2 \mathrm{H}), 4.21-$ $4.14(\mathrm{~m}, 1 \mathrm{H}), 2.24(\mathrm{q}, J=6.9 \mathrm{~Hz}, 2 \mathrm{H}), 2.22-2.09(\mathrm{~m}, 1 \mathrm{H}), 1.88(\mathrm{q}, J=6.6 \mathrm{~Hz}, 2 \mathrm{H}), 1.43-1.33(\mathrm{~m}, 1 \mathrm{H}), 1.43$ (br. $\mathrm{d}, J=7.9 \mathrm{~Hz}, 2 \mathrm{H}), 0.95(\mathrm{~d}, J=6.8 \mathrm{~Hz}, 3 \mathrm{H}), 0.88(\mathrm{~d}, J=6.9 \mathrm{~Hz}, 3 \mathrm{H}), 0.00(\mathrm{~s}, 9 \mathrm{H}) ;{ }^{13} \mathrm{C} \mathrm{NMR}\left(75 \mathrm{MHz}, \mathrm{CDCl}_{3}\right)$ : $\delta-1.9,10.7,17.7,22.7,28.4,28.8,39.3,44.1,64.3,74.2,125.6,128.3,129.6,129.8,130.5,132.8,133.4,133.9$, 166.6; MS (ESI) m/z $411\left(\mathrm{M}^{+}+\mathrm{Na}\right)$; HRMS (ESI) $\mathrm{m} / \mathrm{z}$ calculated for $\mathrm{NaC}_{23} \mathrm{H}_{36} \mathrm{O}_{3} \mathrm{Si}\left(\mathrm{M}^{+}+\mathrm{Na}\right)$ : 411.2331, found 411.2340 .

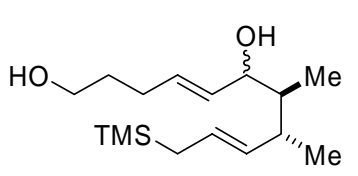

$R^{*}$-(4E,6R,7S,8R,9E)-7,8-Dimethyl-11-(trimethylsilyl)-undeca-4,9-diene-1,6-diol $\quad+$ $R^{*}$-(4E,6S,7S,8R,9E)-7,8-Dimethyl-11-(trimethylsilyl)-undeca-4,9-diene-1,6-diol:

Reactions were performed using a modification of a literature procedure. ${ }^{15}$ To $524 \mathrm{mg}$ (1.39 mmol) of C-6 epimeric alcohols 21 was added $13.9 \mathrm{~mL}$ of a $1 \% \mathrm{w} / \mathrm{v}$ solution of $\mathrm{NaOH}$ in $\mathrm{MeOH}$ at ambient temperature. The mixture was stirred for $1 \mathrm{~h}$ then diluted with brine and the layers were separated. The aqueous layer was extracted with $\mathrm{Et}_{2} \mathrm{O}(3 \mathrm{x})$ and the combined organic extracts were dried $\left(\mathrm{Na}_{2} \mathrm{SO}_{4}\right)$ and concentrated. The resulting crude product mixture was purified by flash chromatography on $\mathrm{SiO}_{2}$ (1:1 hexanes/EtOAc) to afford $373 \mathrm{mg}(94 \%)$ of the product as a clear oil. A sample of the diastereomerically pure benzoate 21 obtained above was subjected to identical reaction conditions to afford diastereomerically pure diol which was fully characterized. IR (thin film) $3353,2957,1656,1247,969,852 \mathrm{~cm}^{-1}$; ${ }^{1} \mathrm{H} \mathrm{NMR}(300 \mathrm{MHz}$, $\left.\mathrm{CDCl}_{3}\right): \delta 5.66(\mathrm{dt}, J=15.6,6.5 \mathrm{~Hz}, 1 \mathrm{H}), 5.53(\mathrm{dd}, J=15.5,6.1 \mathrm{~Hz}, 1 \mathrm{H}), 5.42(\mathrm{dt}, J=15.4,7.8 \mathrm{~Hz}, 1 \mathrm{H}), 5.21$ $(\mathrm{dd}, J=15.2,8.1 \mathrm{~Hz}, 1 \mathrm{H}), 4.17(\mathrm{t}, J=4.9 \mathrm{~Hz}, 1 \mathrm{H}), 3.67(\mathrm{t}, J=6.5 \mathrm{~Hz}, 2 \mathrm{H}), 2.19-2.12(\mathrm{~m}, 1 \mathrm{H}) 2.16(\mathrm{q}, J=6.8$ $\mathrm{Hz}, 2 \mathrm{H}), 1.68$ (q, $J=6.5 \mathrm{~Hz}, 2 \mathrm{H}), 1.44-1.34(\mathrm{~m}, 1 \mathrm{H}), 1.43(\mathrm{br} \mathrm{d}, J=7.3 \mathrm{~Hz}, 2 \mathrm{H}), 0.94(\mathrm{~d}, J=6.8 \mathrm{~Hz}, 3 \mathrm{H}), 0.88$ $(\mathrm{d}, J=6.9 \mathrm{~Hz}, 3 \mathrm{H}), 0.00(\mathrm{~s}, 9 \mathrm{H}) ;{ }^{13} \mathrm{C} \mathrm{NMR}\left(75 \mathrm{MHz}, \mathrm{CDCl}_{3}\right): \delta-1.7,10.7,17.8,22.7,28.6,32.2,39.2,44.1$, 62.4, 74.3, 125.6, 130.6, 133.0, 134.0; MS (EI) m/z $284\left(\mathrm{M}^{+}\right), 269,266,169,141,73$; HRMS (EI) $\mathrm{m} / \mathrm{z}$ calculated for $\mathrm{C}_{16} \mathrm{H}_{32} \mathrm{O}_{2} \mathrm{Si}$ : 284.2172, found 284.2170.

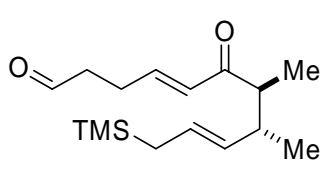

$R^{*-(4 E, 7 S, 8 R, 9 E)-7,8-d i m e t h y l-11-(t r i m e t h y l s i l y l)-6-o x o-u n d e c a-4,9-d i e n a l ~(22): ~ T o ~ a ~}$ $0{ }^{\circ} \mathrm{C}$ solution of $237 \mathrm{mg}(0.833 \mathrm{mmol})$ of the $\mathrm{C}-6$ epimeric alcohols in $8.3 \mathrm{~mL}$ of $\mathrm{CH}_{2} \mathrm{Cl}_{2}$ was added $883 \mathrm{mg}(2.08 \mathrm{mmol})$ of Dess-Martin periodinane. ${ }^{19}$ The reaction was stirred at $0{ }^{\circ} \mathrm{C}$ for $1 \mathrm{~h}$ then slowly warmed to ambient temperature over an additional $3 \mathrm{~h}$. The reaction mixture was diluted with hexanes, filtered through florsil (5:1 hexanes/EtOAc eluent), and concentrated. The resulting crude product mixture was purified by flash chromatography on $\mathrm{SiO}_{2}(5: 1$ hexanes/EtOAc) to afford $0.160 \mathrm{~g}(68 \%)$ of the title compound as a clear oil. Product ratio by GC-MS: $6.1 \%$ (anti-diastereomer or Z-allyl silane $\left.\mathrm{T}_{\mathrm{r}}=14.51\right), 87.8 \%\left(\operatorname{syn} \mathrm{T}_{\mathrm{r}}=14.71\right), 6.1 \%\left(\right.$ anti or Z-allyl silane $\left.\mathrm{T}_{\mathrm{r}}=14.90\right)$. Product ratio by ${ }^{1} \mathrm{H}$ NMR $\left(300 \mathrm{MHz}, \mathrm{Si}\left(\mathrm{C}_{\mathbf{H}_{3}}\right)_{3}\right): 8 \%$ (Z-allyl silane $\delta$ 0.013), $7 \%$ (anti $\left.\delta-0.007\right), 85 \%$ (syn $\left.\delta-0.029\right)$. IR (thin film) 2958, 1727, 1694, 1668, 1628, 1247, 971, $853 \mathrm{~cm}^{-1} ;{ }^{1} \mathrm{H}$ NMR (300 MHz, $\left.\mathrm{CDCl}_{3}\right): \delta 9.91(\mathrm{~s}, 1 \mathrm{H}), 6.80(\mathrm{dt}, J$ $=15.7,6.7 \mathrm{~Hz}, 1 \mathrm{H}), 6.18(\mathrm{dt}, J=15.6,1.4 \mathrm{~Hz}, 1 \mathrm{H}), 5.37(\mathrm{dt}, J=15.2,7.9 \mathrm{~Hz}, 1 \mathrm{H}), 5.15(\mathrm{dd}, J=15.2,7.6 \mathrm{~Hz}$, $1 \mathrm{H}), 2.69-2.42$ (m, 6H), 1.38 (br. d, $J=7.8 \mathrm{~Hz}, 2 \mathrm{H}), 1.02$ (d, $J=6.9 \mathrm{~Hz}, 3 \mathrm{H}), 0.93$ (d, $J=6.8 \mathrm{~Hz}, 3 \mathrm{H}),-0.02$ (s, $9 \mathrm{H}) ;{ }^{13} \mathrm{C}$ NMR $\left(75 \mathrm{MHz}, \mathrm{CDCl}_{3}\right): \delta-2.0,12.8,16.9,22.6,24.7,39.0,42.0,49.9,126.2,130.1,132.1,143.6$,

\footnotetext{
${ }^{15}$ Mashimo, K.; Sato, Y. Tetrahedron, 1970, 26, 803.
} 
200.3, 202.9; MS (EI) $\mathrm{m} / \mathrm{z} 280\left(\mathrm{M}^{+}\right), 265,183,73$; HRMS (EI) $\mathrm{m} / \mathrm{z}$ calculated for $\mathrm{C}_{16} \mathrm{H}_{28} \mathrm{O}_{2} \mathrm{Si}$ : 280.1859 , found 280.1857 .

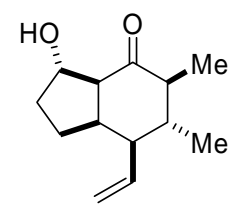

$R^{*}$-(3aS,5S,6R,7R,7aR)-Octahydro-3-hydroxy-5,6-dimethyl-7-vin-ylinden-4-one (23): To a $-78^{\circ} \mathrm{C}$ solution of $65 \mathrm{mg}(23 \mathrm{mmol})$ of ketoaldehyde $22(87.8: 6.1: 6.1$ syn : anti : Z) in $4.6 \mathrm{~mL}$ of $\mathrm{CH}_{2} \mathrm{Cl}_{2}$ was added $0.28 \mathrm{~mL}(0.28 \mathrm{mmol})$ of a $1.0 \mathrm{M} \mathrm{CH}_{2} \mathrm{Cl}_{2}$ solution of $\mathrm{TiCl}_{4}$. After stirring 20 min at $-78{ }^{\circ} \mathrm{C}$, the reaction was quenched by careful addition of $5 \mathrm{ml}$ of sat. aq. $\mathrm{NH}_{4} \mathrm{Cl}$ and the biphasic mixture was warmed to ambient temperature. The aqueous layer was extracted with $\mathrm{CH}_{2} \mathrm{Cl}_{2}(3 \mathrm{x})$ and the combined organic extracts were dried $\left(\mathrm{Na}_{2} \mathrm{SO}_{4}\right)$ and filtered through a fine glass frit with the filter cake being washed with $\mathrm{Et}_{2} \mathrm{O}$. The solvent was evaporated and the resulting crude product mixture was purified by flash chromatography on $\mathrm{SiO}_{2}(2: 1$ hexanes/EtOAc) to give $25 \mathrm{mg}(52 \%)$ of the title compound as a clear, viscous oil. Isolated diastereomeric ratio by GC-MS: $5.78 \%\left(\mathrm{~T}_{\mathrm{r}}=10.15\right), 6.10 \%\left(\mathrm{~T}_{\mathrm{r}}=10.42\right), 5.33 \%\left(\mathrm{~T}_{\mathrm{r}}\right.$ $=11.63), 82.8 \%\left(\mathrm{~T}_{\mathrm{r}}=11.95\right)$. IR (thin film) 3400, 3075, 2969, 1706, 1639, 998, $913 \mathrm{~cm}^{-1} ;{ }^{1} \mathrm{H} \mathrm{NMR}(300 \mathrm{MHz}$, $\left.\mathrm{CDCl}_{3}\right): \delta 5.59(\mathrm{dt}, J=17.1,9.8 \mathrm{~Hz}, 1 \mathrm{H}), 5.12(\mathrm{dd}, J=17.0,1.9 \mathrm{~Hz}, 1 \mathrm{H}), 5.09(\mathrm{dd}, J=9.9,1.9 \mathrm{~Hz}, 1 \mathrm{H}), 4.83(\mathrm{dd}$, $J=6.4,2.3 \mathrm{~Hz}, 1 \mathrm{H}), 2.85-2.73(\mathrm{~m}, 2 \mathrm{H}), 2.48(\mathrm{td}, J=10.3,4.7 \mathrm{~Hz}, 1 \mathrm{H}), 2.20-2.09(\mathrm{~m}, 2 \mathrm{H}), 1.81-1.71(\mathrm{~m}, 1 \mathrm{H})$, 1.58-1.40 (m, 3H), 1.26-1.09 (m, 1H), $1.03(\mathrm{~d}, \mathrm{~J}=6.5 \mathrm{~Hz}, 3 \mathrm{H}), 0.99(\mathrm{~d}, \mathrm{~J}=6.4 \mathrm{~Hz}, 3 \mathrm{H}) ;{ }^{13} \mathrm{C} \mathrm{NMR}(75 \mathrm{MHz}$, $\left.\mathrm{CDCl}_{3}\right): \delta 11.7,18.7,23.7,33.1,39.4,46.6,49.2,50.1,61.2,72.3,116.2,140.5,212.2 ; \mathrm{MS}(\mathrm{EI}) \mathrm{m} / \mathrm{z} 208\left(\mathrm{M}^{+}\right)$, 190, 140, 122, 68; HRMS (EI) $\mathrm{m} / \mathrm{z}$ calculated for $\mathrm{C}_{13} \mathrm{H}_{20} \mathrm{O}_{2}: 208.1463$, found 208.1468.

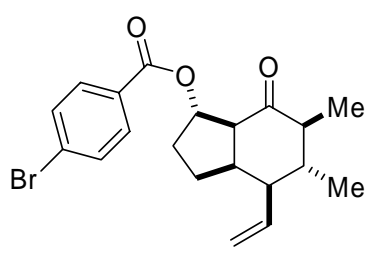

$R^{*}$-(3aS,5S,6R,7R,7aR)-Octahydro-5,6-dimethyl-4-oxo-7-vinyl-1H-inden-3-yl 4bromobenzoate (24): To a $0{ }^{\circ} \mathrm{C}$ solution of $25 \mathrm{mg}(0.12 \mathrm{mmol})$ of hydrindanone 23 in $1.2 \mathrm{~mL}$ of $\mathrm{CH}_{2} \mathrm{Cl}_{2}$ was added $1.5 \mathrm{mg}(0.012 \mathrm{mmol})$ of 4-(dimethylamino)pyridine, $31.4 \mathrm{mg}(0.143 \mathrm{mmol})$ of 4-bromobenzoyl chloride and $25 \mu \mathrm{L}(19 \mathrm{mg}, 0.15 \mathrm{mmol})$ of $N, N$-diisopropylethylamine. The reaction was warmed ambient temperature and stirred $24 \mathrm{~h}$. The reaction mixture was poured into aq. $1 \mathrm{M} \mathrm{HCl}$ and extracted with EtOAc $(4 \mathrm{x})$. The combined organic extracts were dried $\left(\mathrm{Na}_{2} \mathrm{SO}_{4}\right)$, concentrated and the crude product mixture was purified by flash chromatography on $\mathrm{SiO}_{2}(2 \mathrm{x})$ (2:1 hexanes/EtOAc then 10:1 hexanes/EtOAc) to afford 26 mg $(55 \%)$ of the title compound as a colorless crystalline solid: m.p. $98-100{ }^{\circ} \mathrm{C}$. Recrystallizing this solid from pentane/ $\mathrm{Et}_{2} \mathrm{O}$ (slow evaporation) gave crystals suitable for X-ray diffraction analysis. IR (thin film) 3090, 2971, 1718, 1590, $1270 \mathrm{~cm}^{-1} ;{ }^{1} \mathrm{H}$ NMR $\left(300 \mathrm{MHz}, \mathrm{CDCl}_{3}\right): \delta 7.86(\mathrm{~d}, J=8.5 \mathrm{~Hz}, 2 \mathrm{H}), 7.57(\mathrm{~d}, J=8.5 \mathrm{~Hz}, 2 \mathrm{H}), 5.90$ $(\mathrm{dd}, J=7.1,1.9 \mathrm{~Hz}, 1 \mathrm{H}), 5.61(\mathrm{dt}, J=17.1,9.8 \mathrm{~Hz}, 1 \mathrm{H}), 5.15(\mathrm{dd}, J=17.1,1.8 \mathrm{~Hz}, 1 \mathrm{H}), 5.12(\mathrm{dd}, J=10.0,1.9$ $\mathrm{Hz}, 1 \mathrm{H}), 3.05(\mathrm{~d}, J=7.3 \mathrm{~Hz}, 1 \mathrm{H}), 2.82-2.72(\mathrm{~m}, 1 \mathrm{H}), 2.52(\mathrm{td}, J=10.6,5.1 \mathrm{~Hz}, 1 \mathrm{H}), 2.41-2.27(\mathrm{~m}, 1 \mathrm{H}), 2.23-$ $2.13(\mathrm{~m}, 1 \mathrm{H}), 1.86-1.66(\mathrm{~m}, 2 \mathrm{H}), 1.62-1.48(\mathrm{~m}, 1 \mathrm{H}), 1.33-1.18(\mathrm{~m}, 1 \mathrm{H}), 1.05(\mathrm{~d}, J=6.5 \mathrm{~Hz}, 3 \mathrm{H}), 1.00(\mathrm{~d}, J=6.4$ $\mathrm{Hz}, 3 \mathrm{H}) ;{ }^{13} \mathrm{C}$ NMR $\left(75 \mathrm{MHz}, \mathrm{CD}_{3} \mathrm{CN}\right): \delta 12.1,19.0,24.3,31.0,40.4,48.5,49.7,50.6,59.5,77.4,116.7,128.4$, 130.9, 132.1, 132.8, 142.0, 166.1, 211.6; MS (ESI) $\mathrm{m} / \mathrm{z} 413\left(\mathrm{M}^{+}+\mathrm{Na}\right)$; HRMS (ESI) $\mathrm{m} / \mathrm{z}$ calculated for $\mathrm{NaC}_{20} \mathrm{H}_{23} \mathrm{O}_{3} \mathrm{Br}\left(\mathrm{M}^{+}+\mathrm{Na}\right):$ 413.0728, found 413.0749. 


\section{X-ray Structure Determinations for Compounds 19 and 24}

\section{Compound 19}

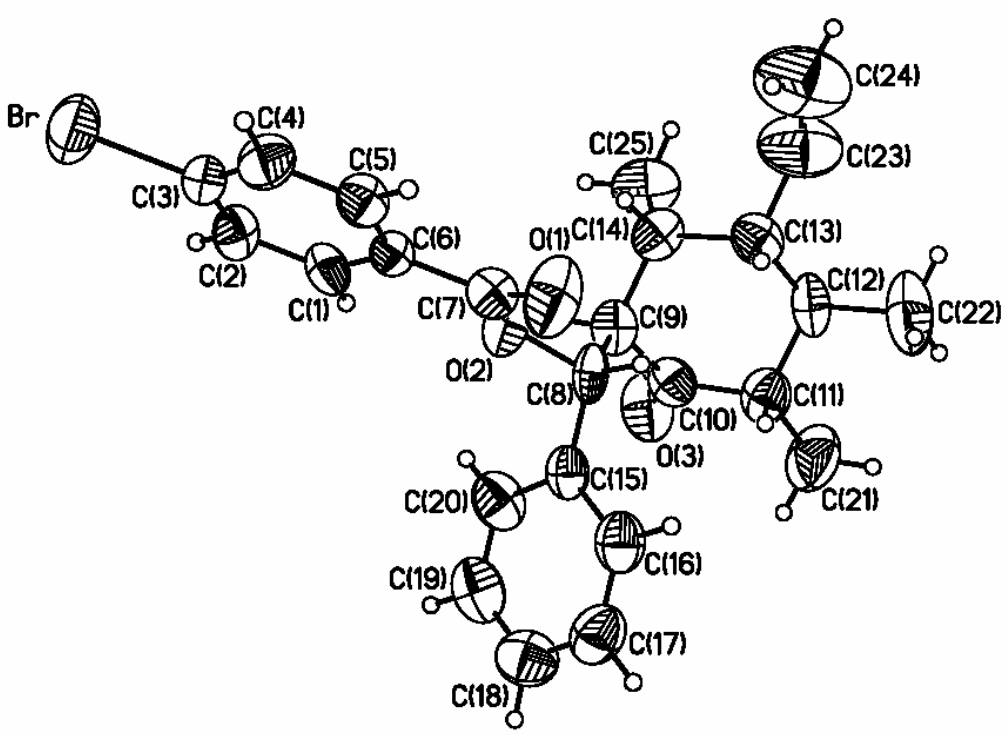

Figure S1. Crystal Structure of $R^{*}-(R)-((1 R, 2 R, 3 R, 4 R, 5 S)-2,4,5$-Trimethyl-6-oxo3 -vinylcyclohexyl)(phenyl)methyl 4-bromobenzoate (19)

Table 1. Crystal data and structure refinement for $\mathbf{1 9}$

Identification code

Empirical formula

Formula weight

Temperature

Wavelength

Crystal system

Space group

Unit cell dimensions

Volume

Z

Density (calculated)

Absorption coefficient

$\mathrm{F}(000)$

Crystal size

Theta range for data collection

Index ranges

Reflections collected

Independent reflections

Completeness to theta $=25.00^{\circ}$

Absorption correction

Max. and min. transmission

Refinement method

Data / restraints / parameters

Goodness-of-fit on $\mathrm{F}^{2}$

Final $\mathrm{R}$ indices [I $>2 \operatorname{sigma}(\mathrm{I})]$

$\mathrm{R}$ indices (all data)

Largest diff. peak and hole

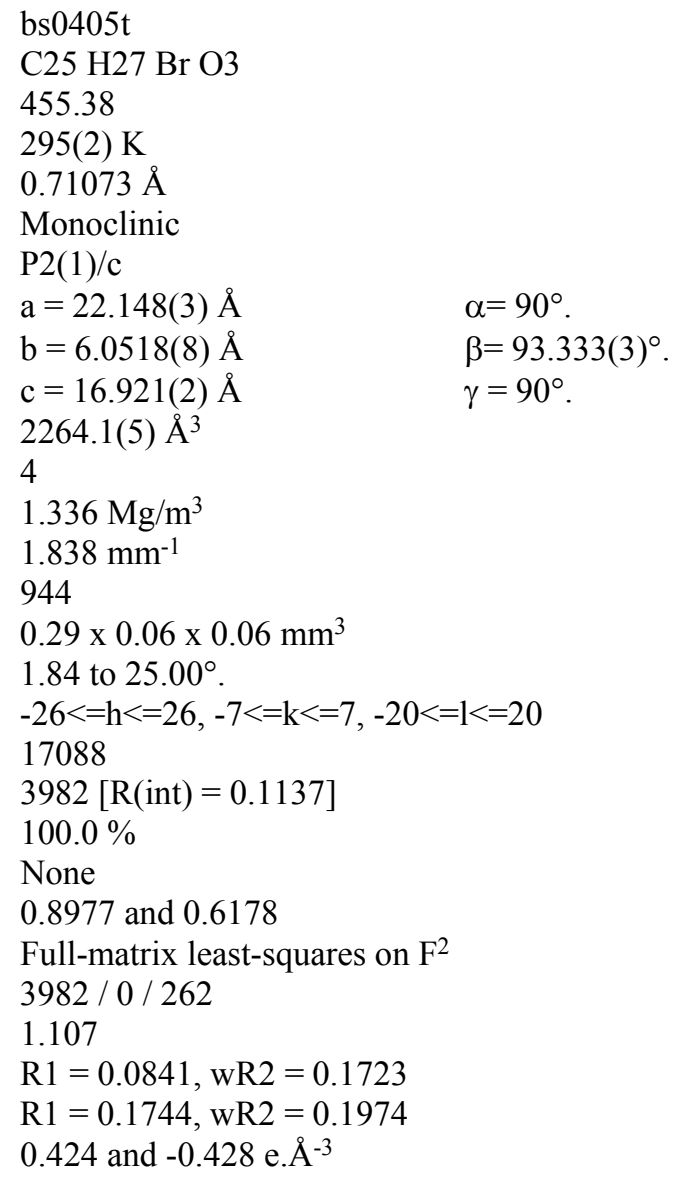




\section{Compound 24}

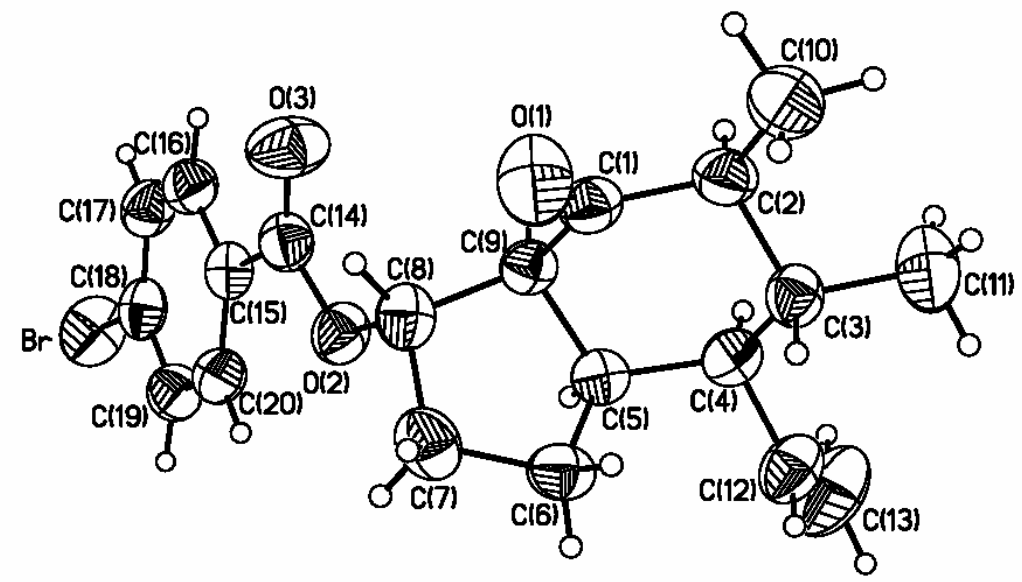

Figure S2. Crystal Structure of $R^{*}-(3 \mathrm{a} S, 5 S, 6 R, 7 R, 7 \mathrm{a} R)$-Octahydro-5,6-dimethyl-4-oxo-7vinyl-1H-inden-3-yl 4-bromobenzoate (24)

Table 1. Crystal data and structure refinement for $\mathbf{2 4}$

Identification code

Empirical formula

Formula weight

Temperature

Wavelength

Crystal system

Space group

Unit cell dimensions

Volume

Z

Density (calculated)

Absorption coefficient

$\mathrm{F}(000)$

Crystal size

Theta range for data collection

Index ranges

Reflections collected

Independent reflections

Completeness to theta $=32.50^{\circ}$

Absorption correction

Max. and min. transmission

Refinement method

Data / restraints / parameters bs03171s

C20 H23 Br O3

391.29

295(2) K

$0.71073 \AA$

Monoclinic

$\mathrm{P} 2(1) / \mathrm{c}$

$\mathrm{a}=8.0654(4) \AA$

$\alpha=90^{\circ}$.

$\mathrm{b}=22.8205(13) \AA$

$\mathrm{c}=10.8106(6) \AA$

$\beta=111.4580(10)^{\circ}$.

1851.84(17) $\AA^{3}$

4

$1.403 \mathrm{Mg} / \mathrm{m}^{3}$

$2.234 \mathrm{~mm}^{-1}$

808

$0.33 \times 0.14 \times 0.03 \mathrm{~mm}^{3}$

1.78 to $32.50^{\circ}$.

$-11<=\mathrm{h}<=12,-34<=\mathrm{k}<=34,-16<=\mathrm{l}<=16$

24011

6565 [R(int) $=0.0489]$

$98.0 \%$

Sadabs

0.9401 and 0.5260

Full-matrix least-squares on $\mathrm{F}^{2}$

6565 / 0 / 309 


\section{Goodness-of-fit on $\mathrm{F}^{2}$}

Final $\mathrm{R}$ indices [I $>2 \operatorname{sigma}(\mathrm{I})]$

$\mathrm{R}$ indices (all data)

Largest diff. peak and hole

\subsection{7}

$\mathrm{R} 1=0.0523, \mathrm{wR} 2=0.1127$

$\mathrm{R} 1=0.1259, \mathrm{wR} 2=0.1394$

0.447 and -0.179 e. $\AA^{-3}$ 


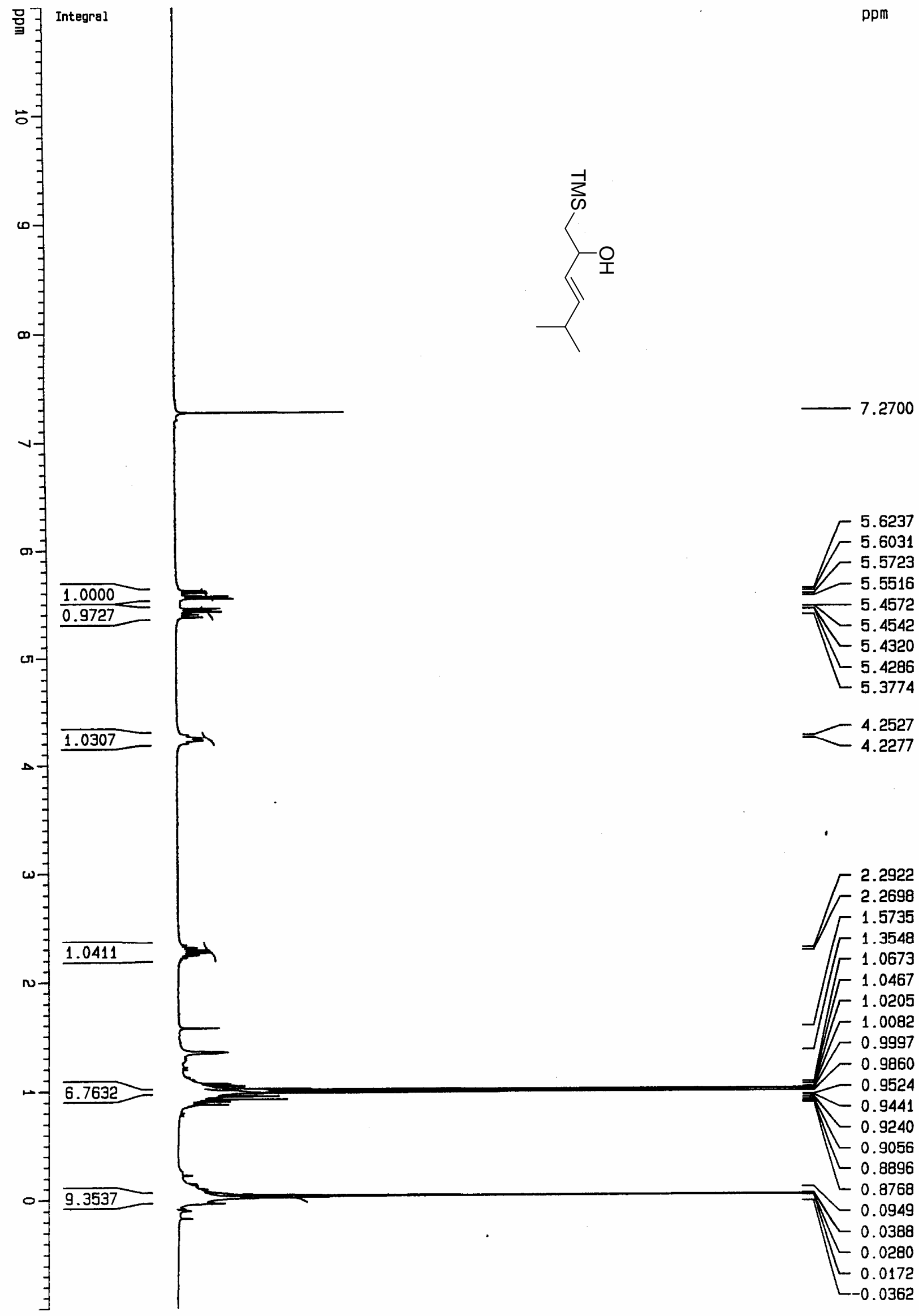




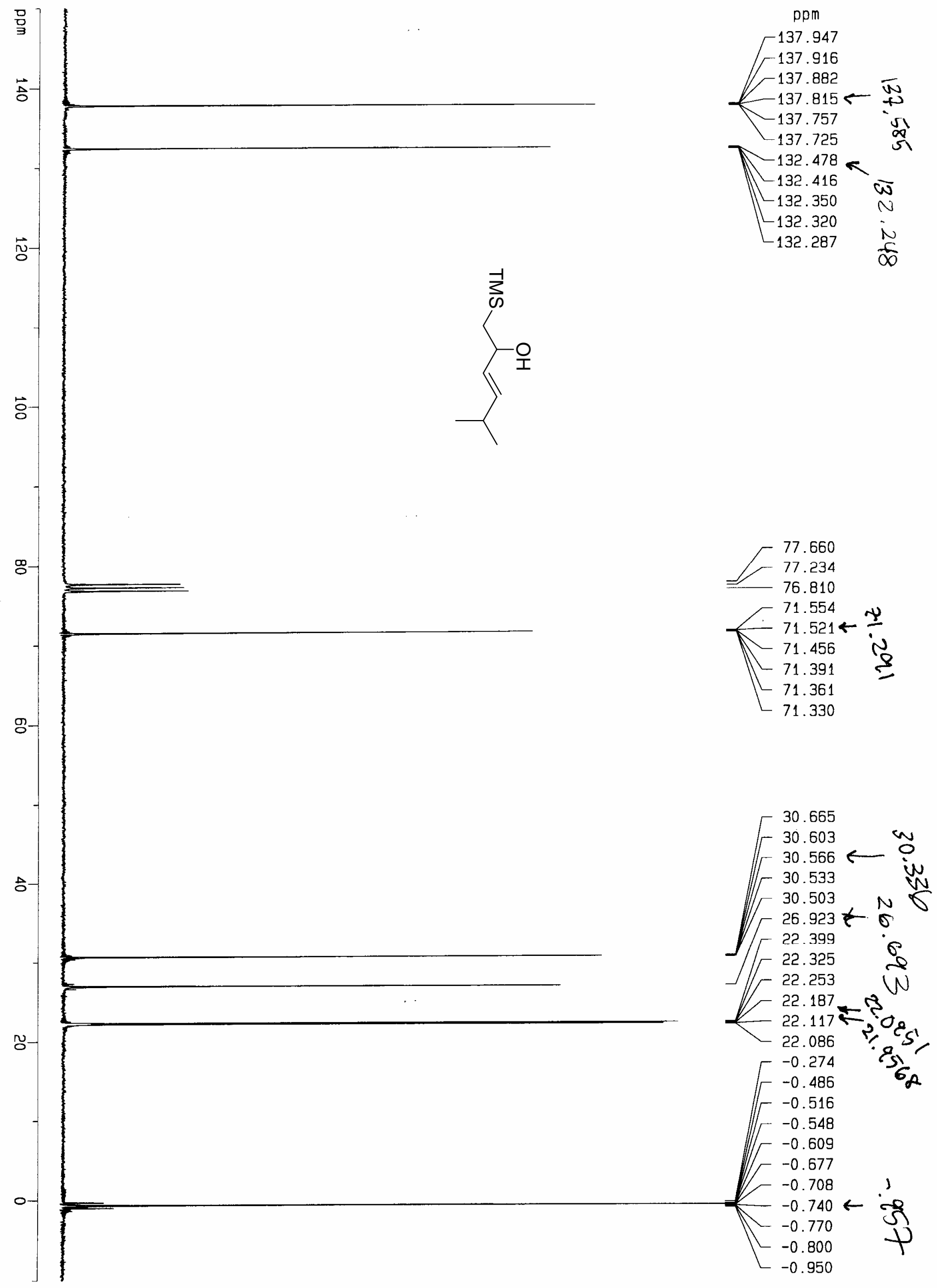




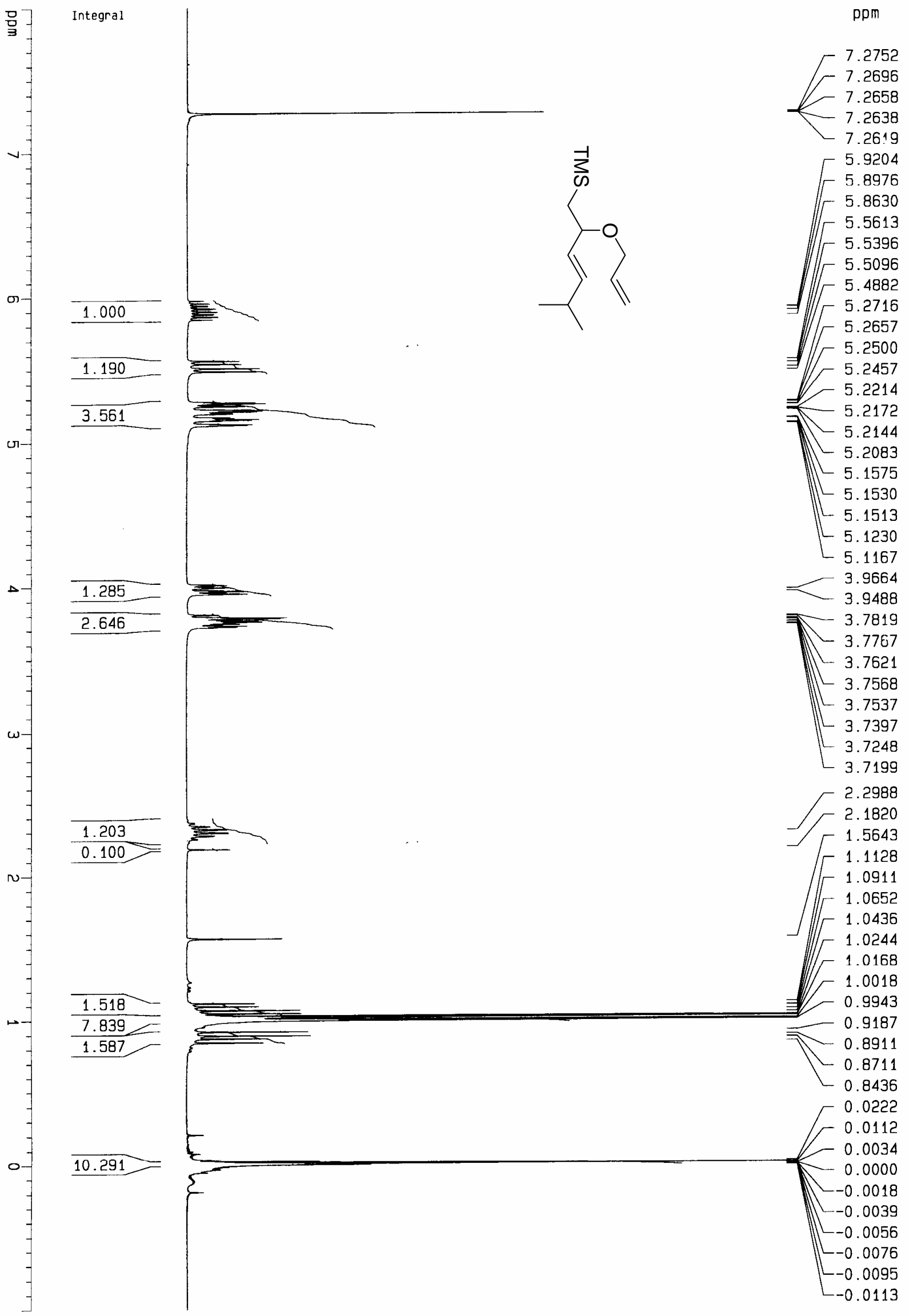




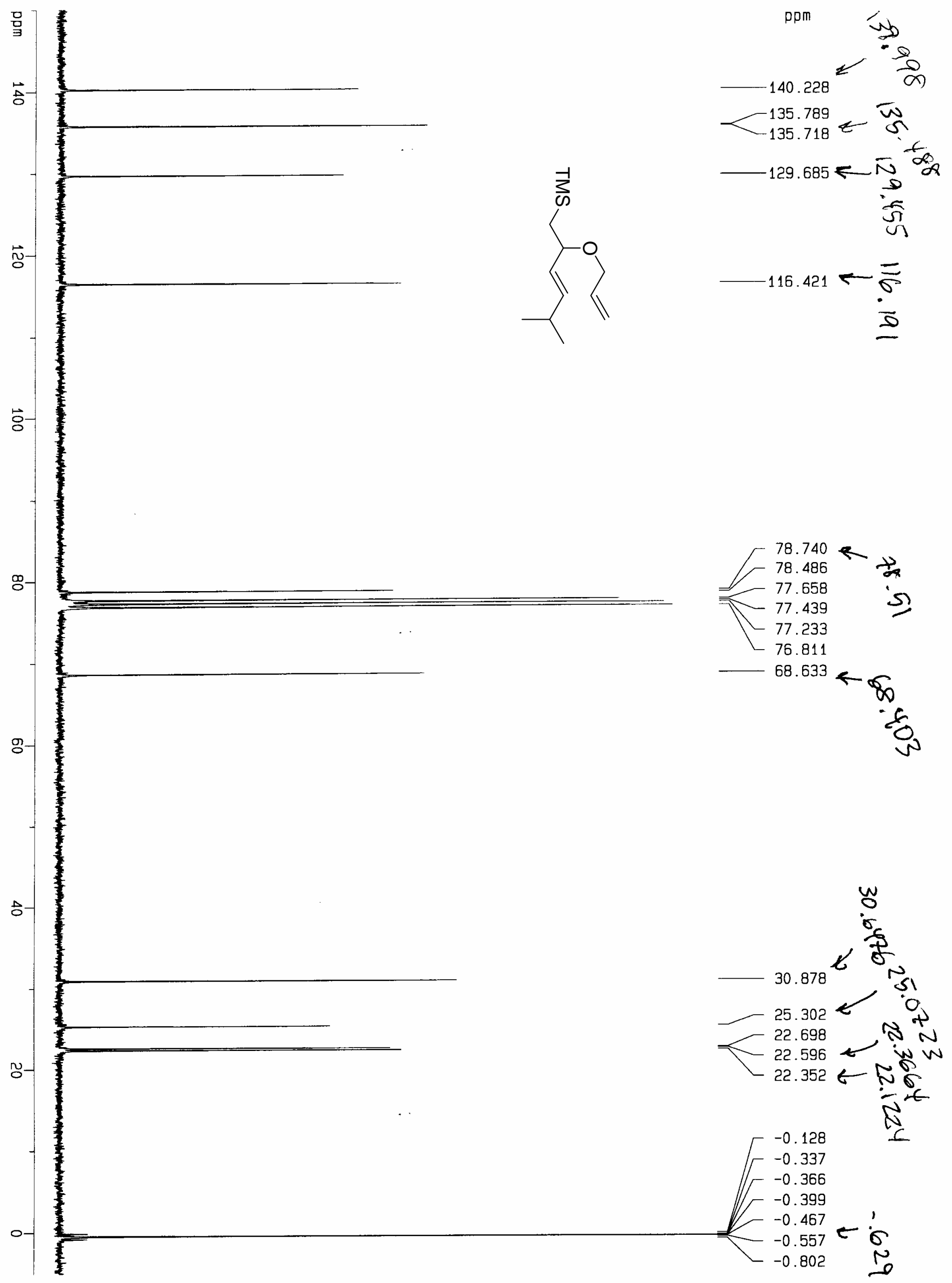




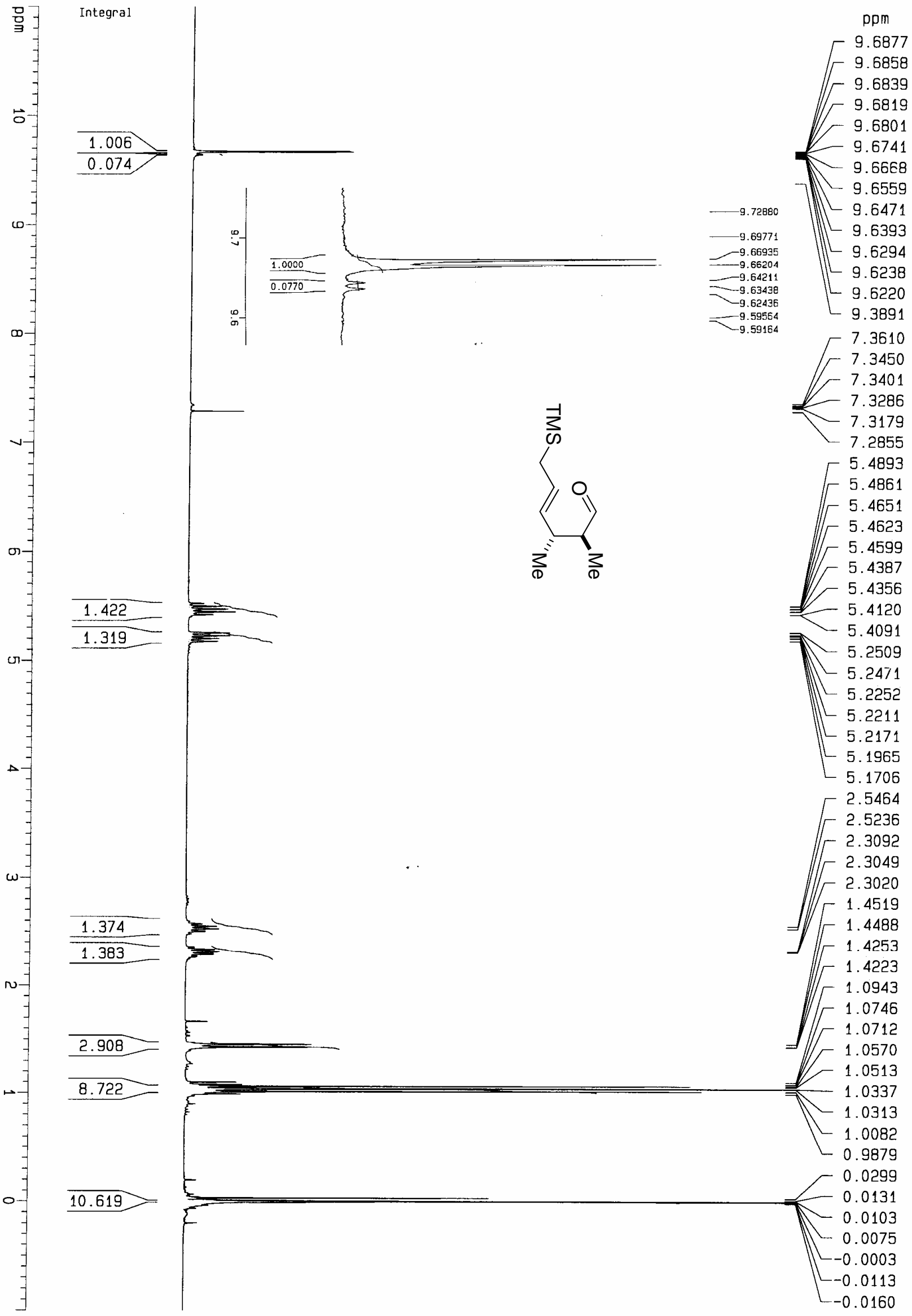




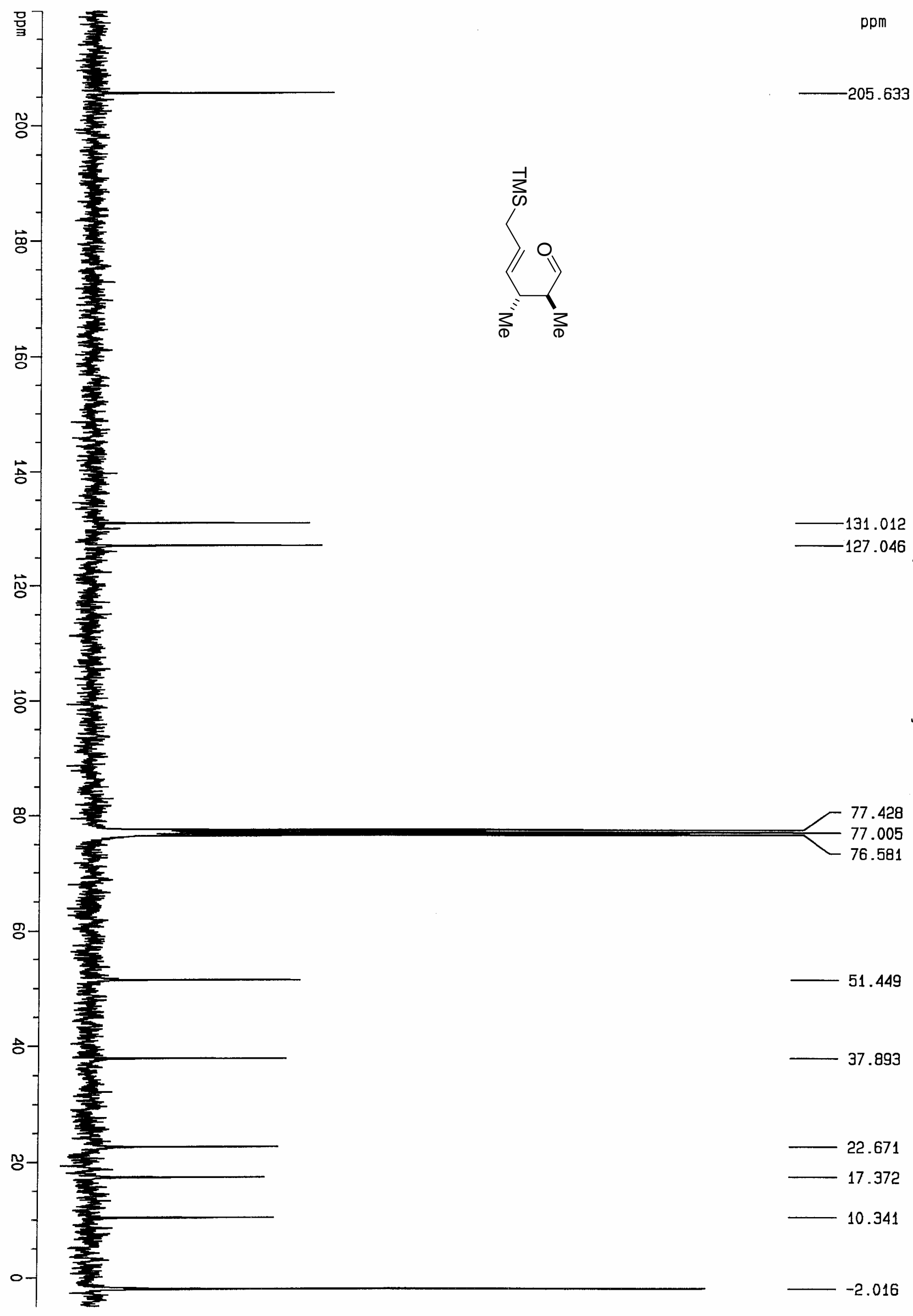




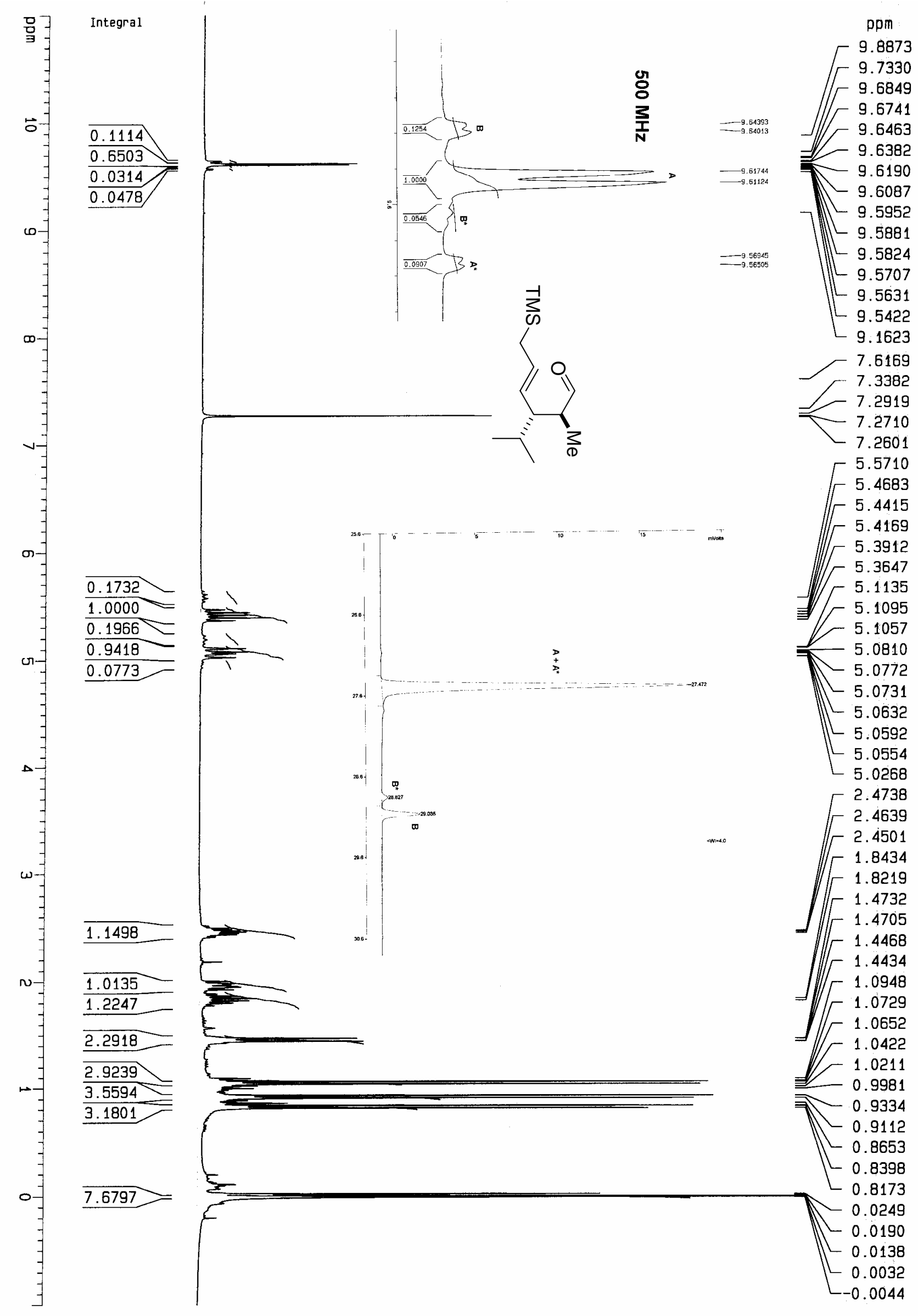




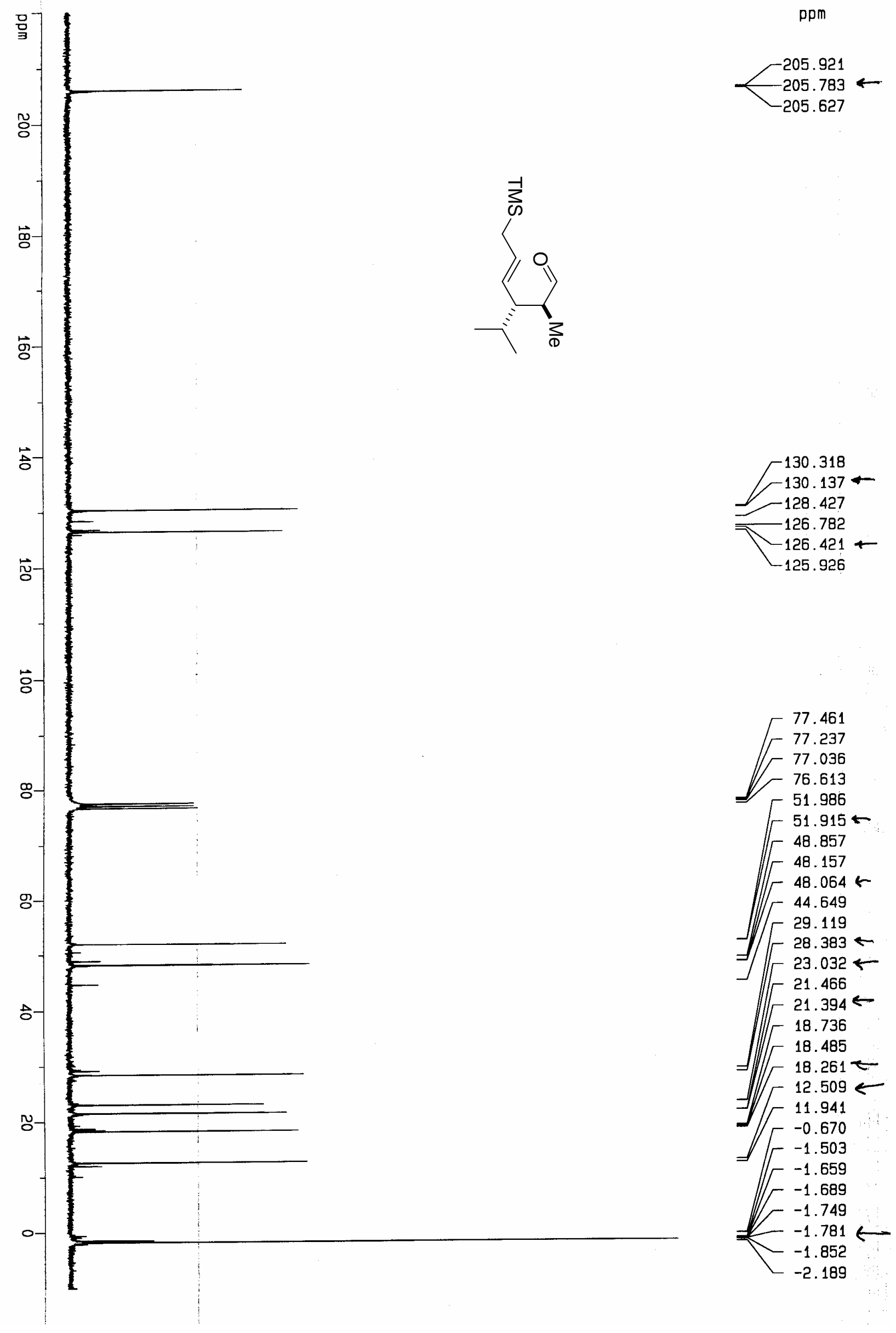




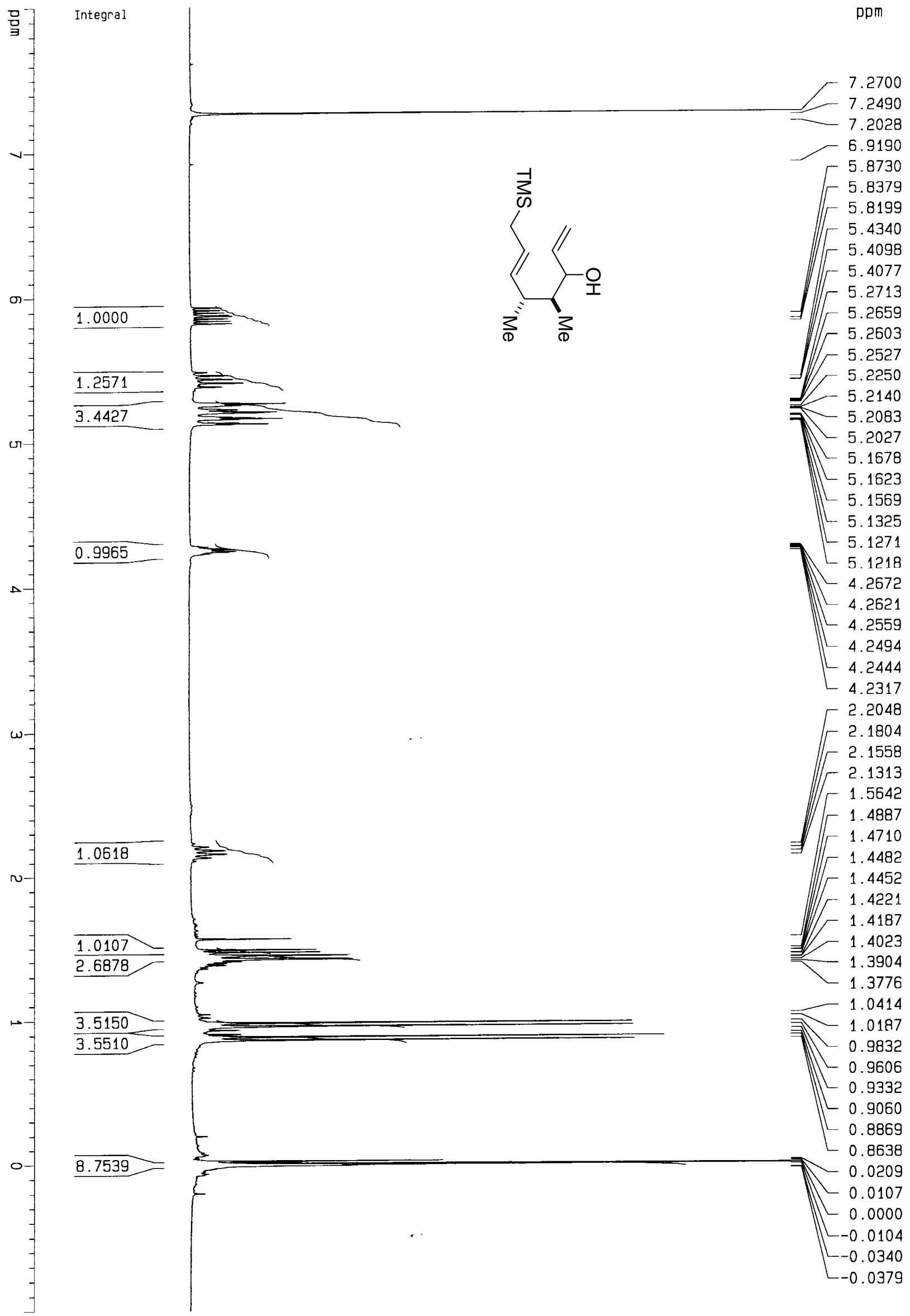




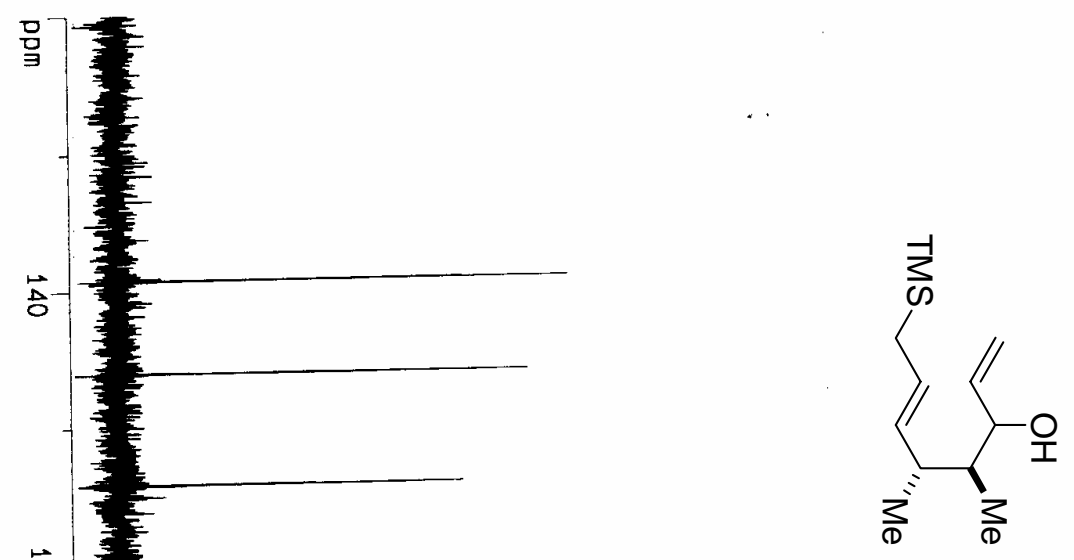

ppm
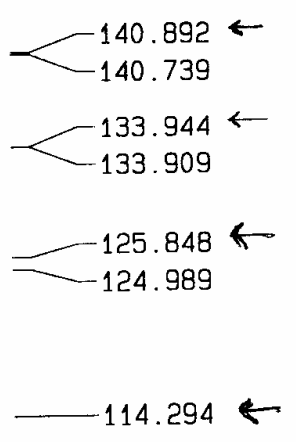

$-106.386$
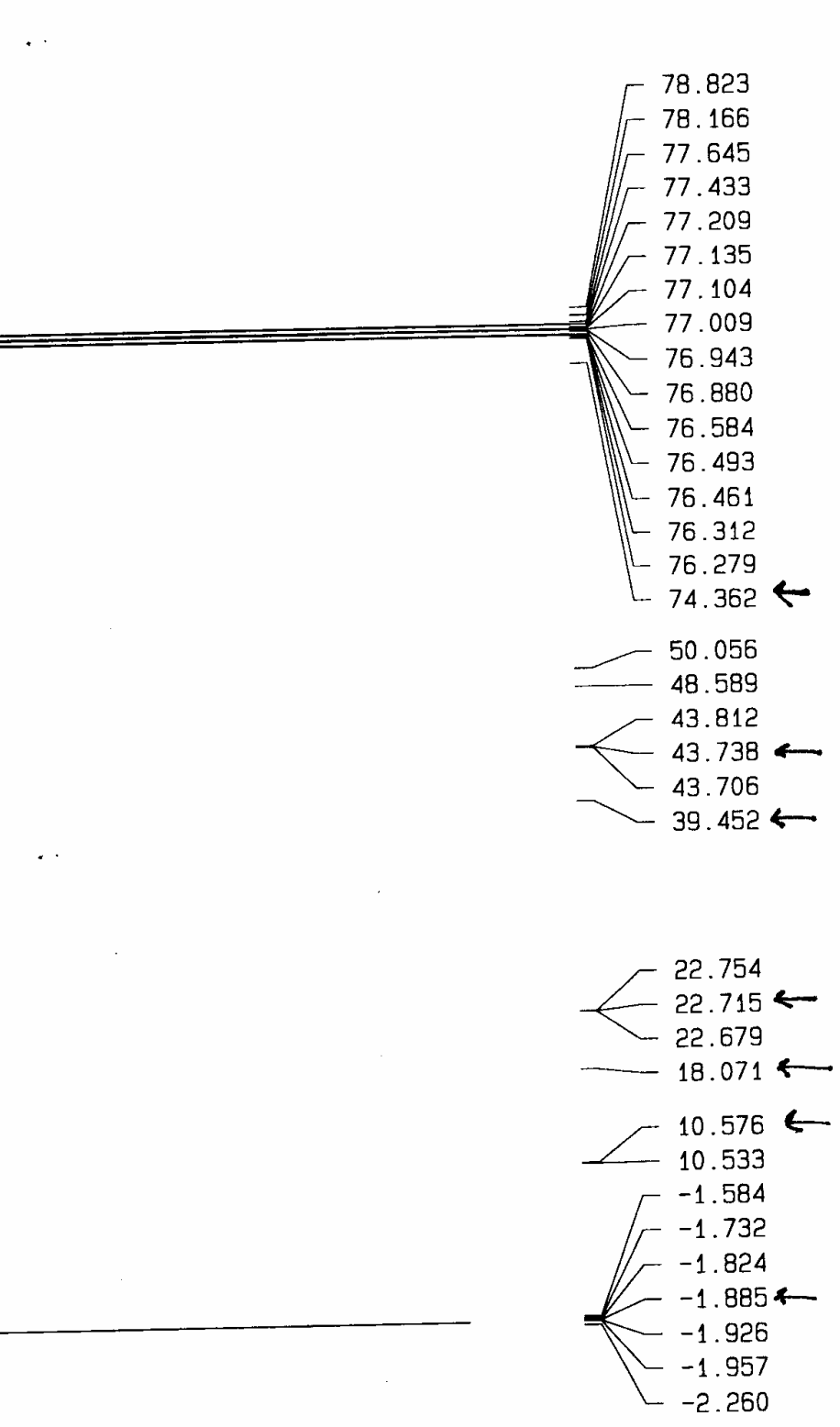

-S 26 - 


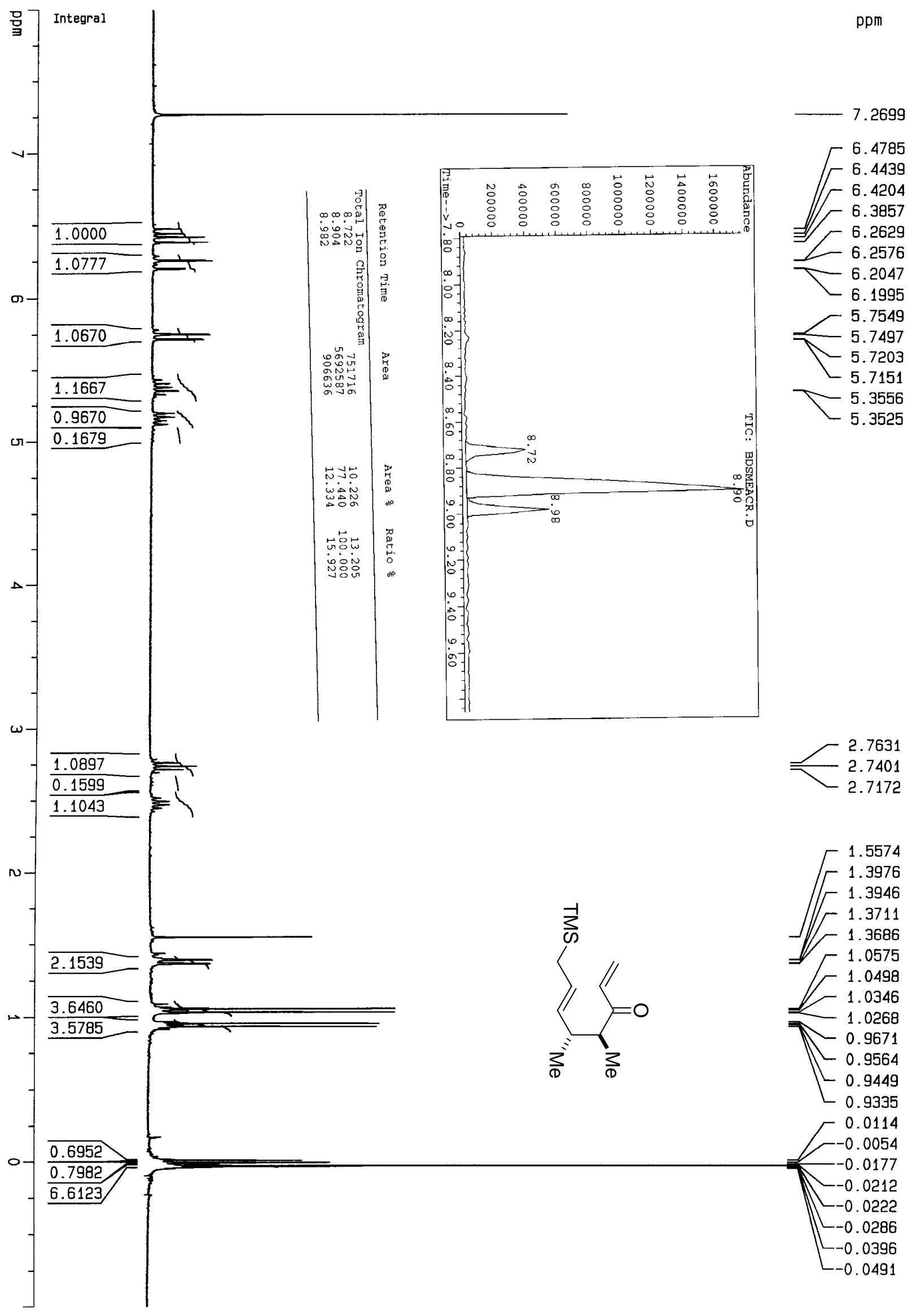



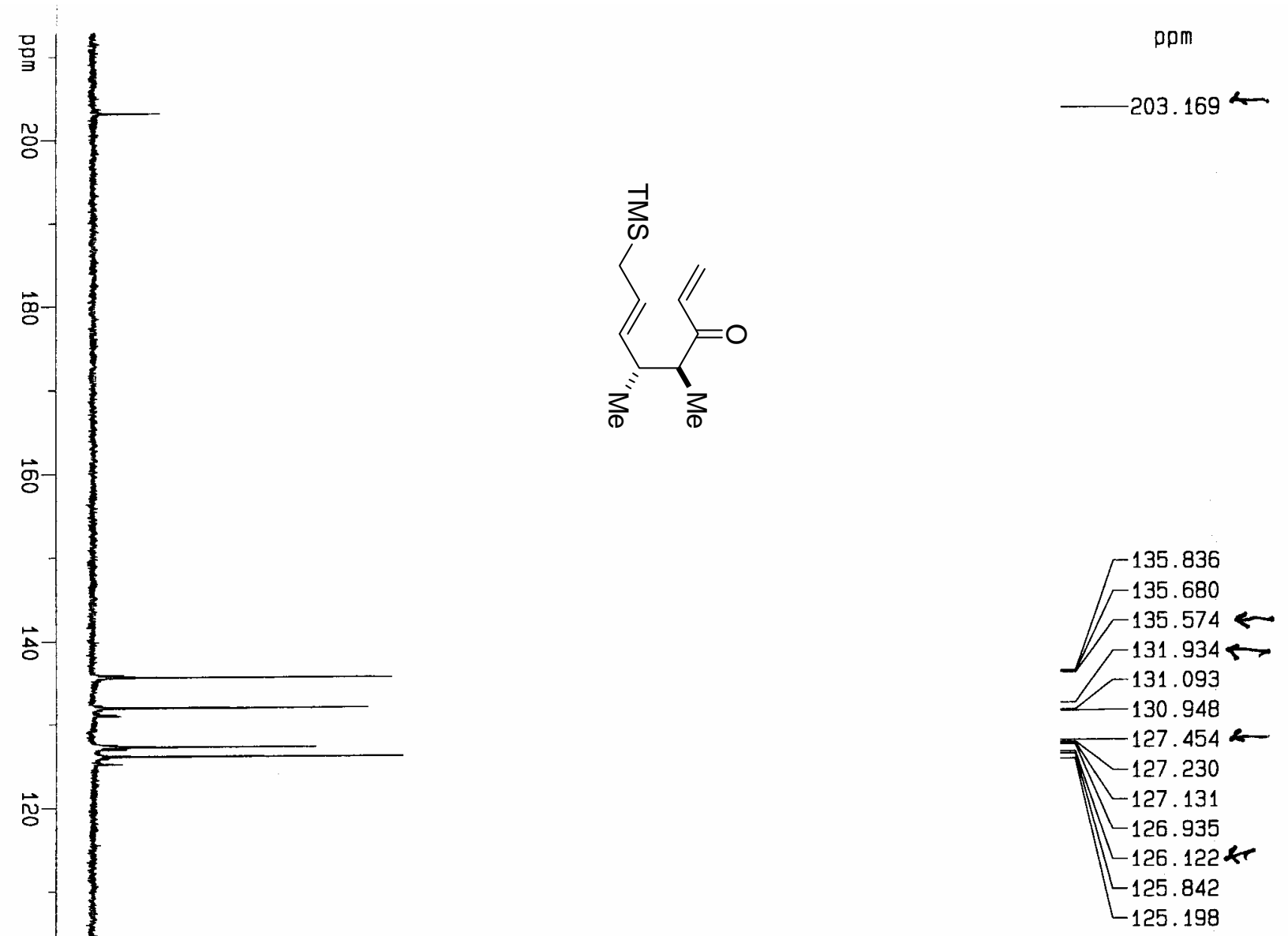

s

용

항

客

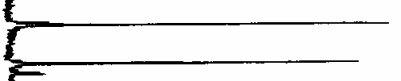

宫

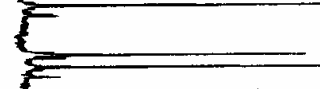

:

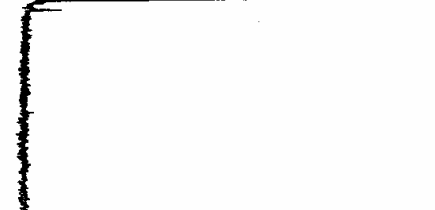

8

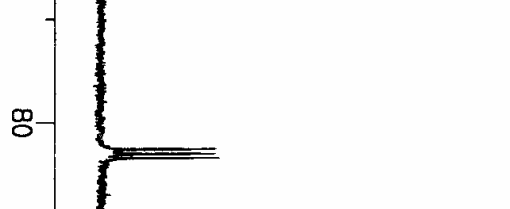

용

앙-

$+$

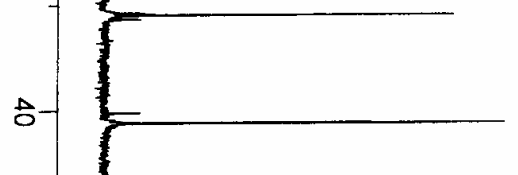

ก
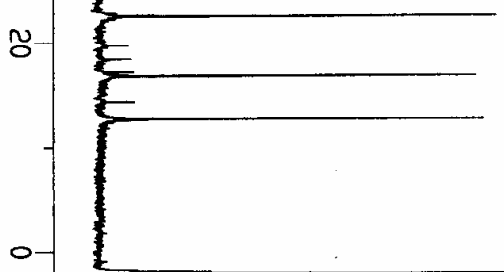

r

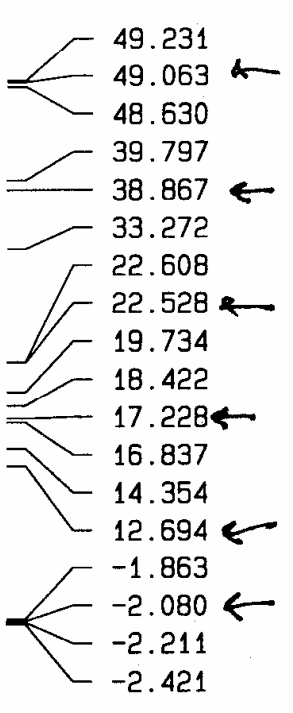




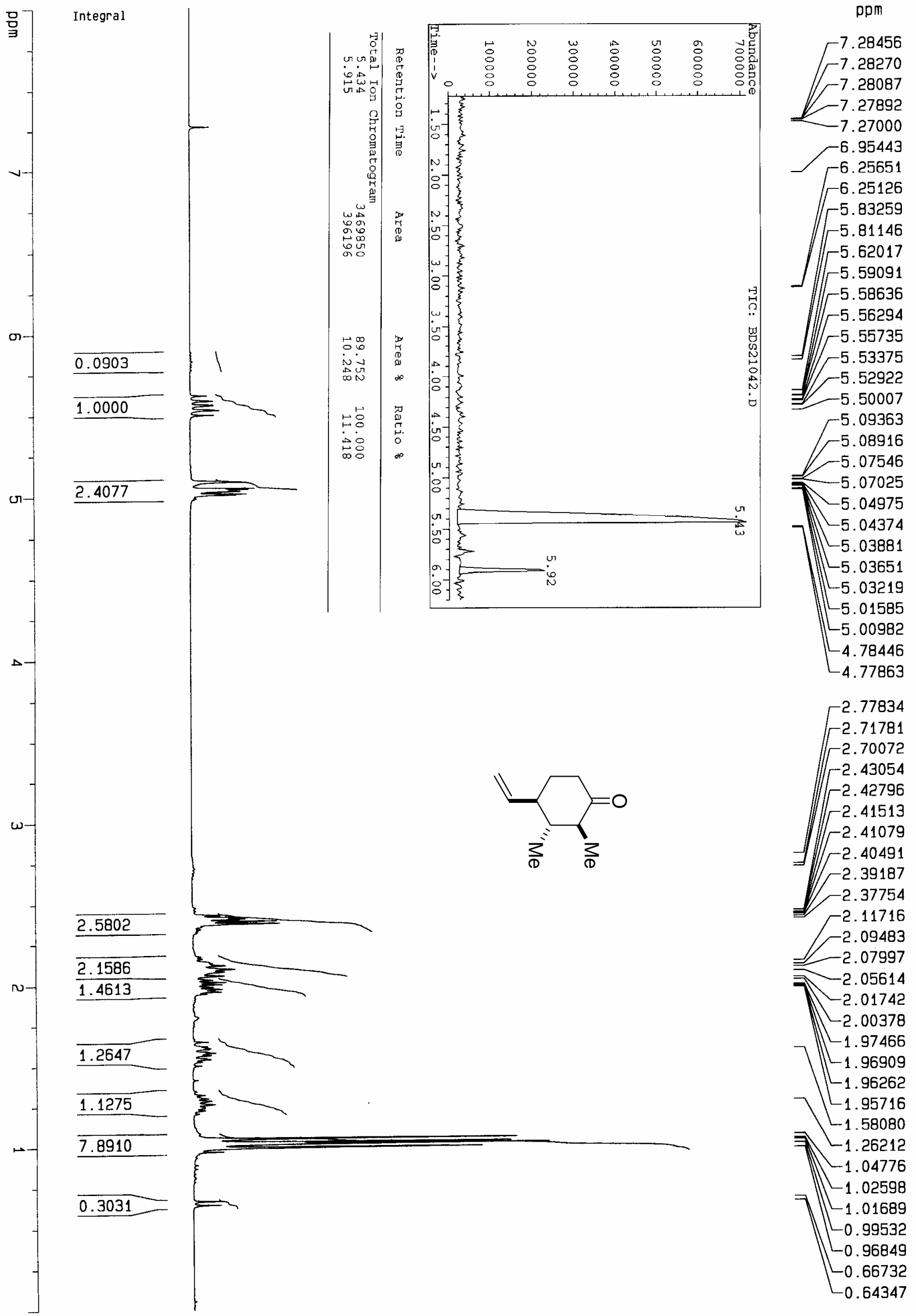



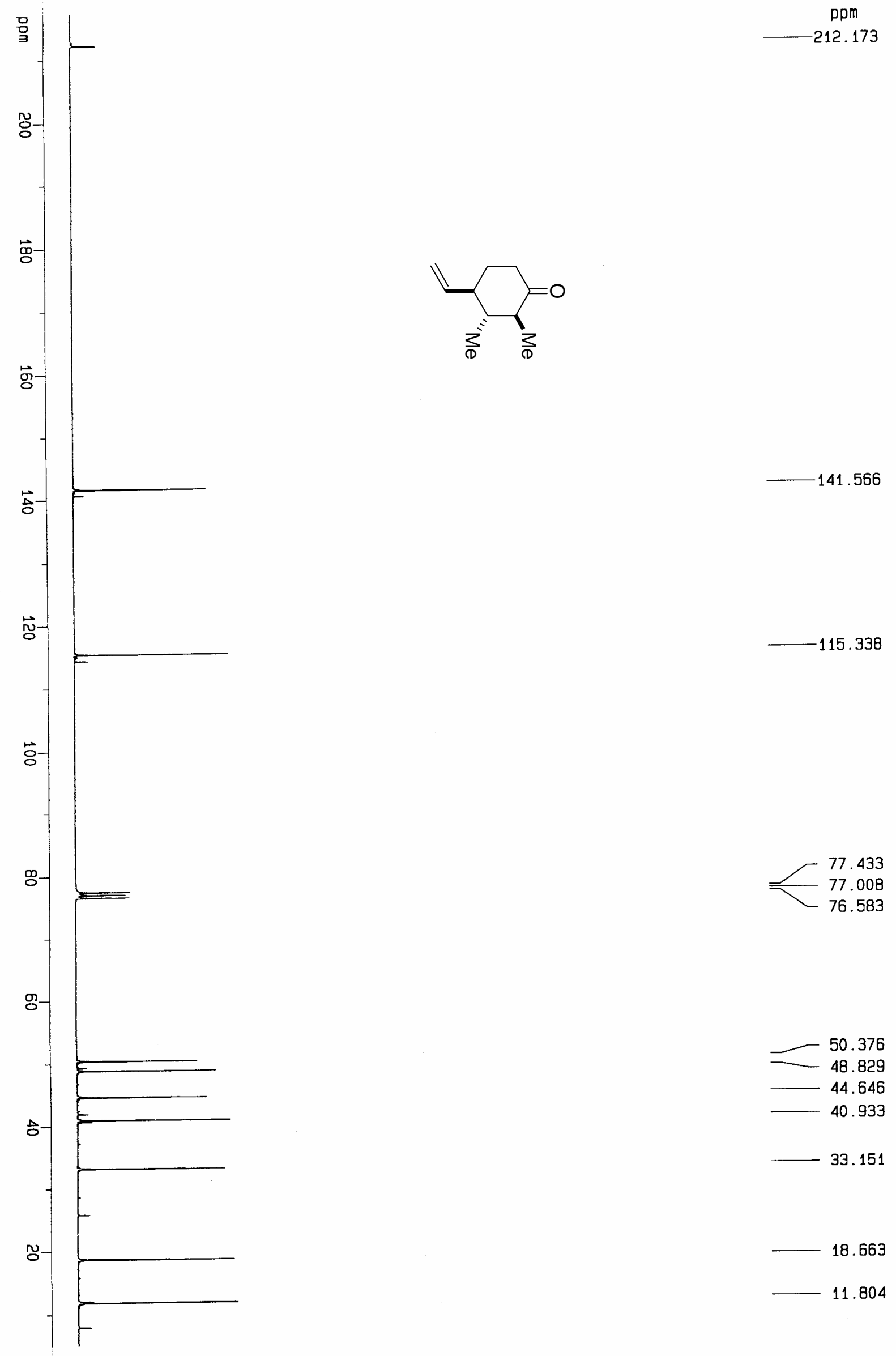


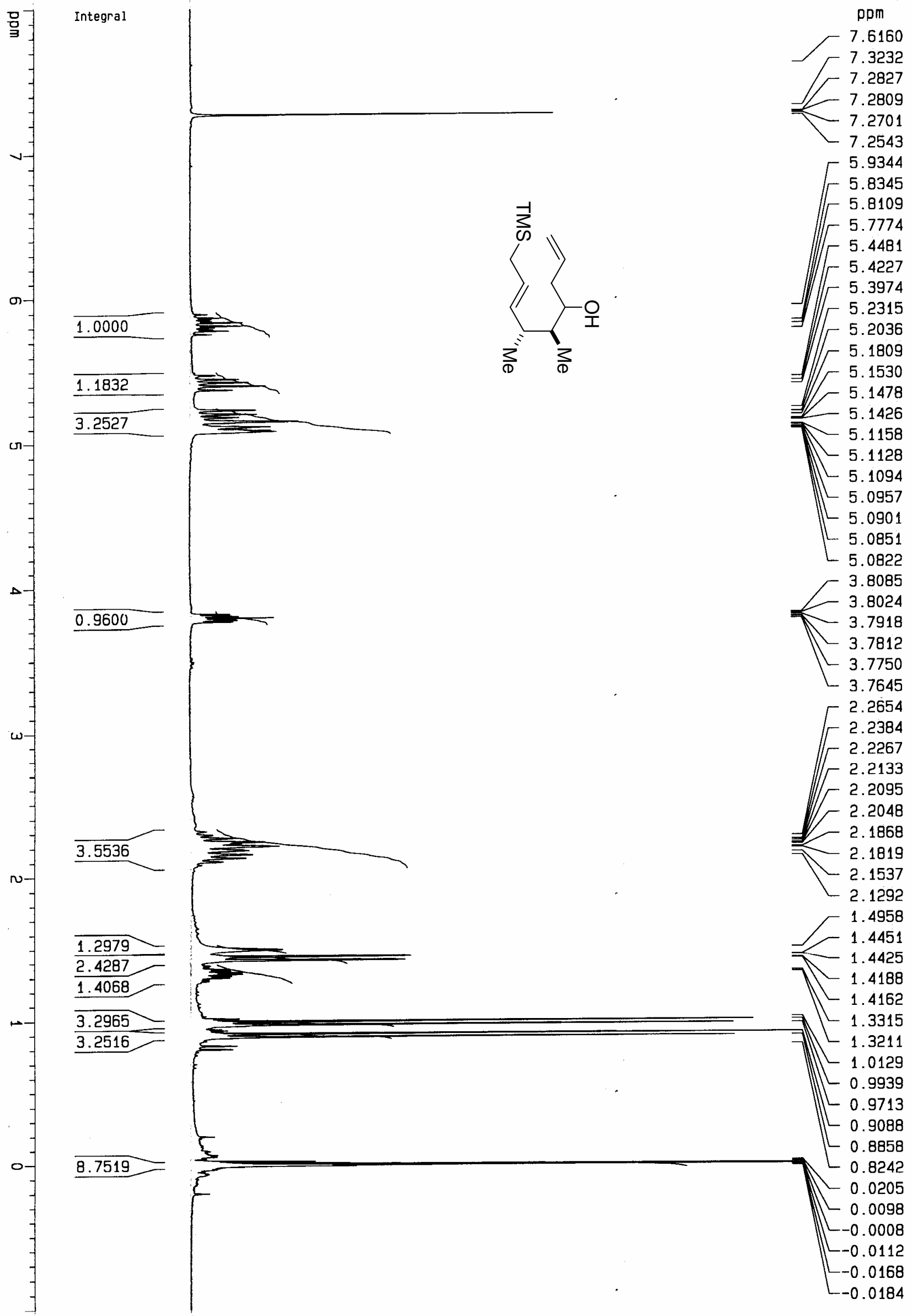



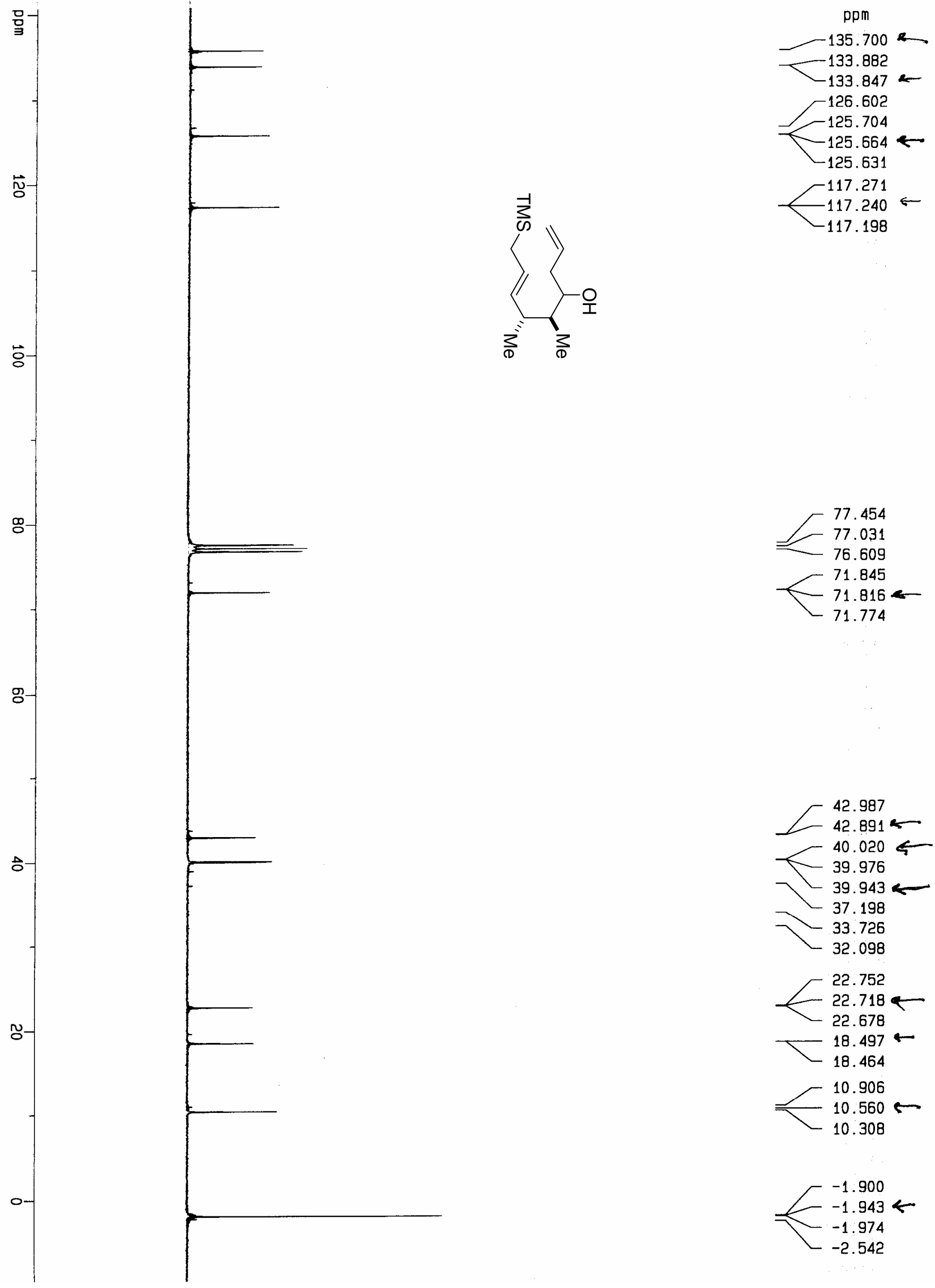


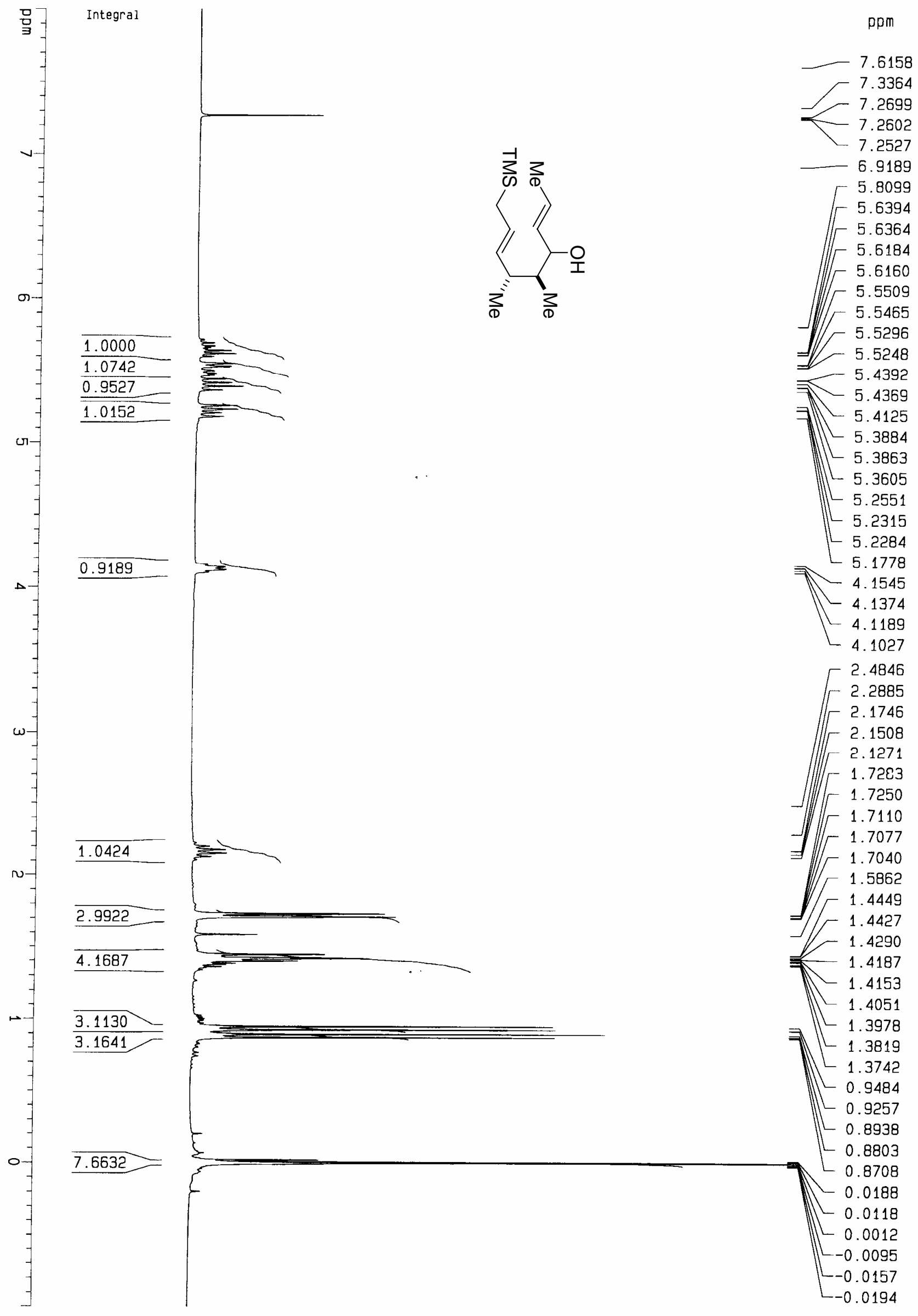




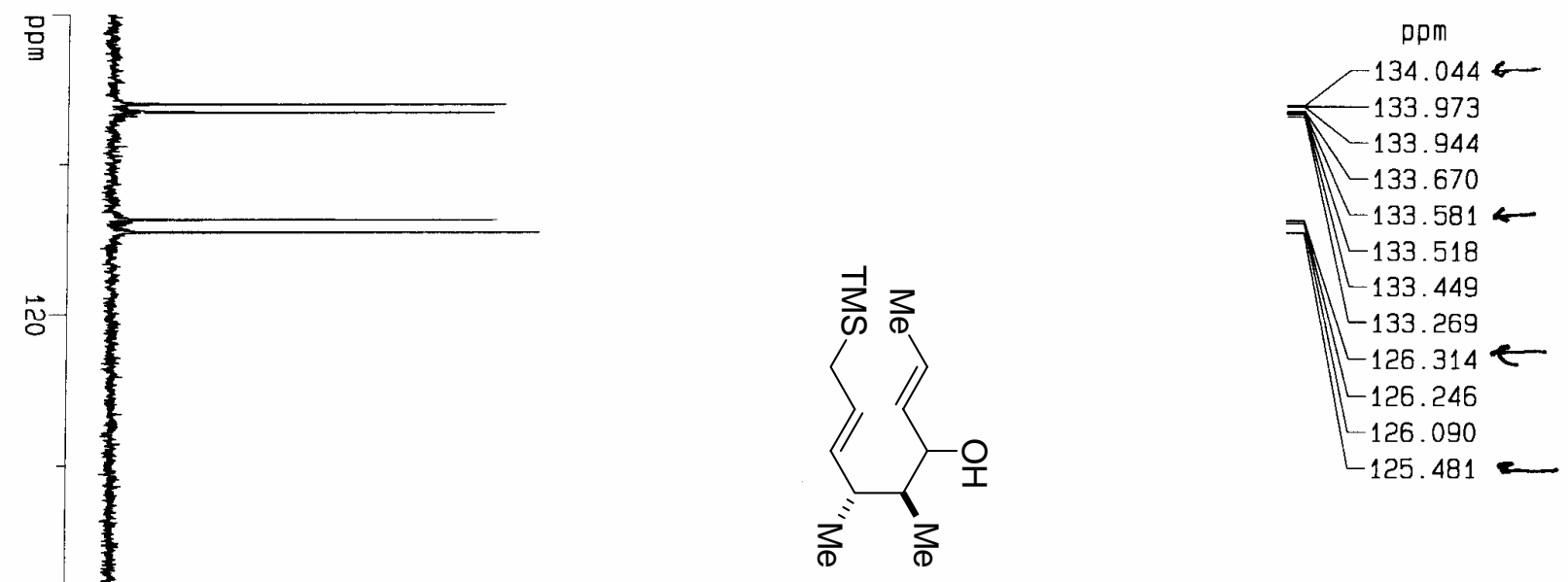

용

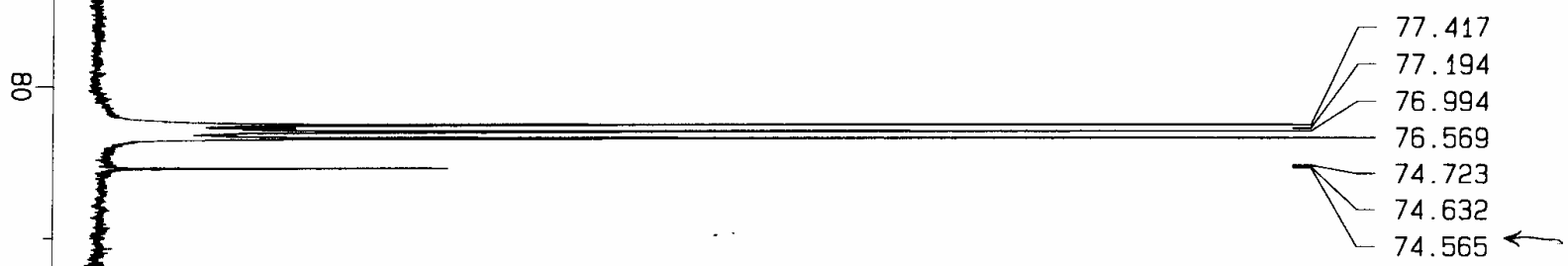

용

o

ก

)
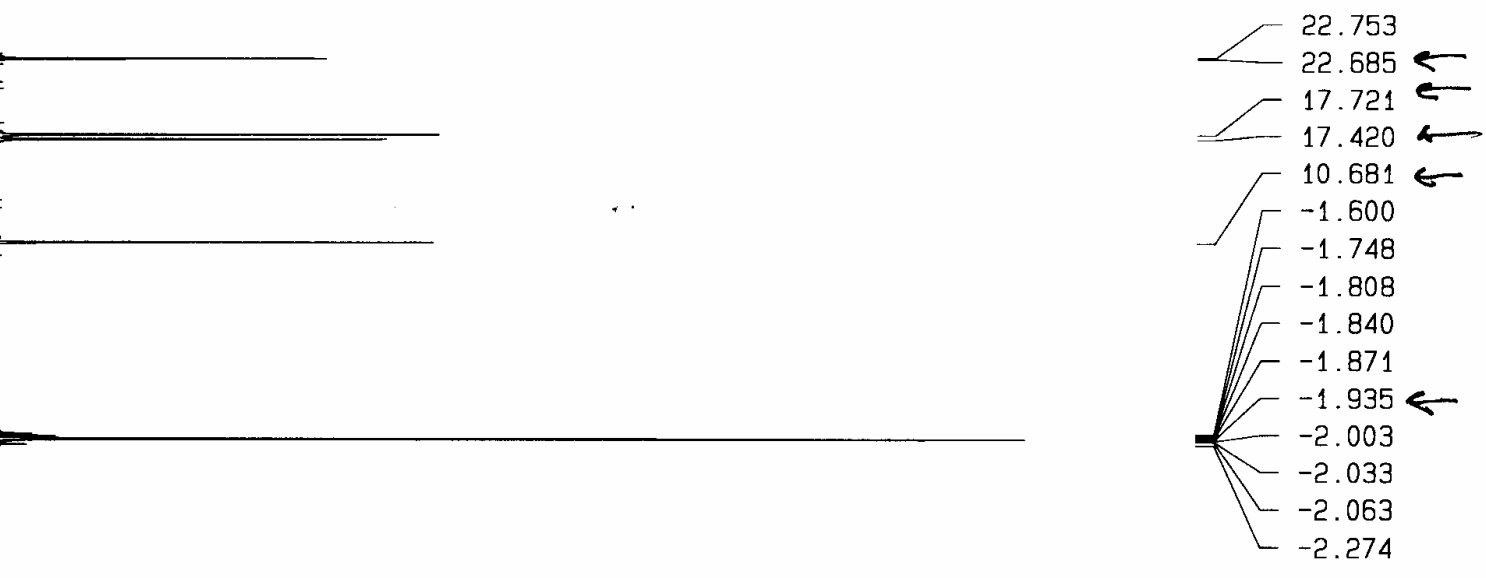


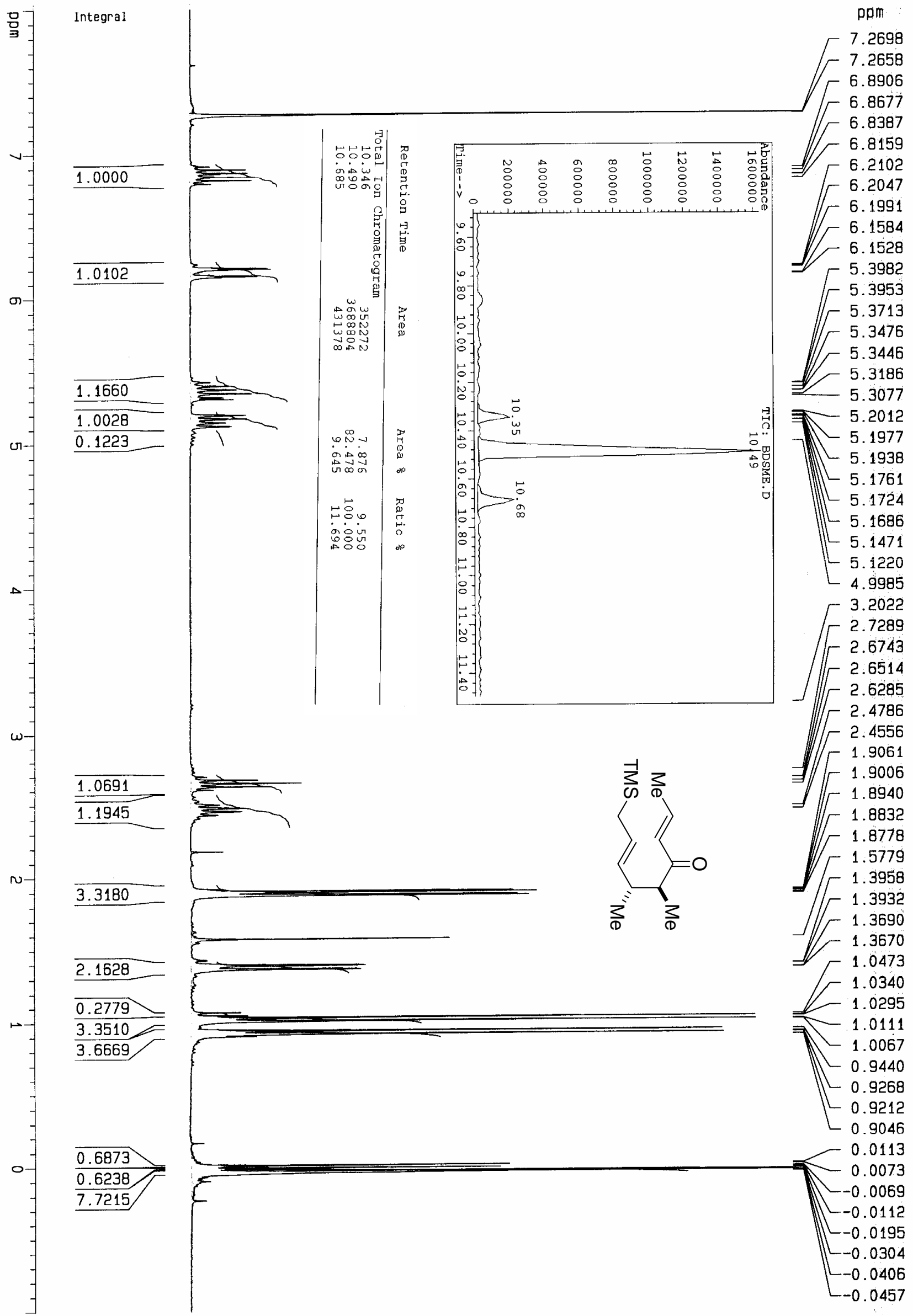



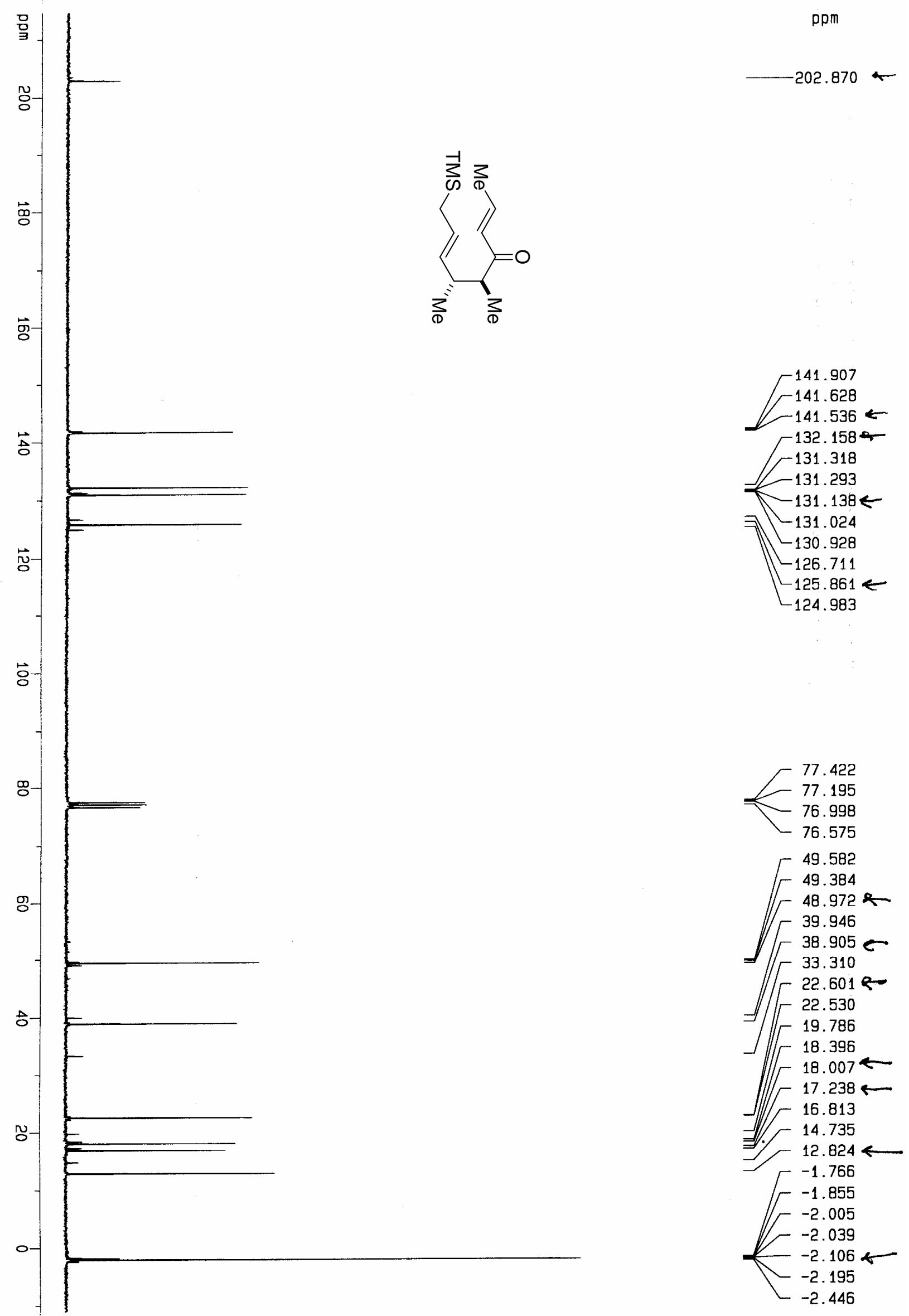


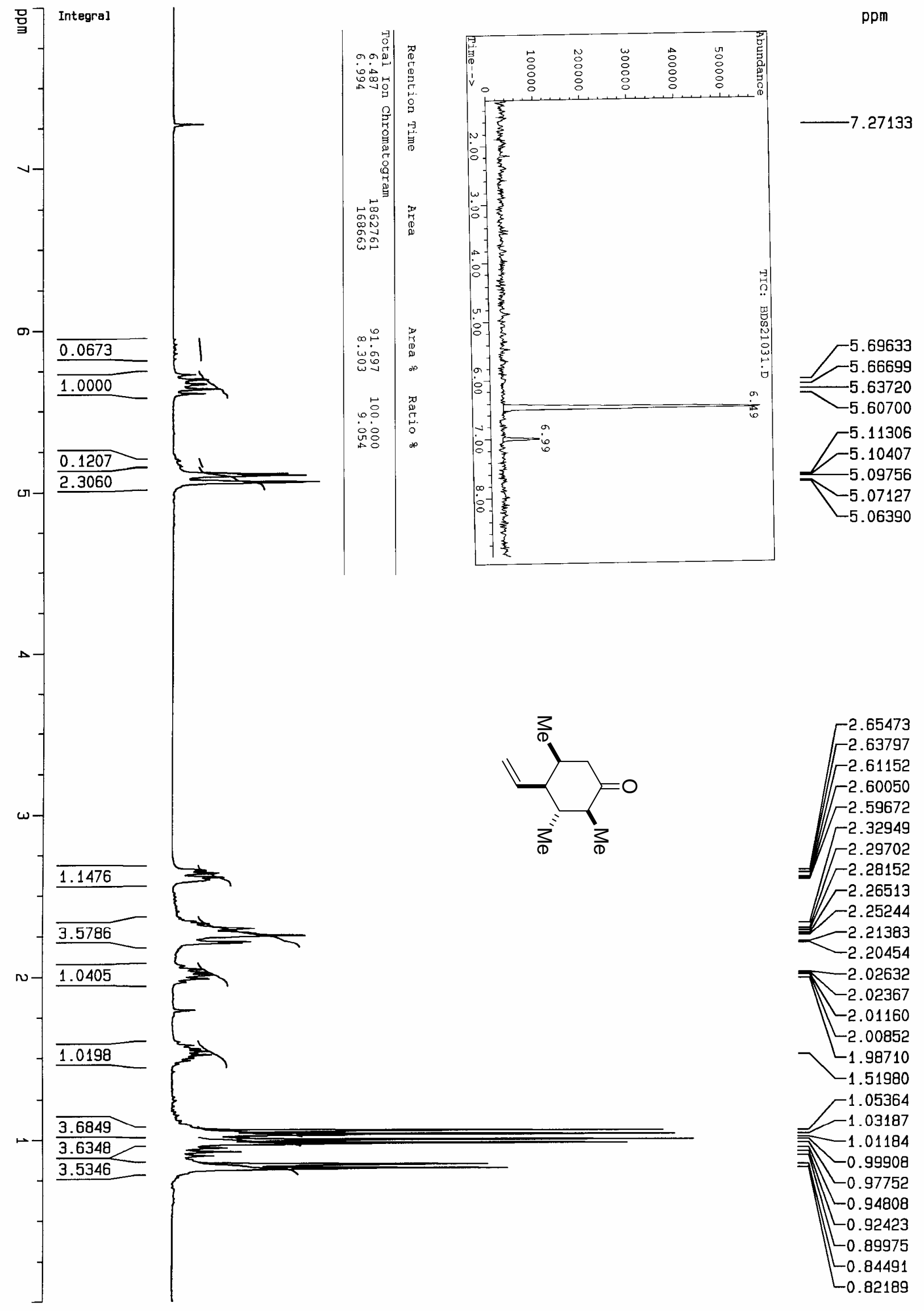



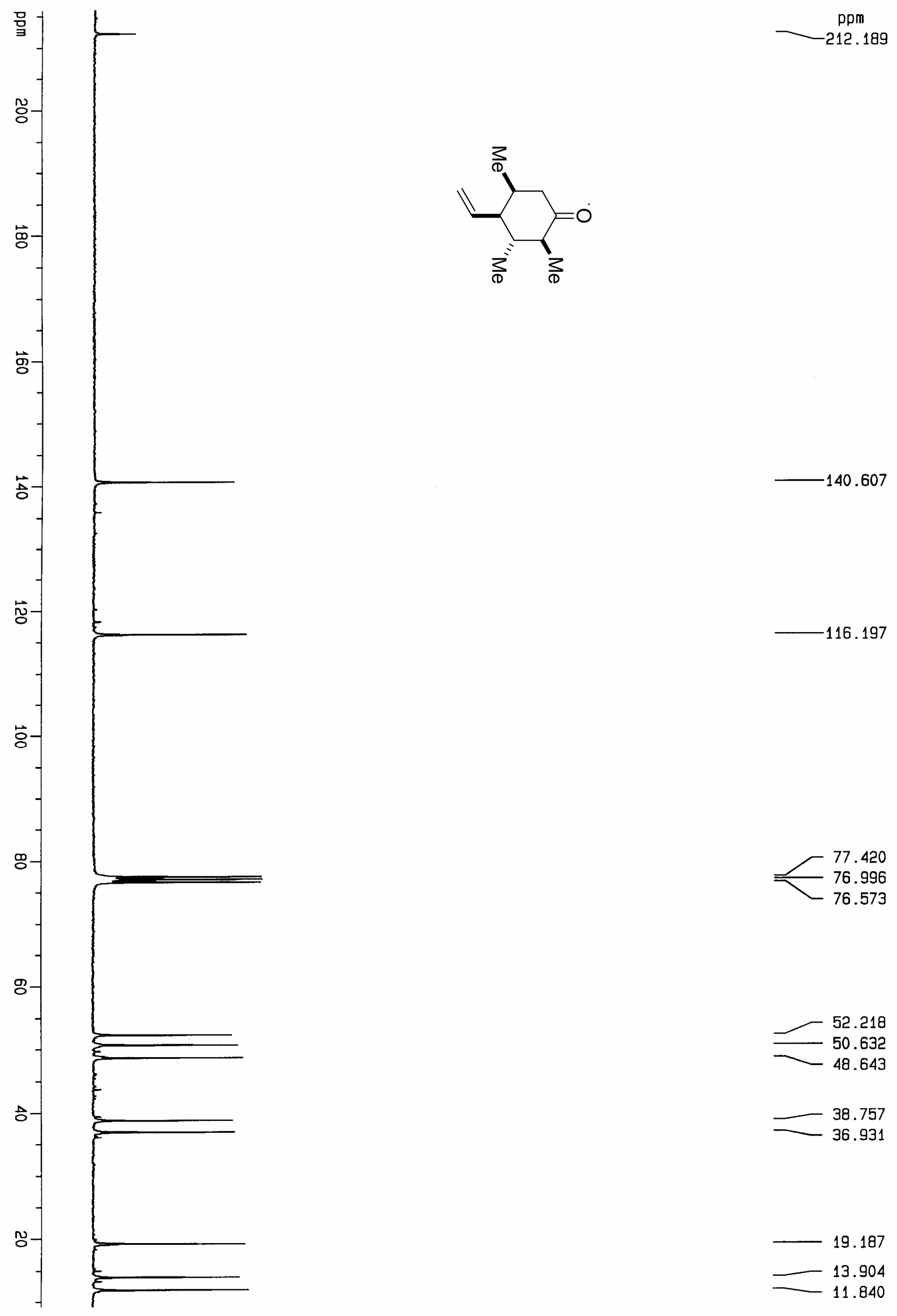


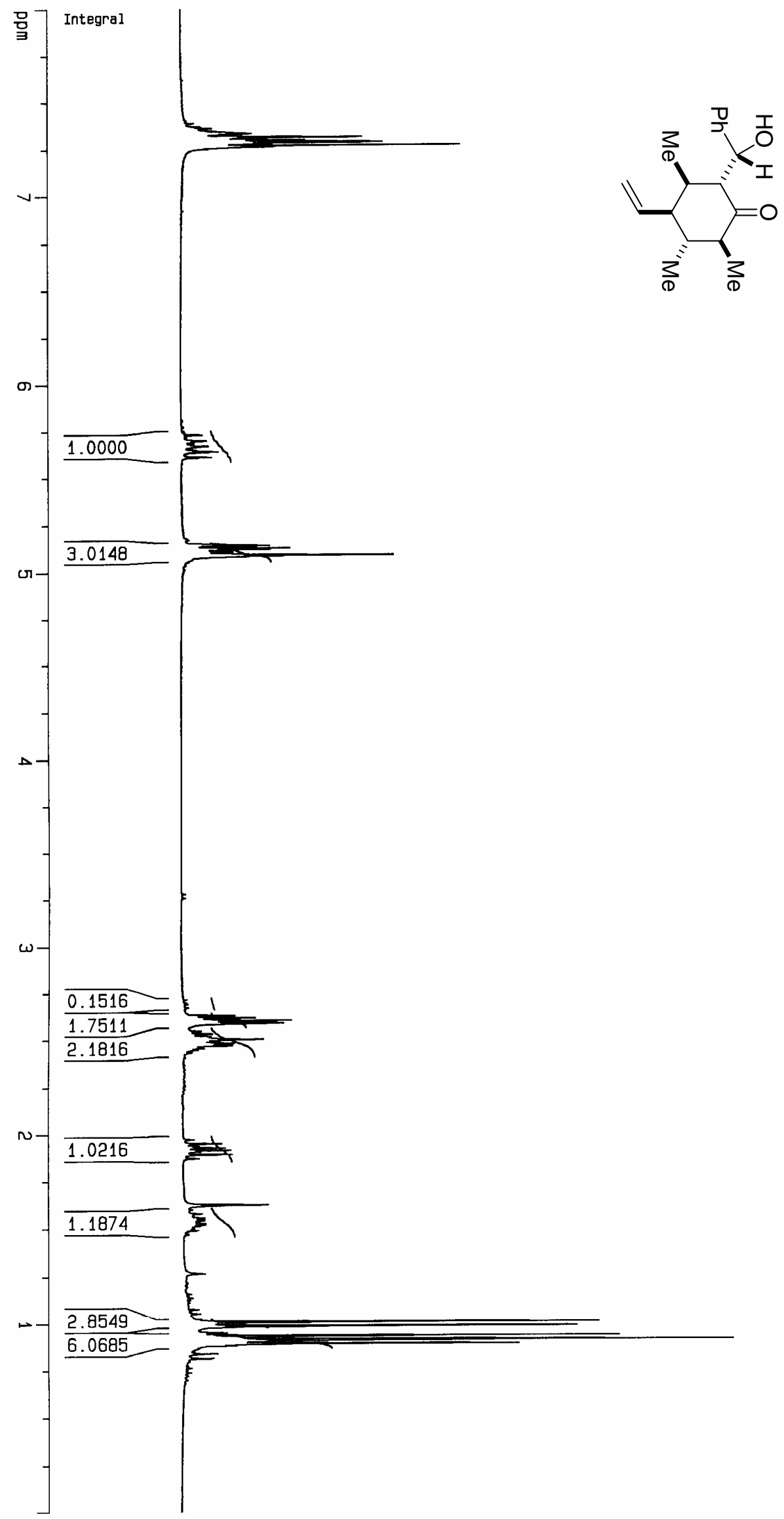

$-7.32869$

$-7.31249$

$-7.30036$

$-7.29691$

$-7.28772$

$-7.28613$

$-7.27919$

47.27598

$-7.26999$

47.26423

5.53917

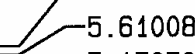

5.15070

$-5.14861$

$-5.14435$

$-5.14235$

$-5.12915$

$-5.12288$

$-5.10774$

$-5.09659$

$-5.09058$

ᄂ5.08347

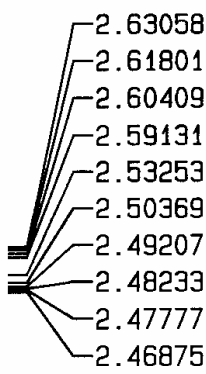

$-1.94922$

$-1.92763$

$-1.91404$

$-1.89246$

$-1.62323$

1.00777

0.98612

0.95082

$-0.93321$

$-0.92156$

$=0.91430$

$-0.91178$

$-0.90284$

Lo.89120

Lo.87904

Lo.83760

Lo.81320 

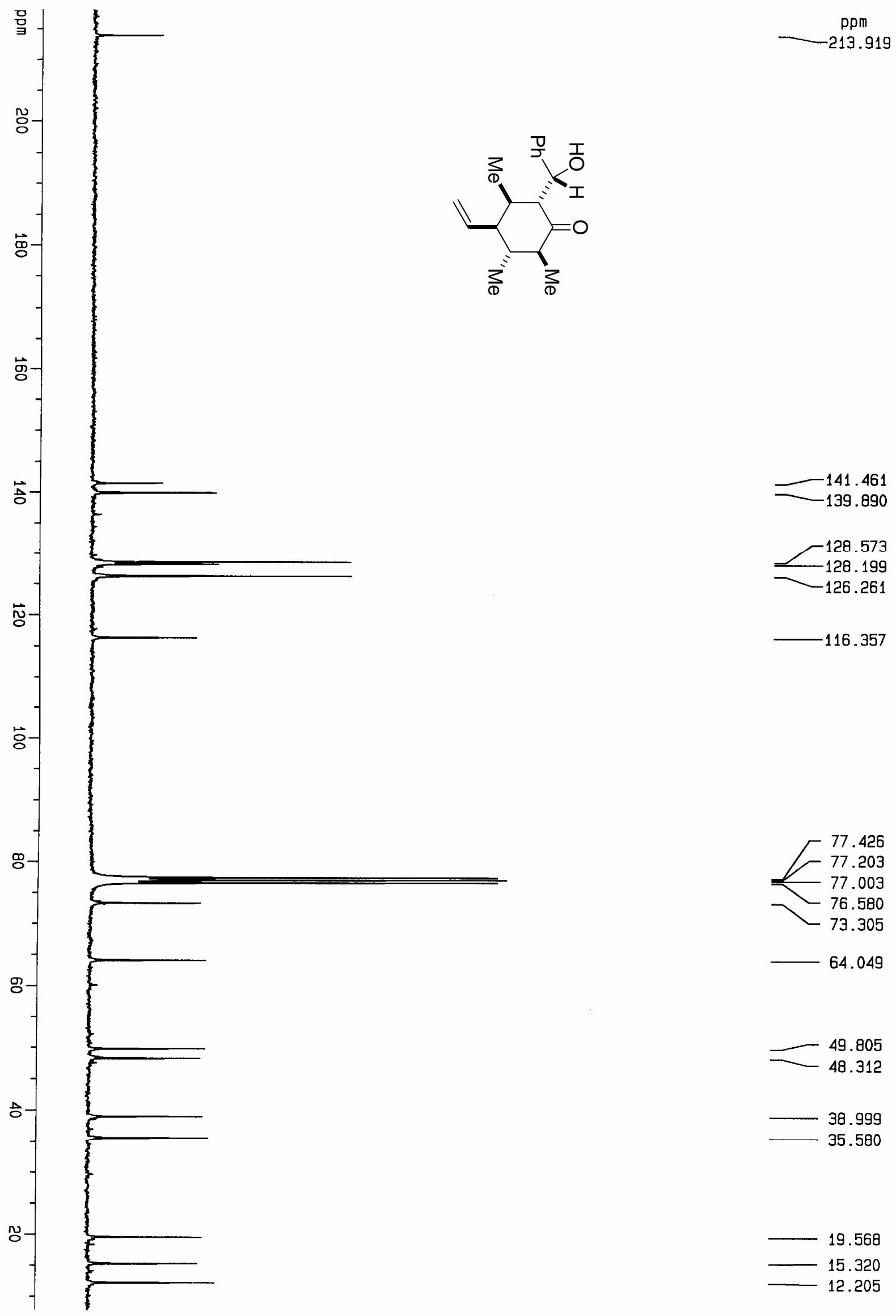


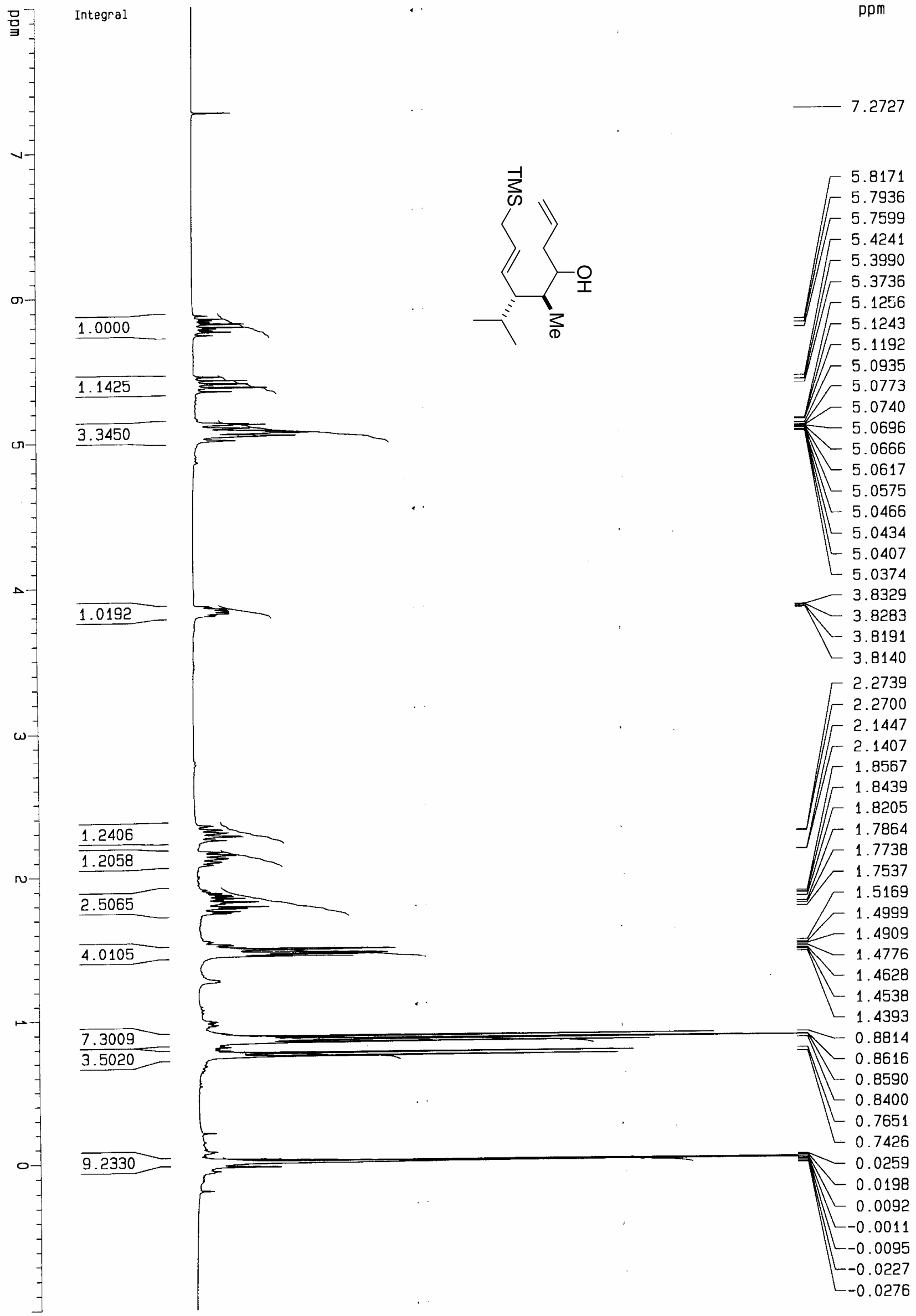




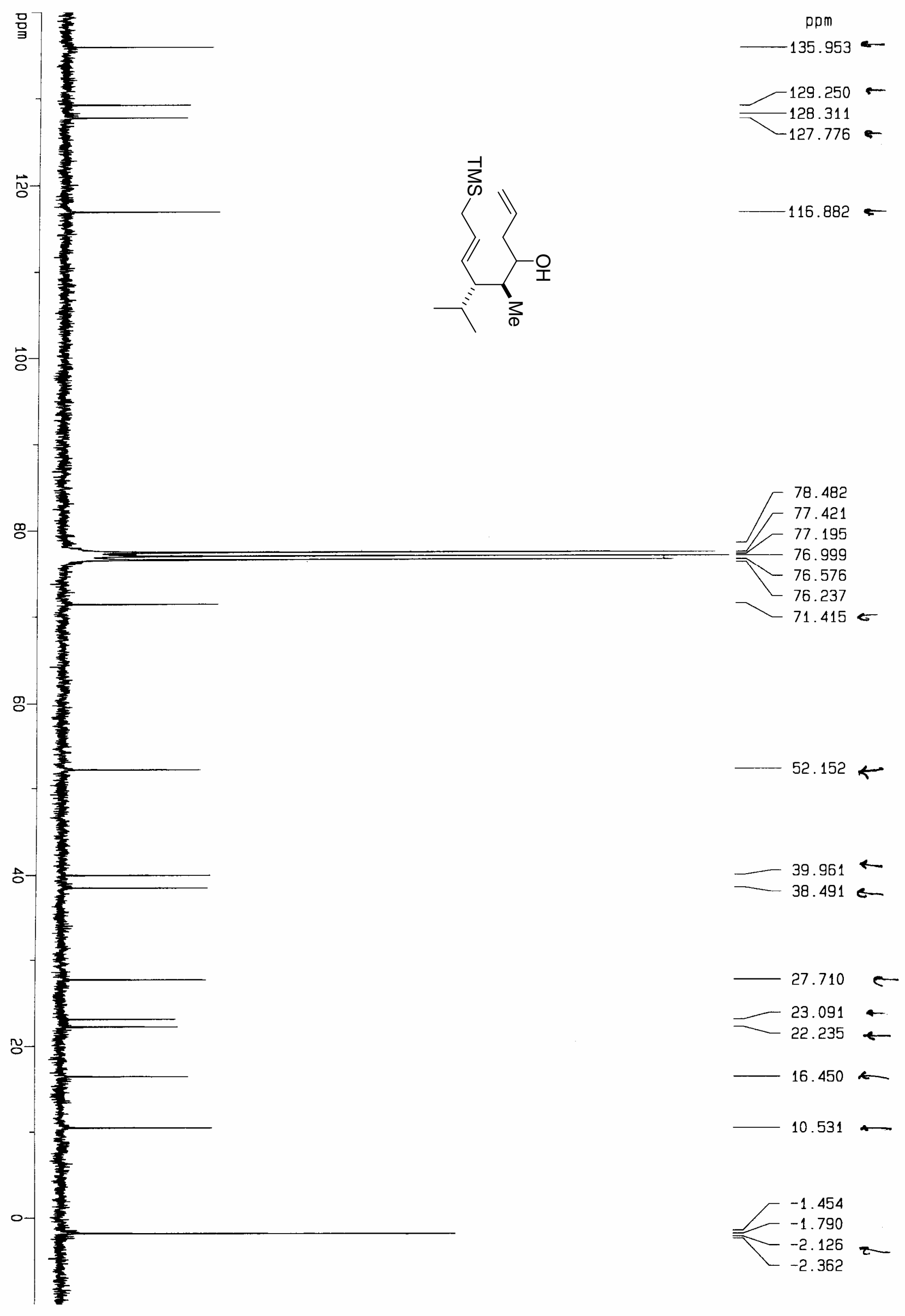




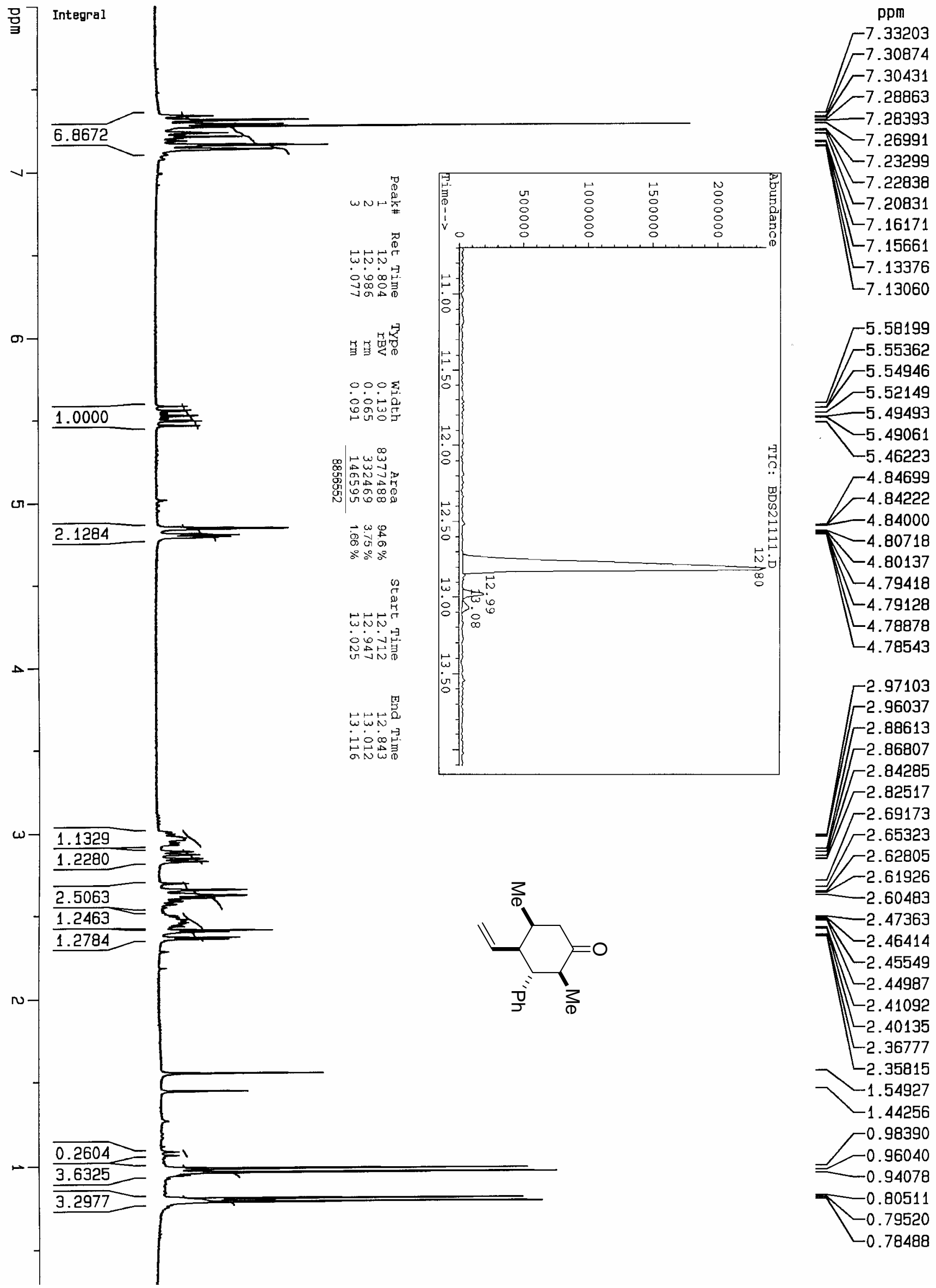




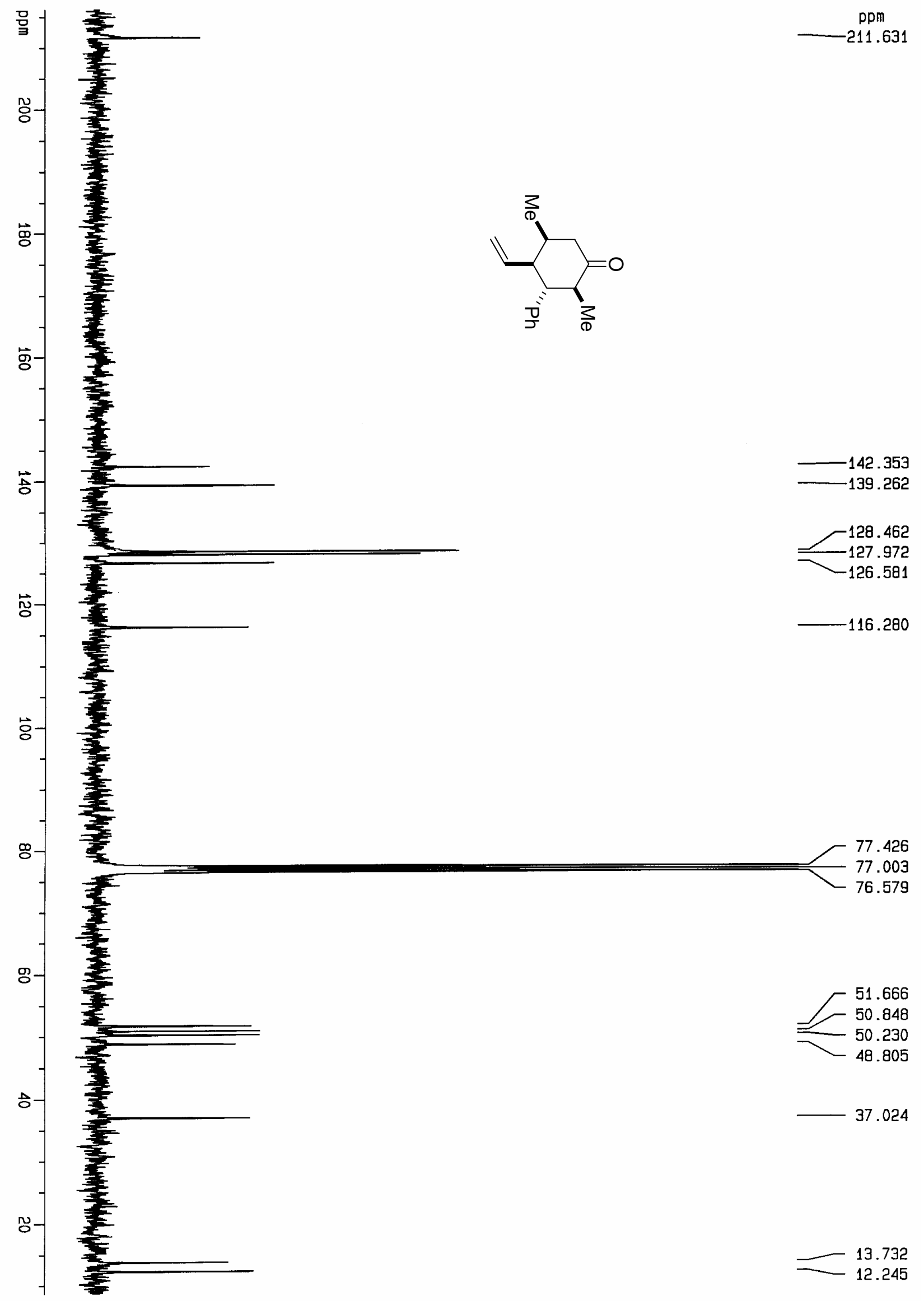




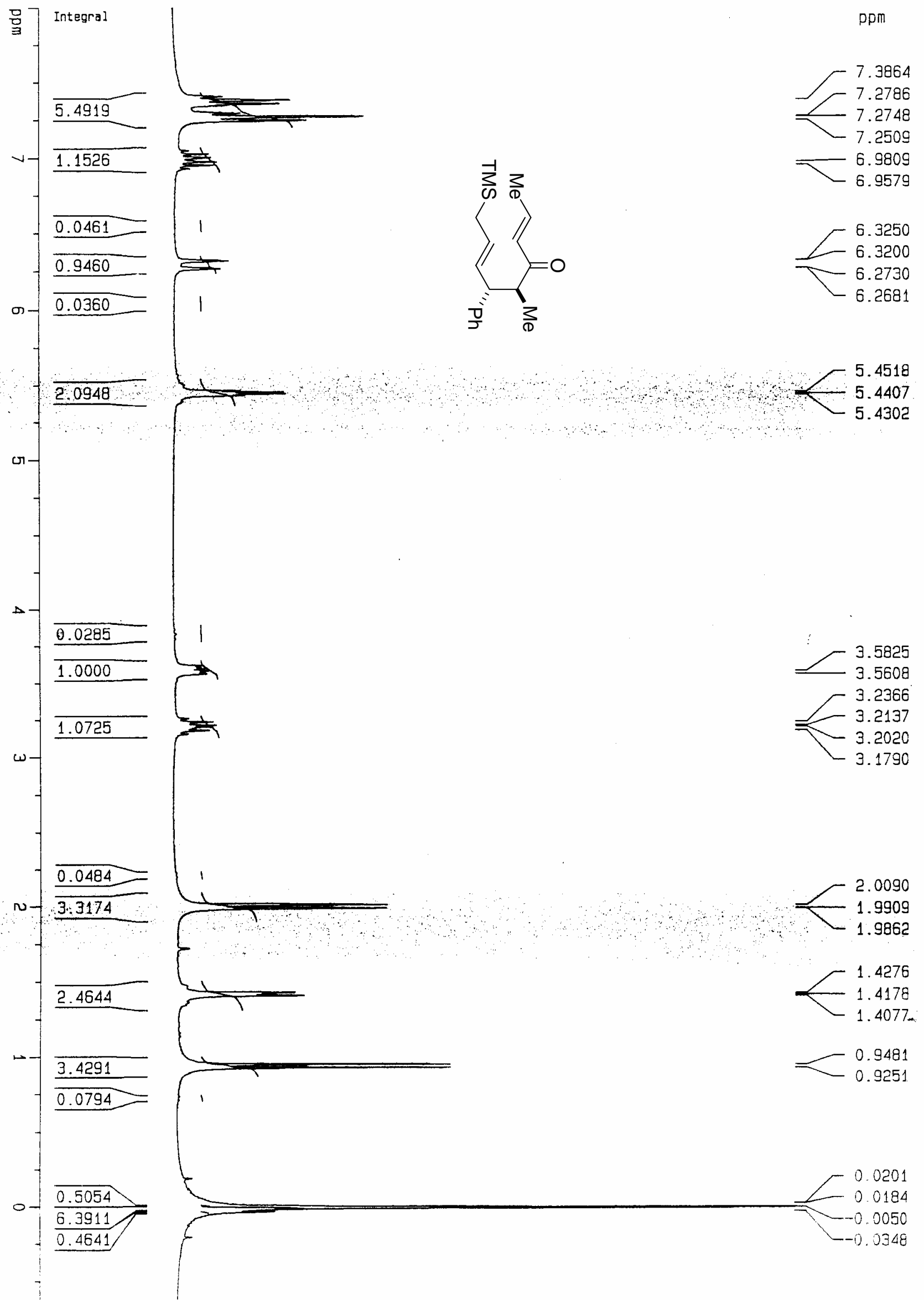



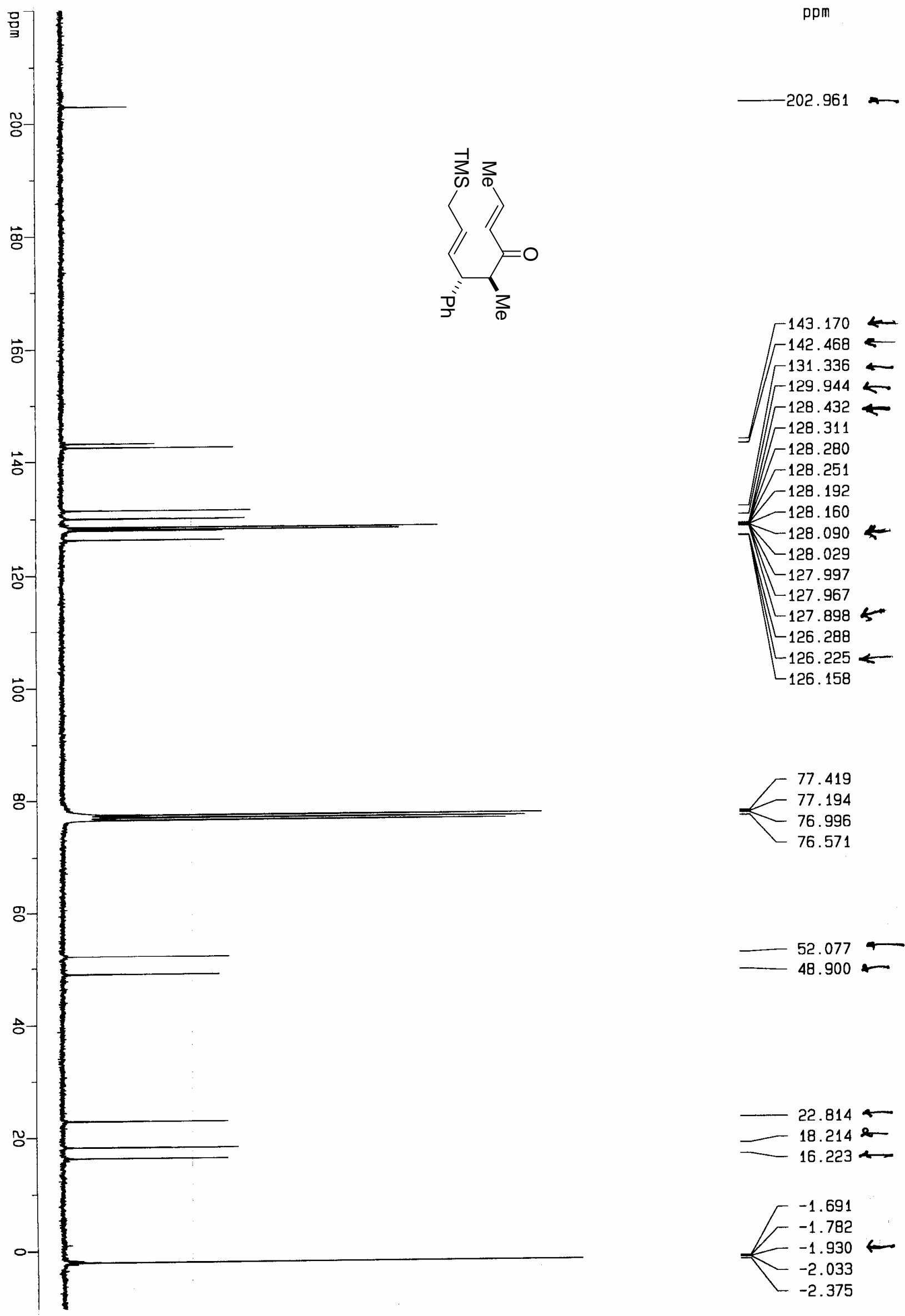


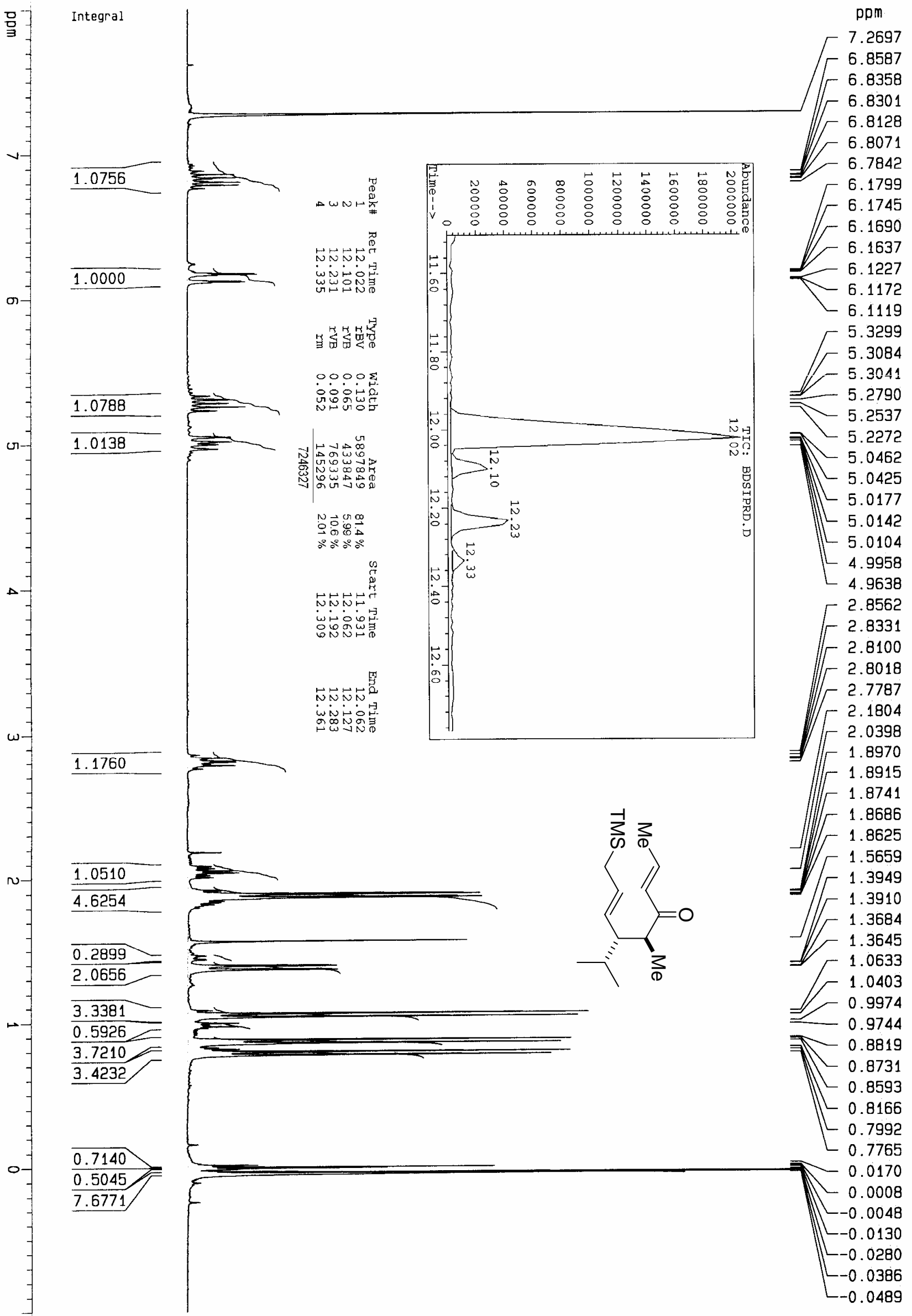



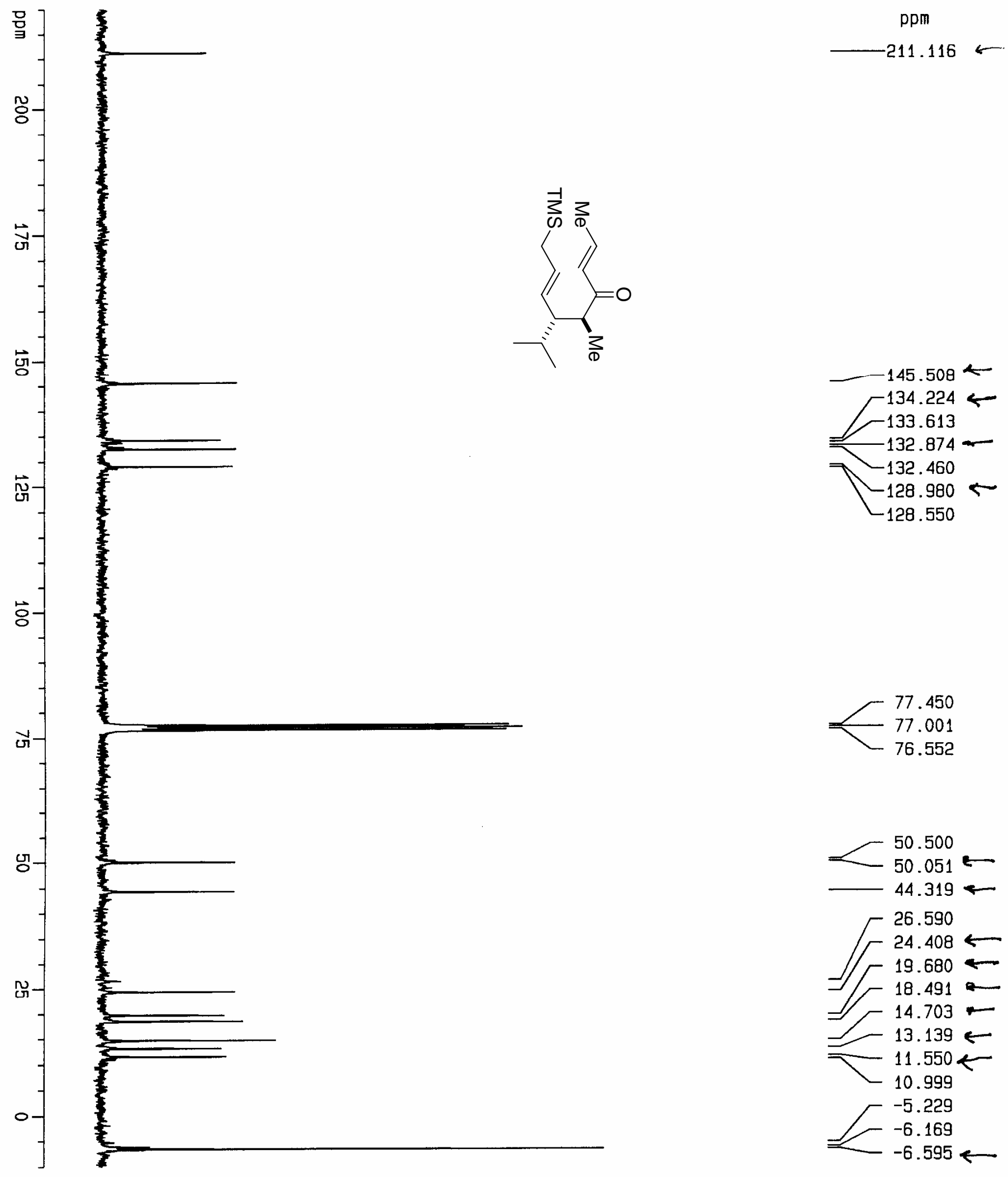


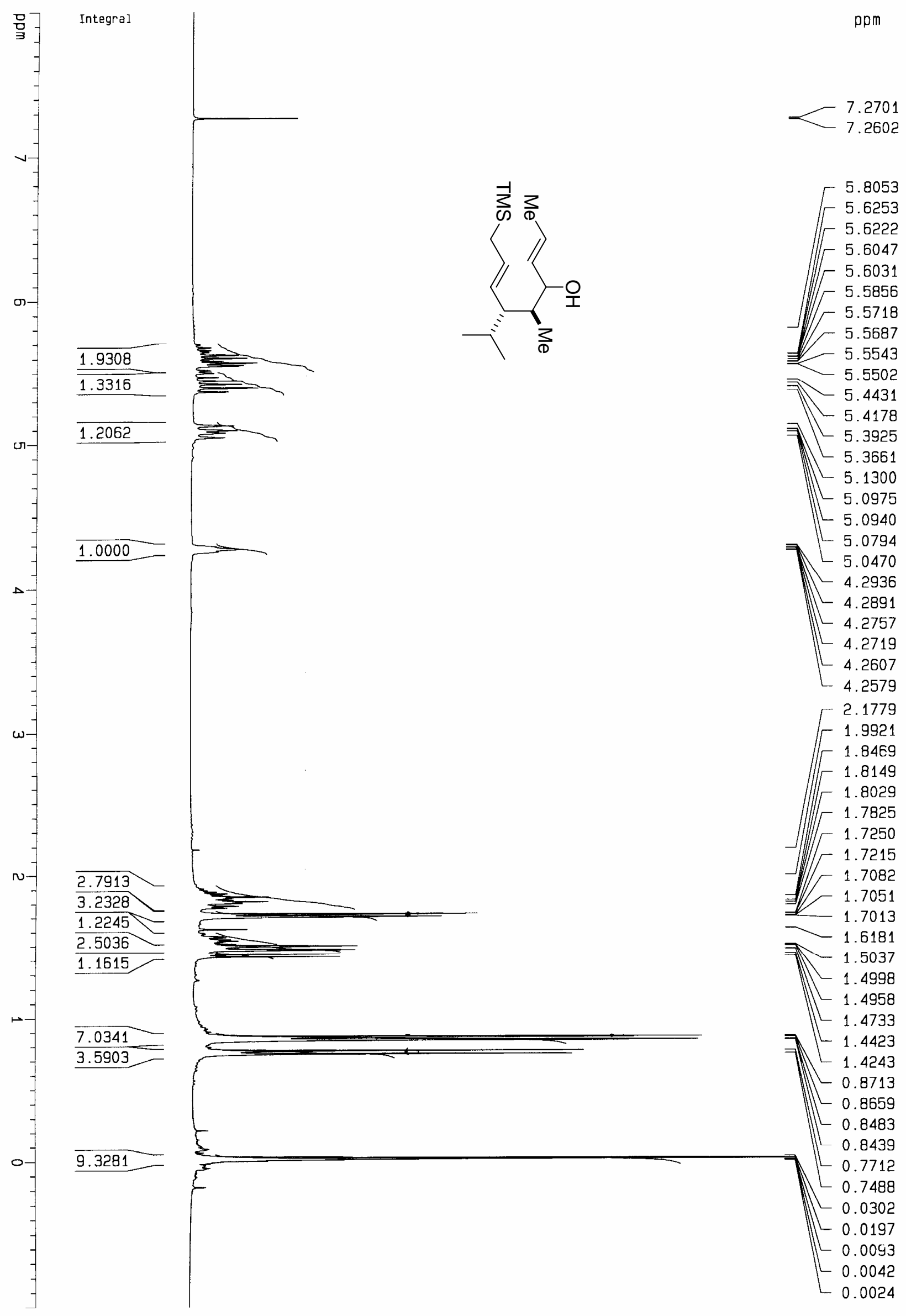



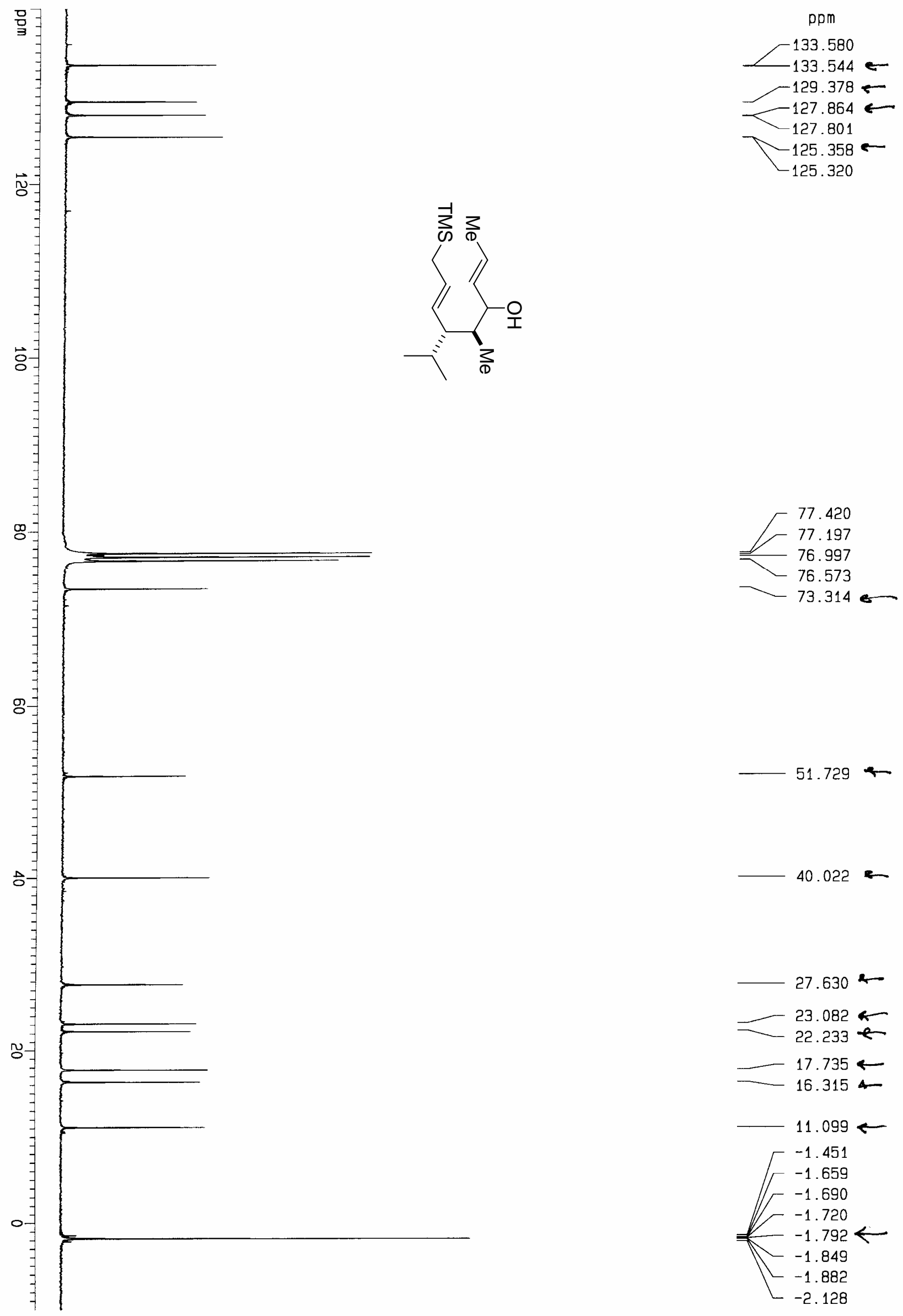


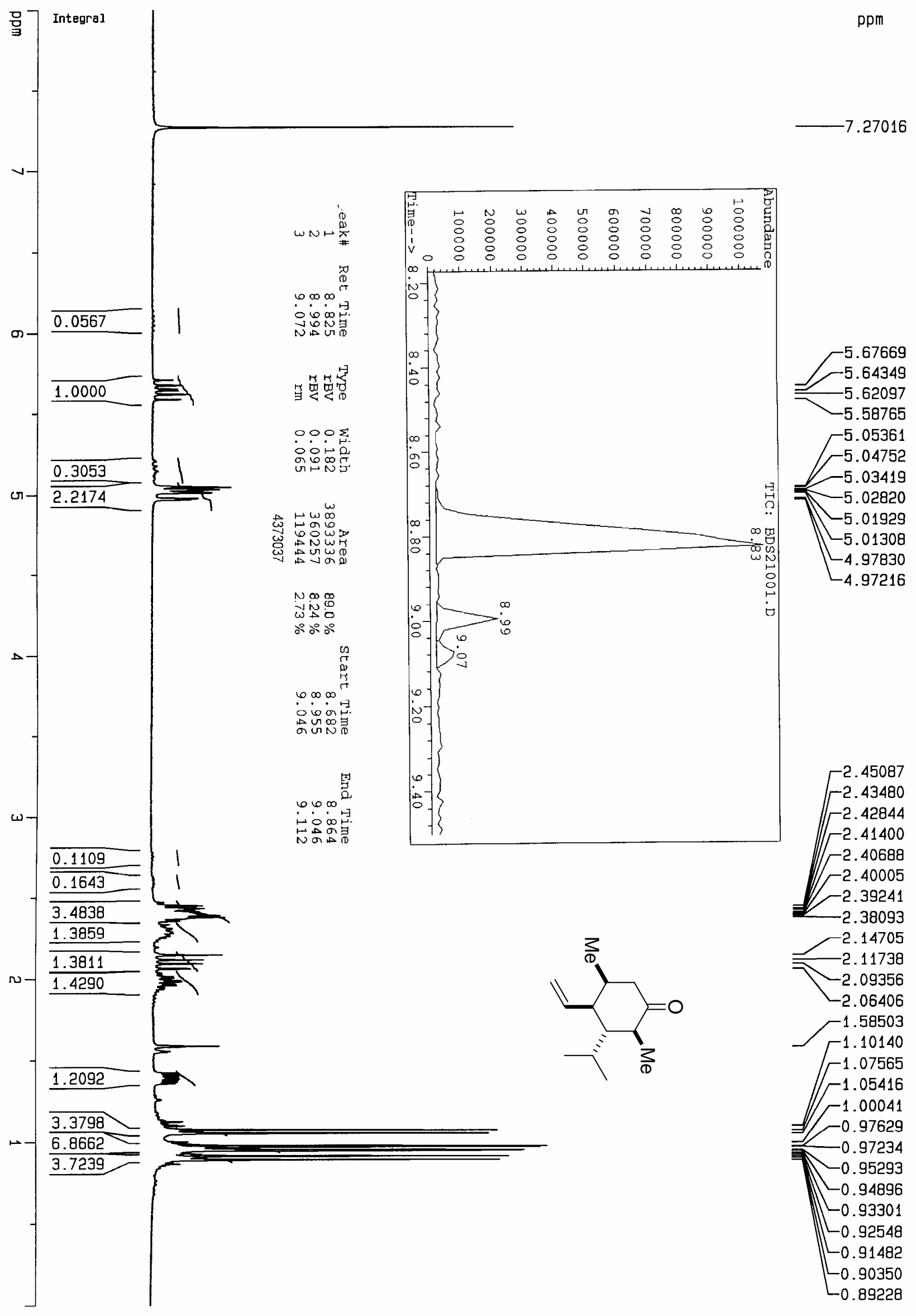



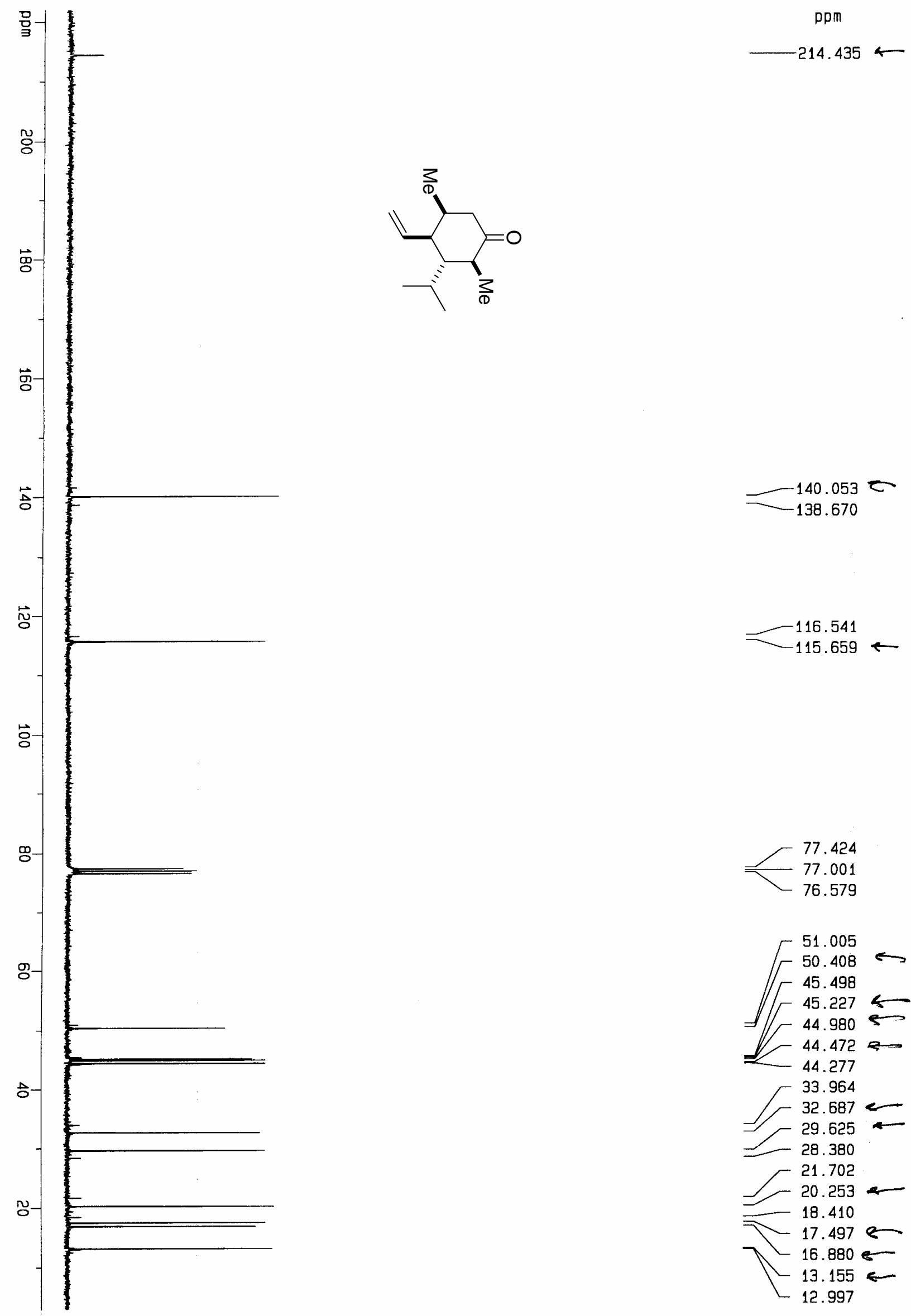


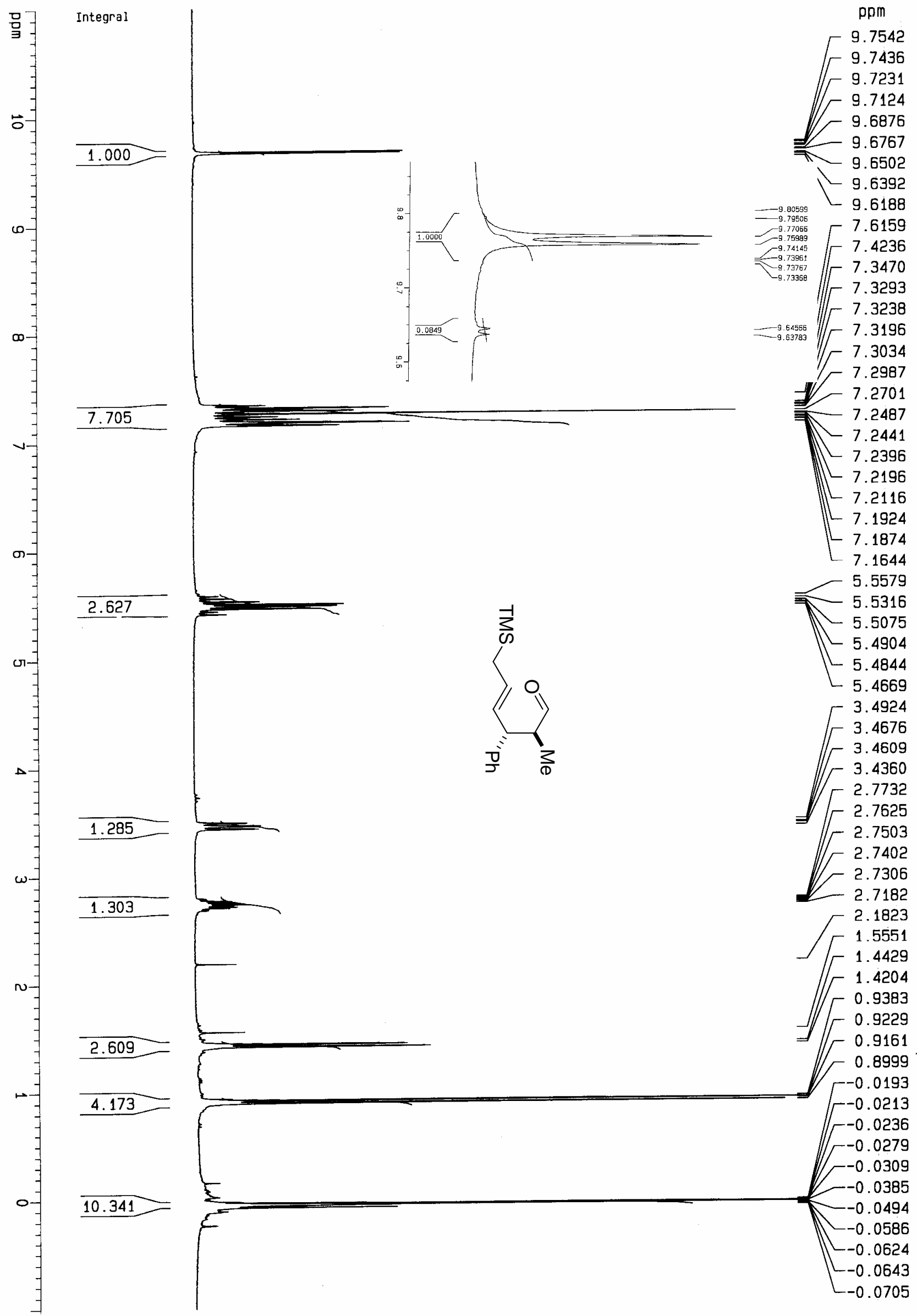



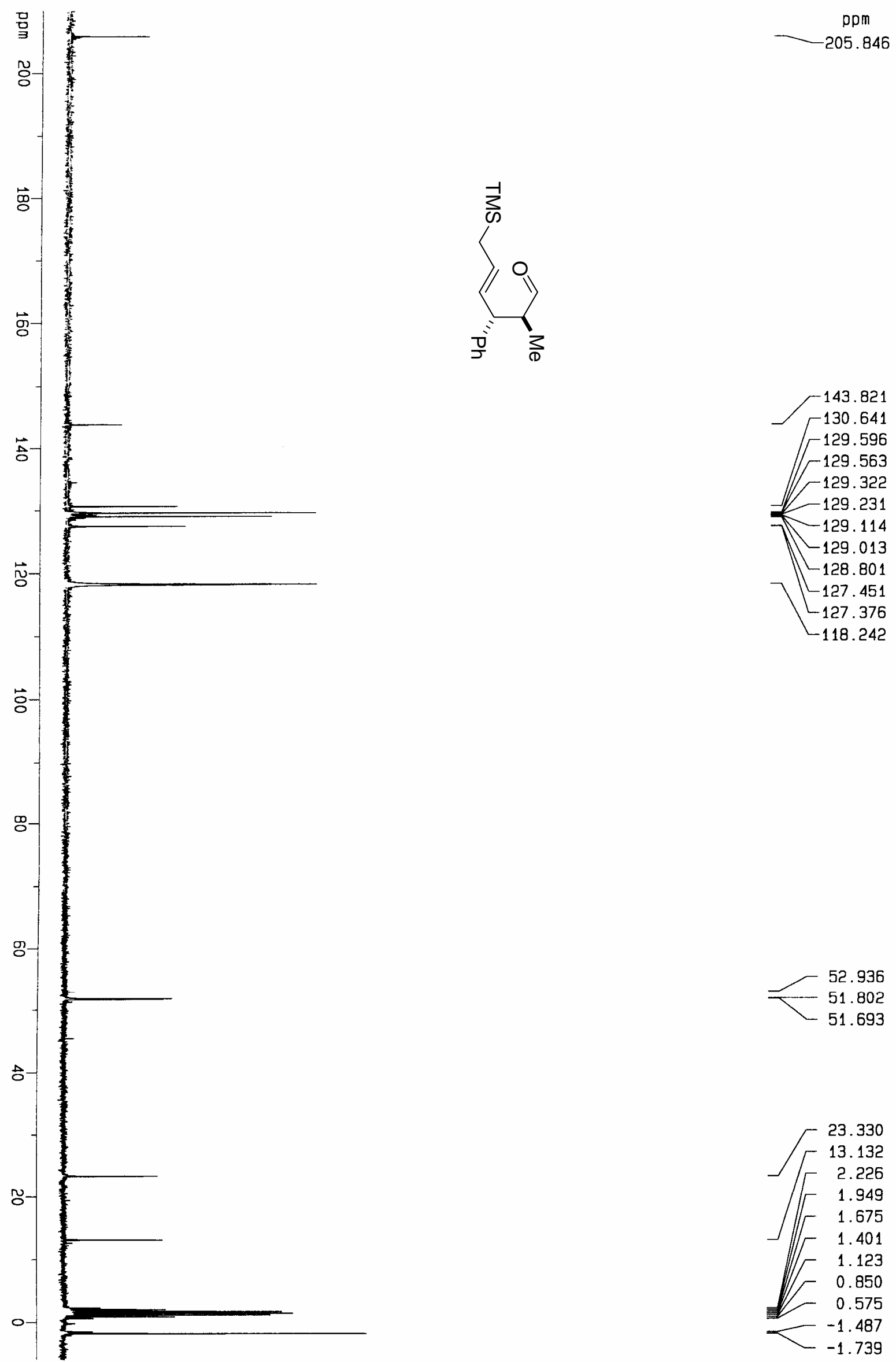

-S 54 - 


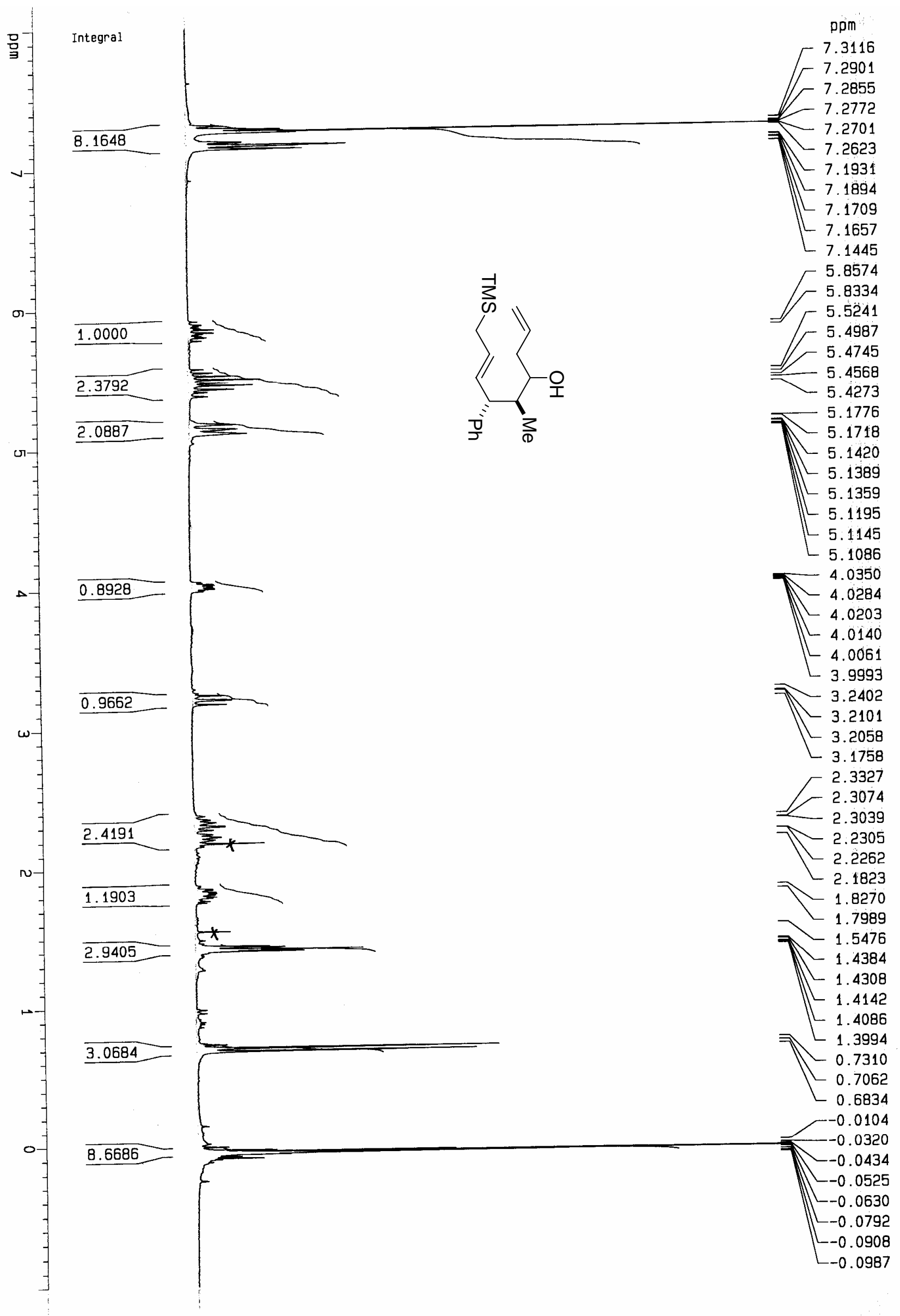




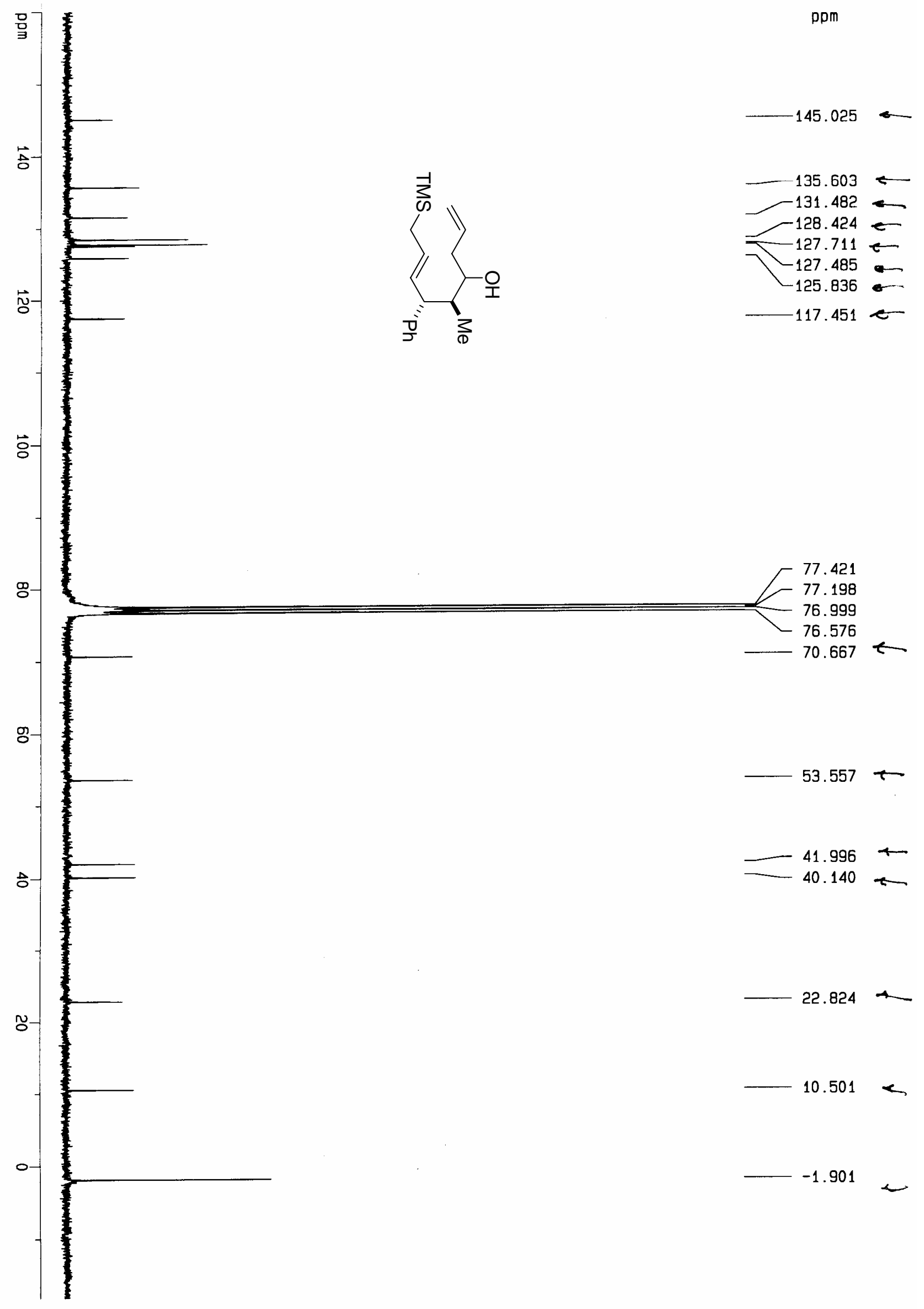




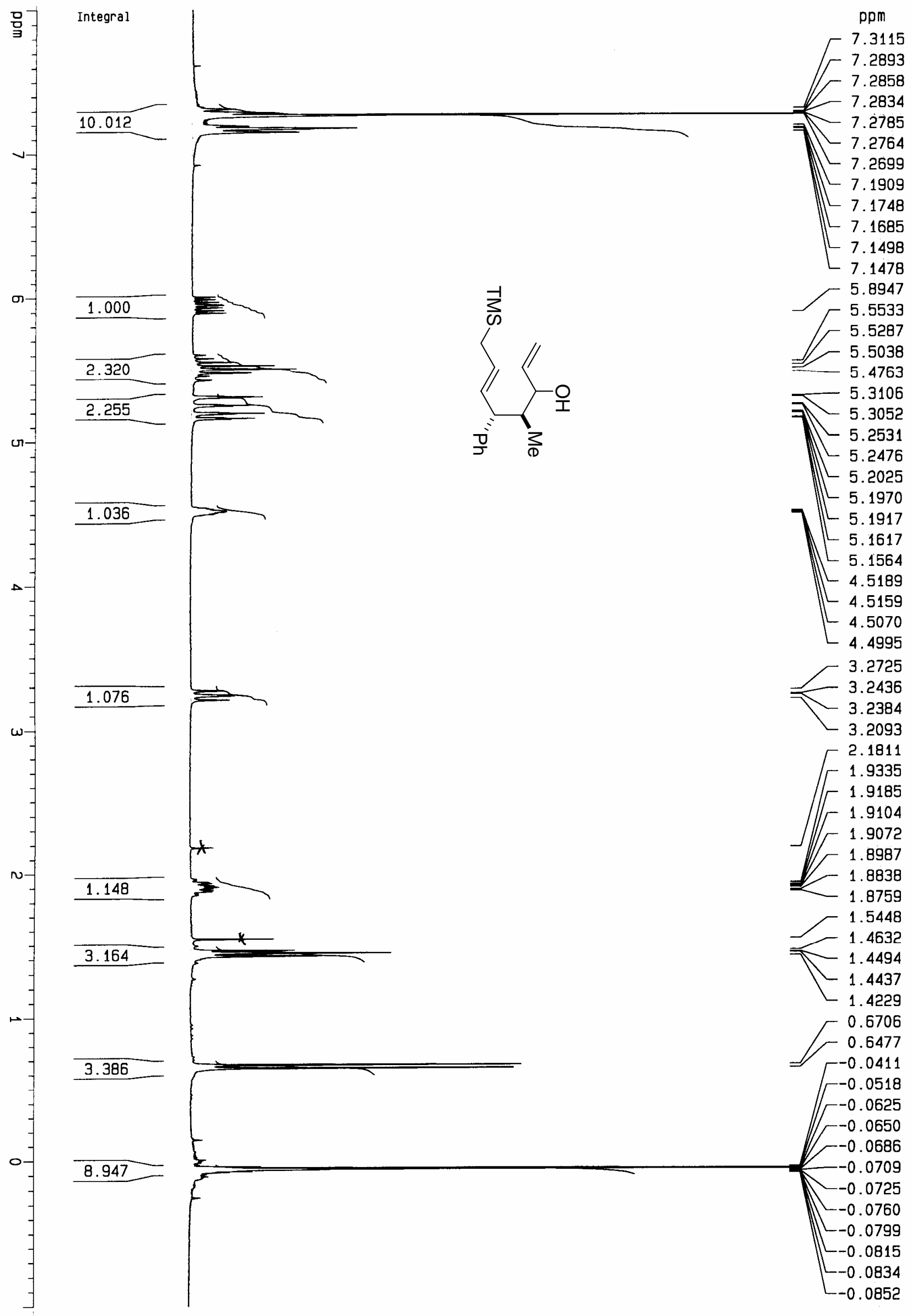



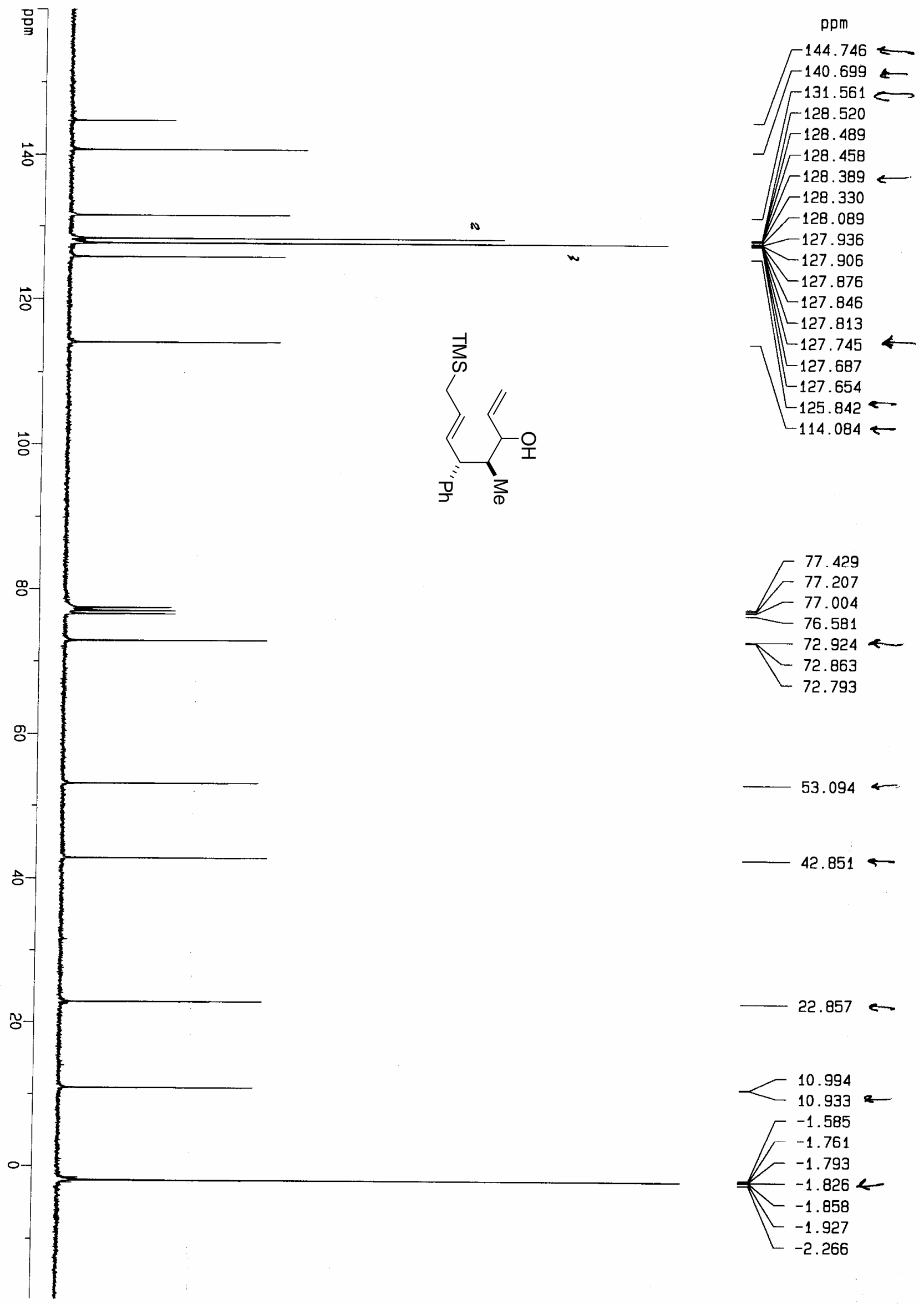


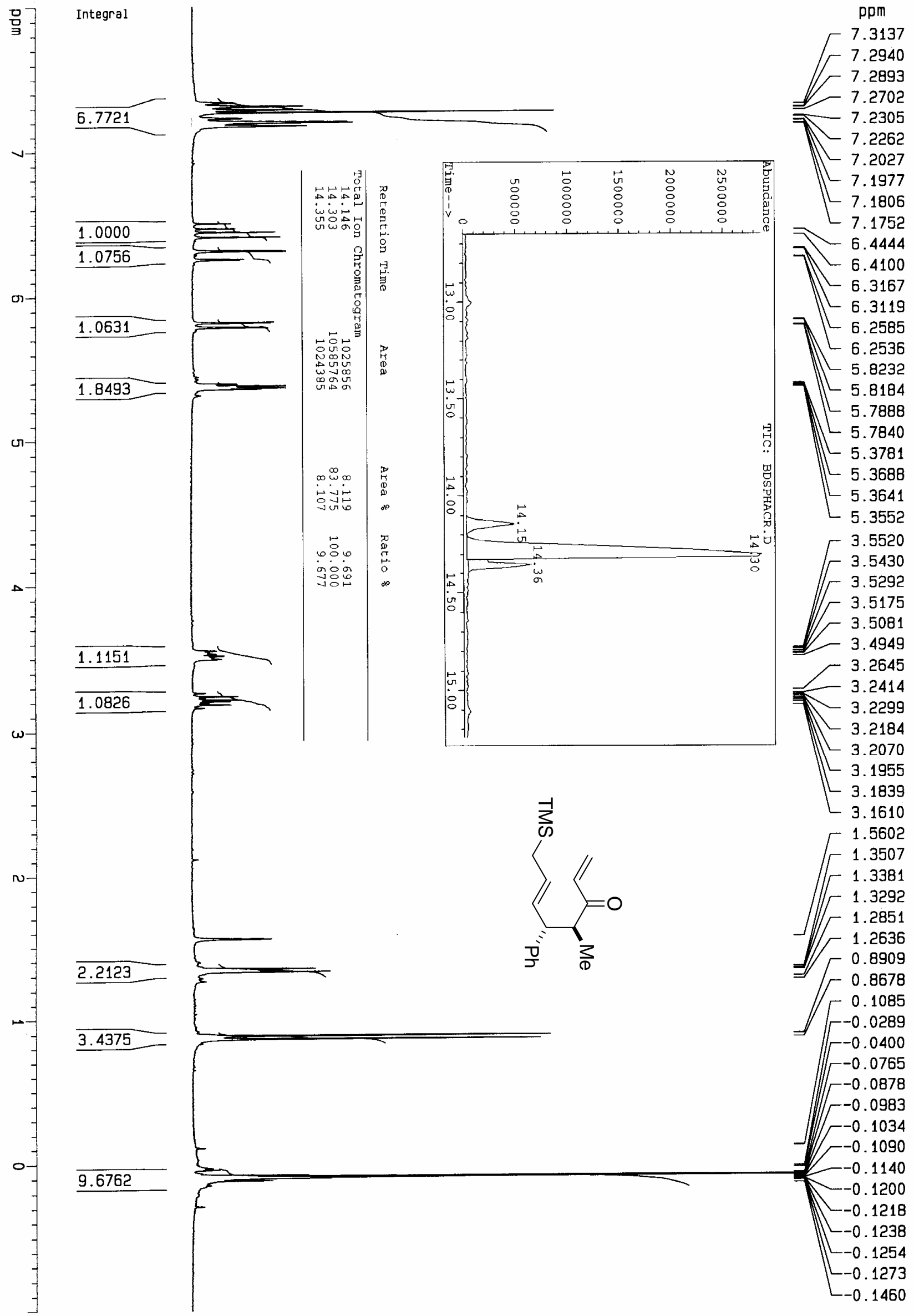




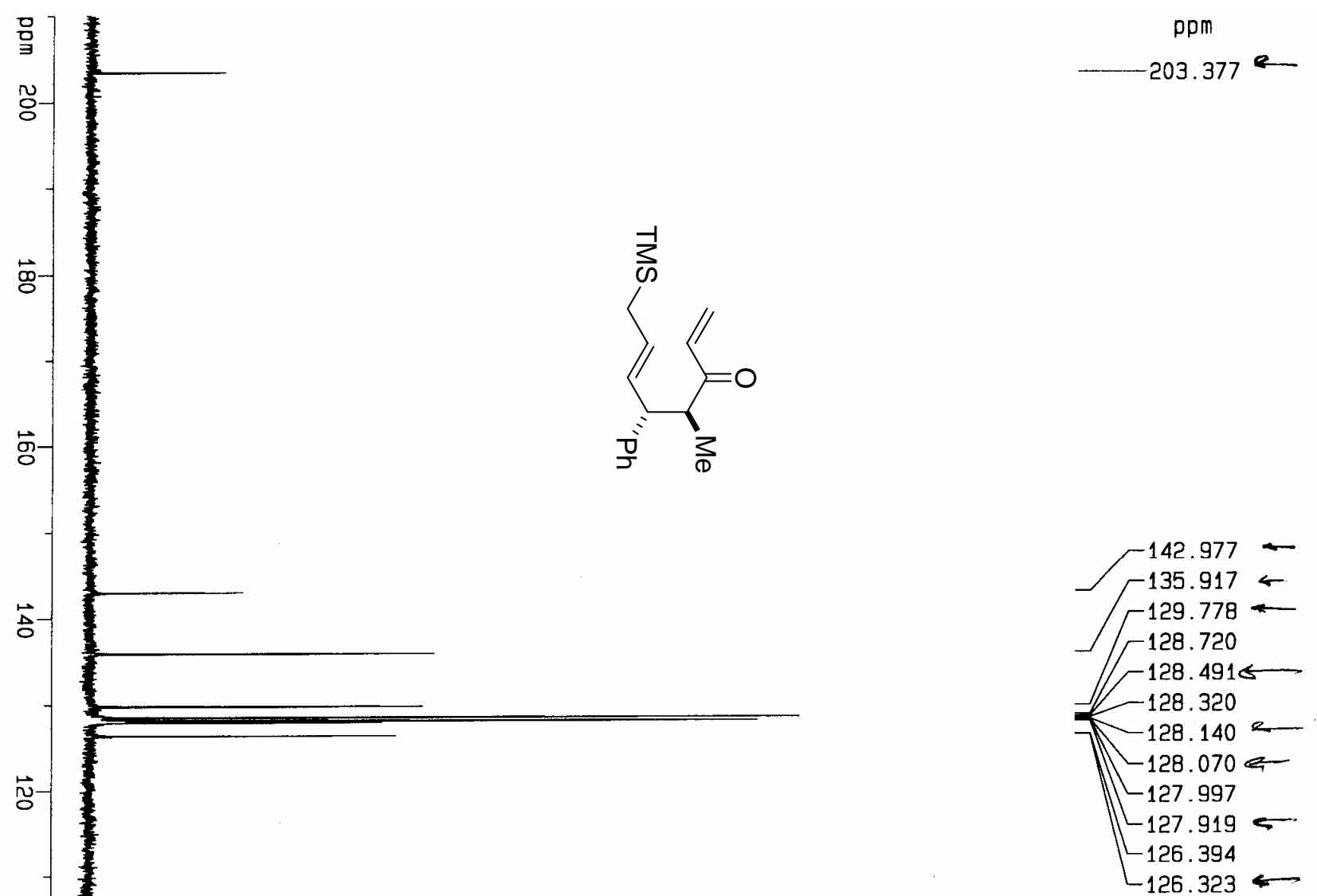

후

。

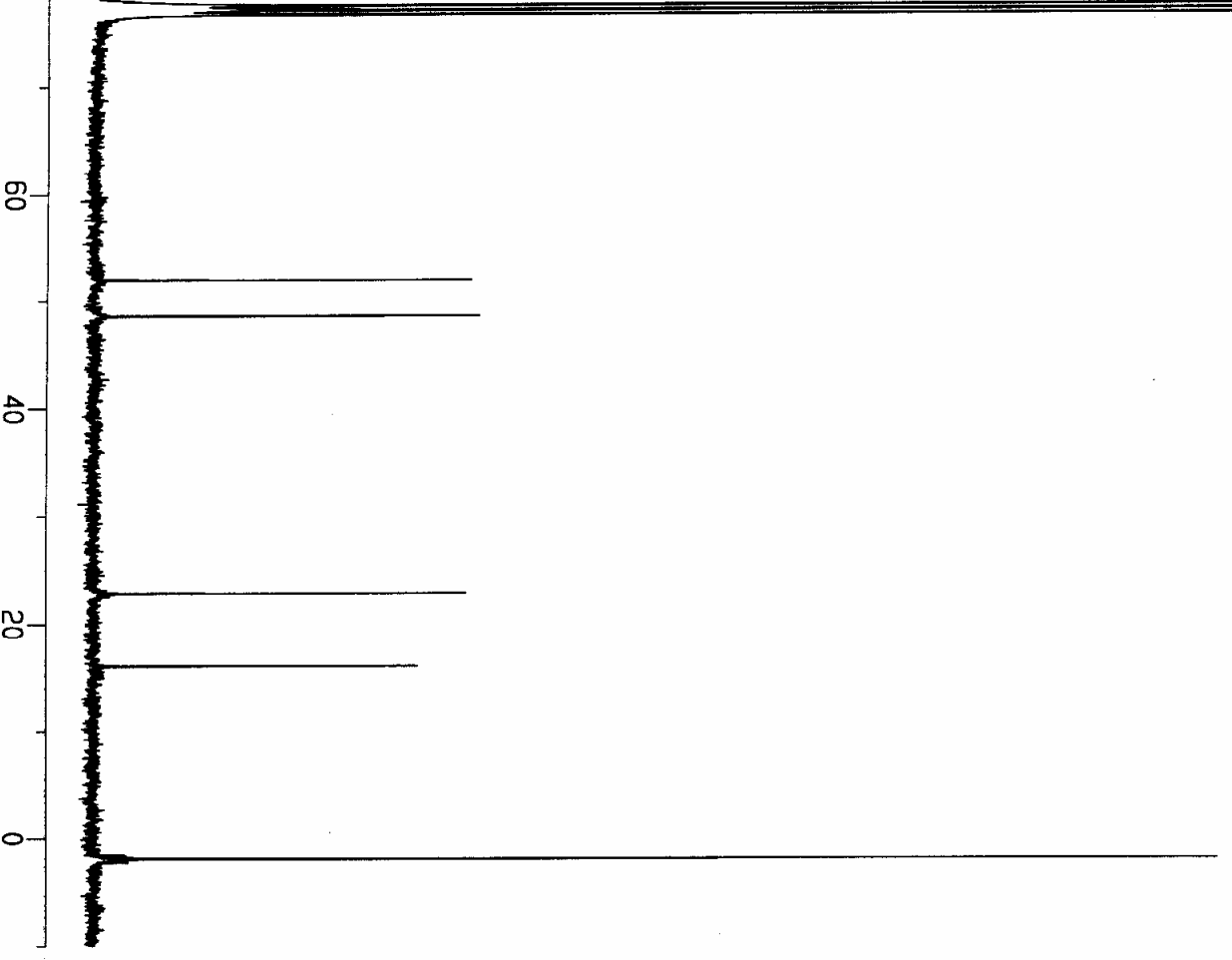

78.067

77.424

77.197

77.001

76.579

52.0206 $48.542 \leftarrow$

-S 60 - 


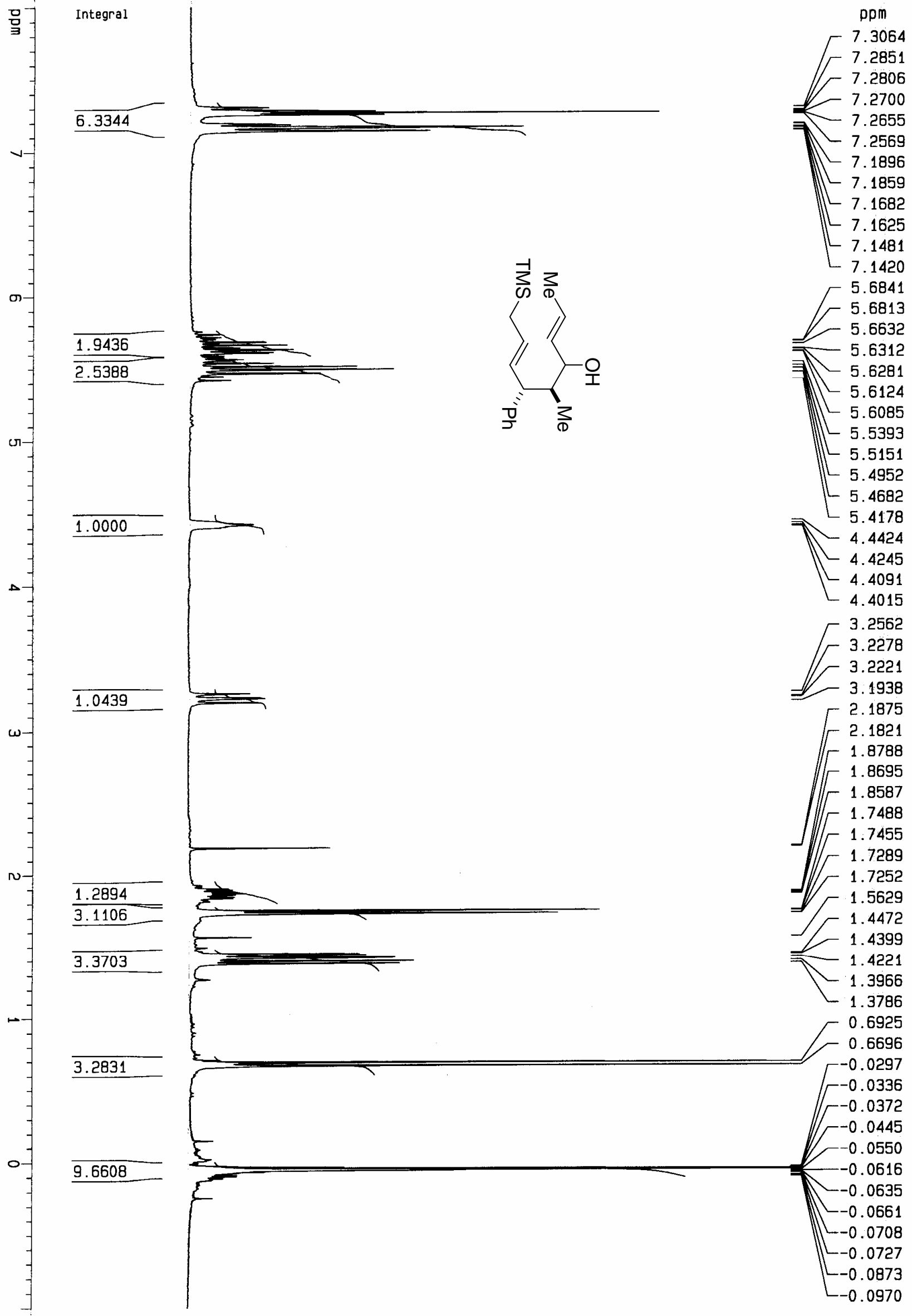



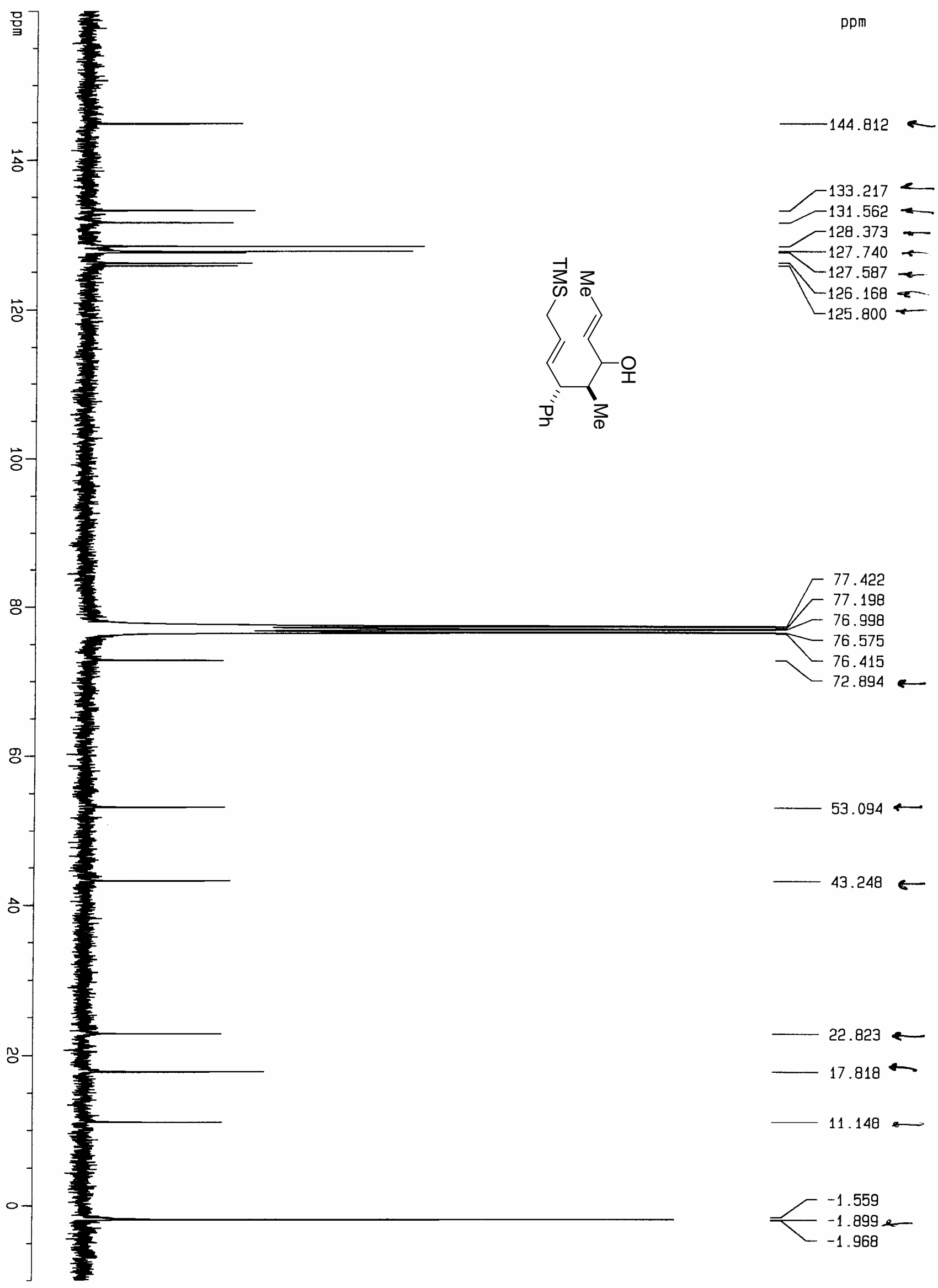


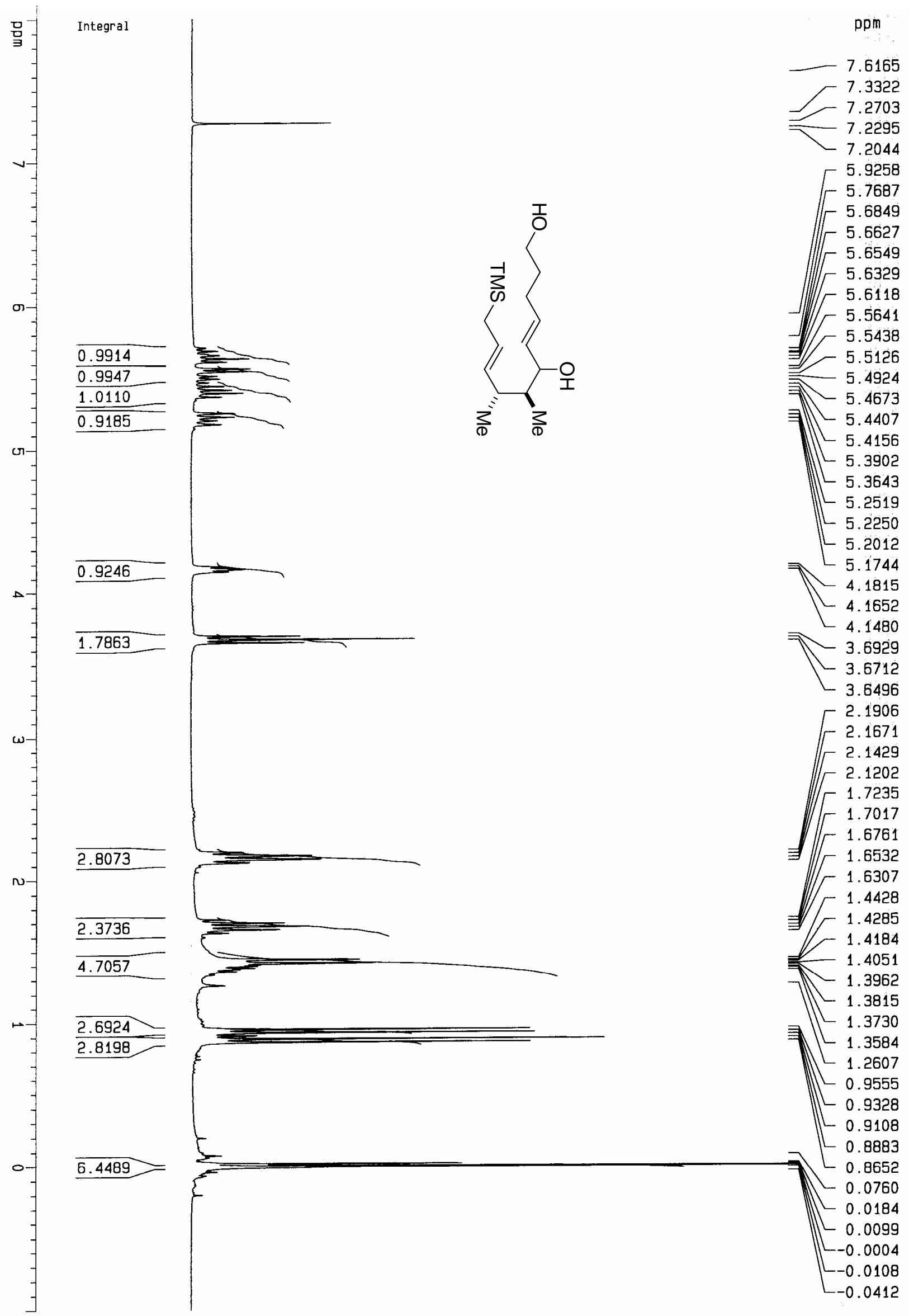



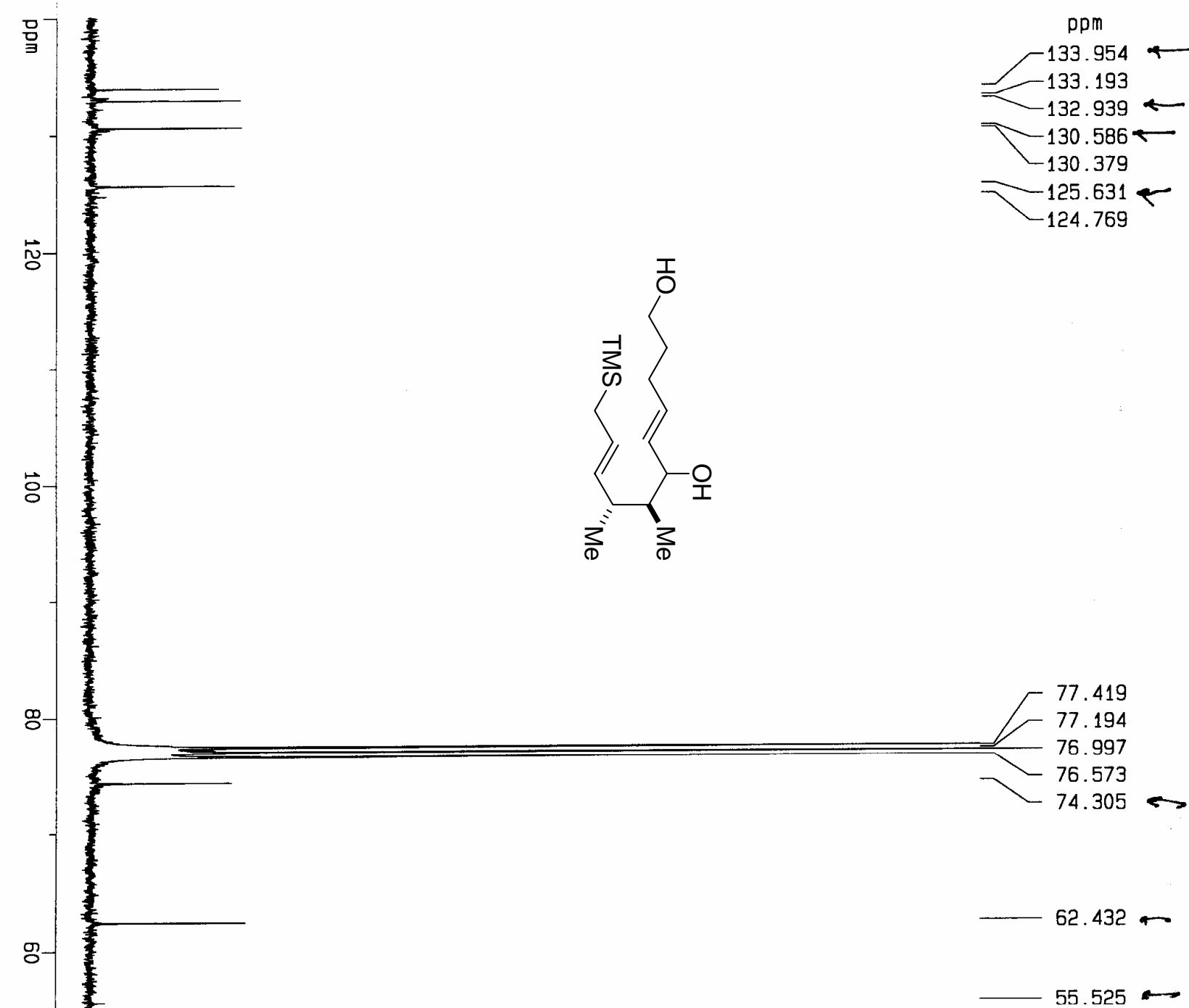

o

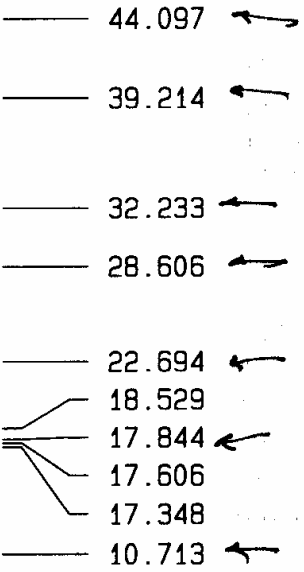

o-

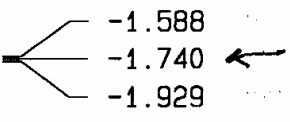




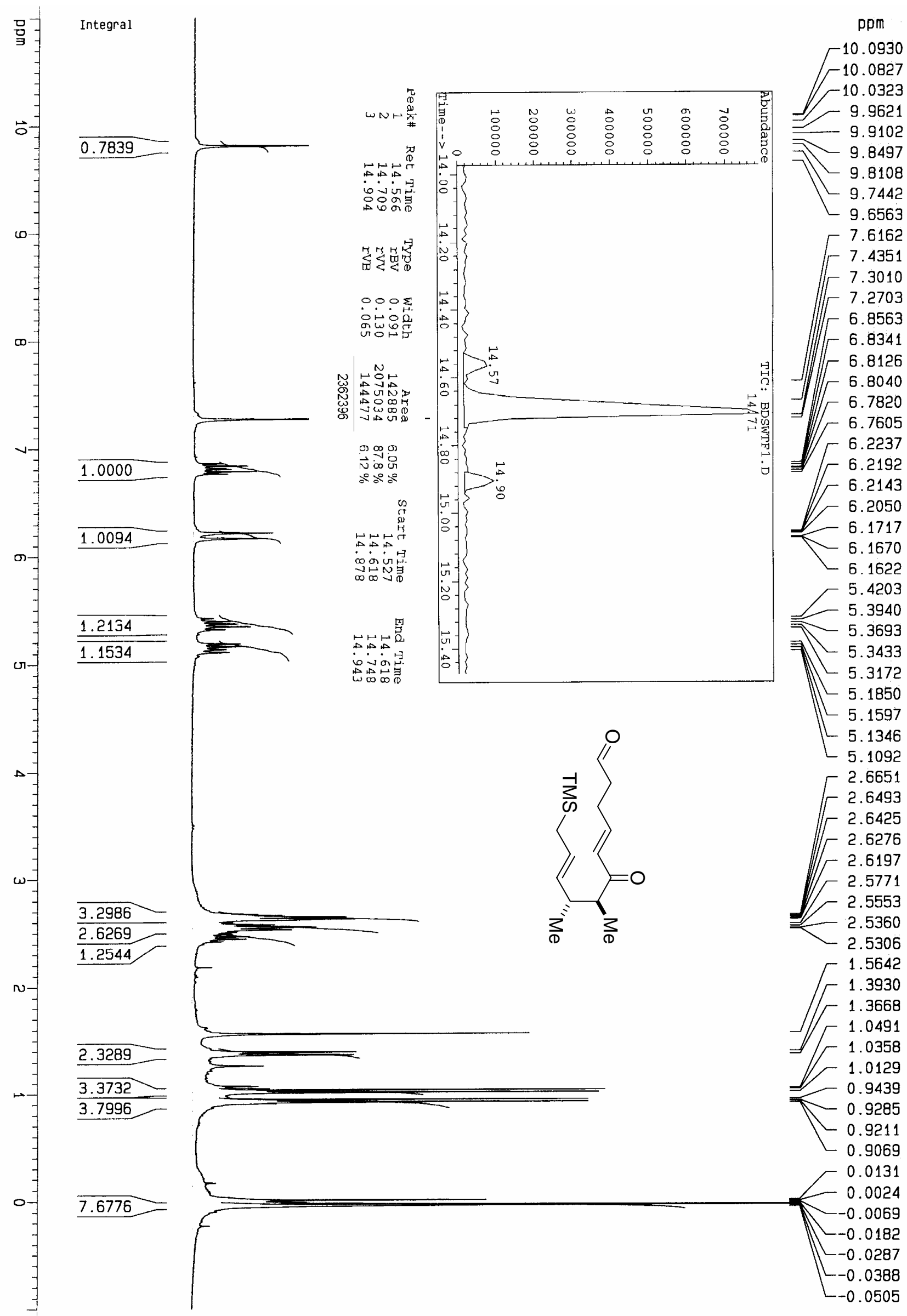



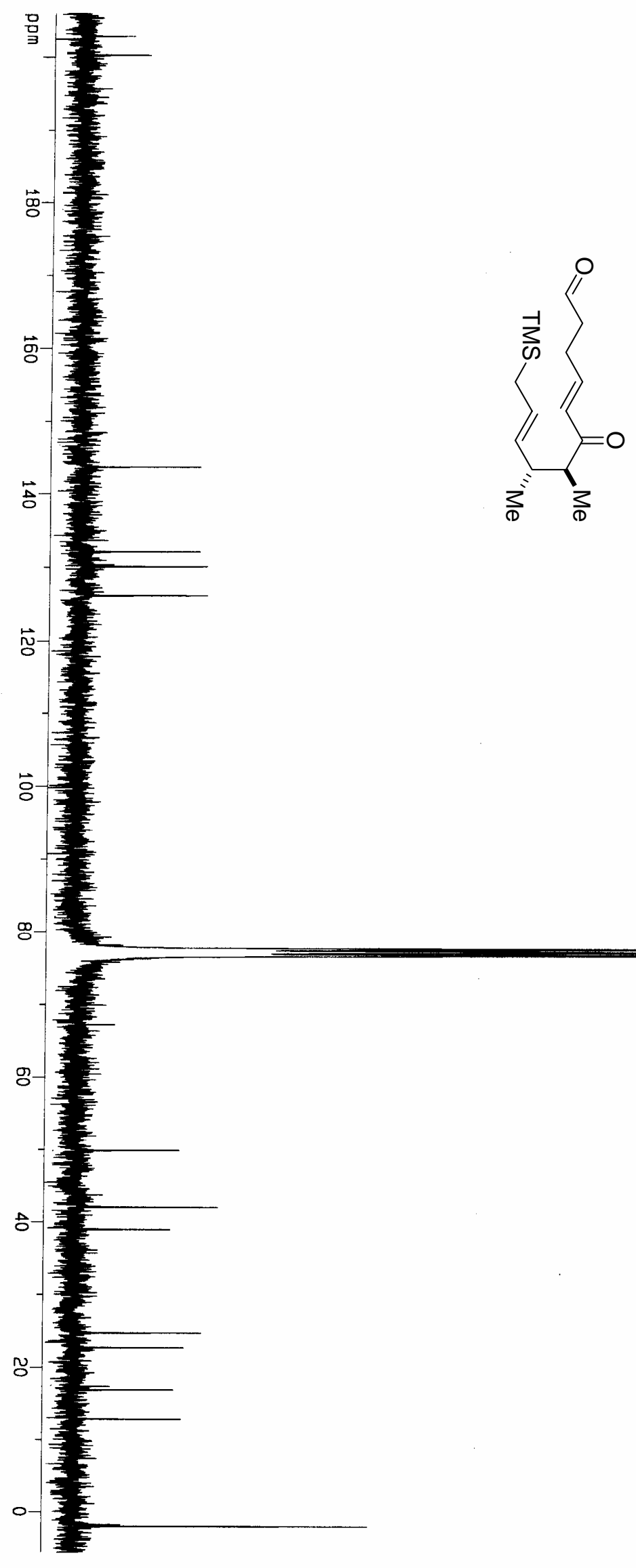

ppm
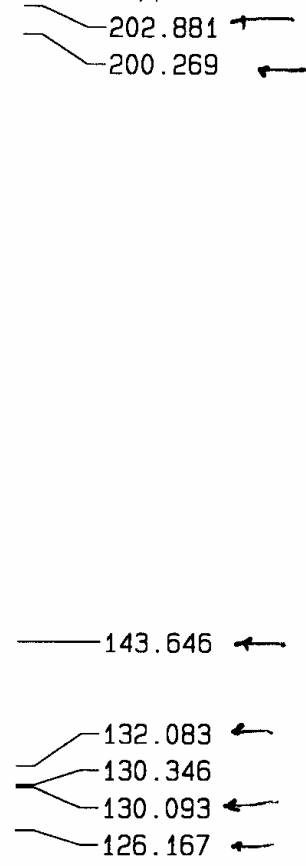

79.170

F 77.4717

$-77.194$

$-76.993$

$-76.569$

$-75.757$

69.901

$-67.237$

$49.859+$ 42.029 $38.968 \longleftarrow$

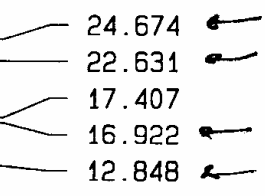

$-1.750$

$-1.978 \leftarrow$ 


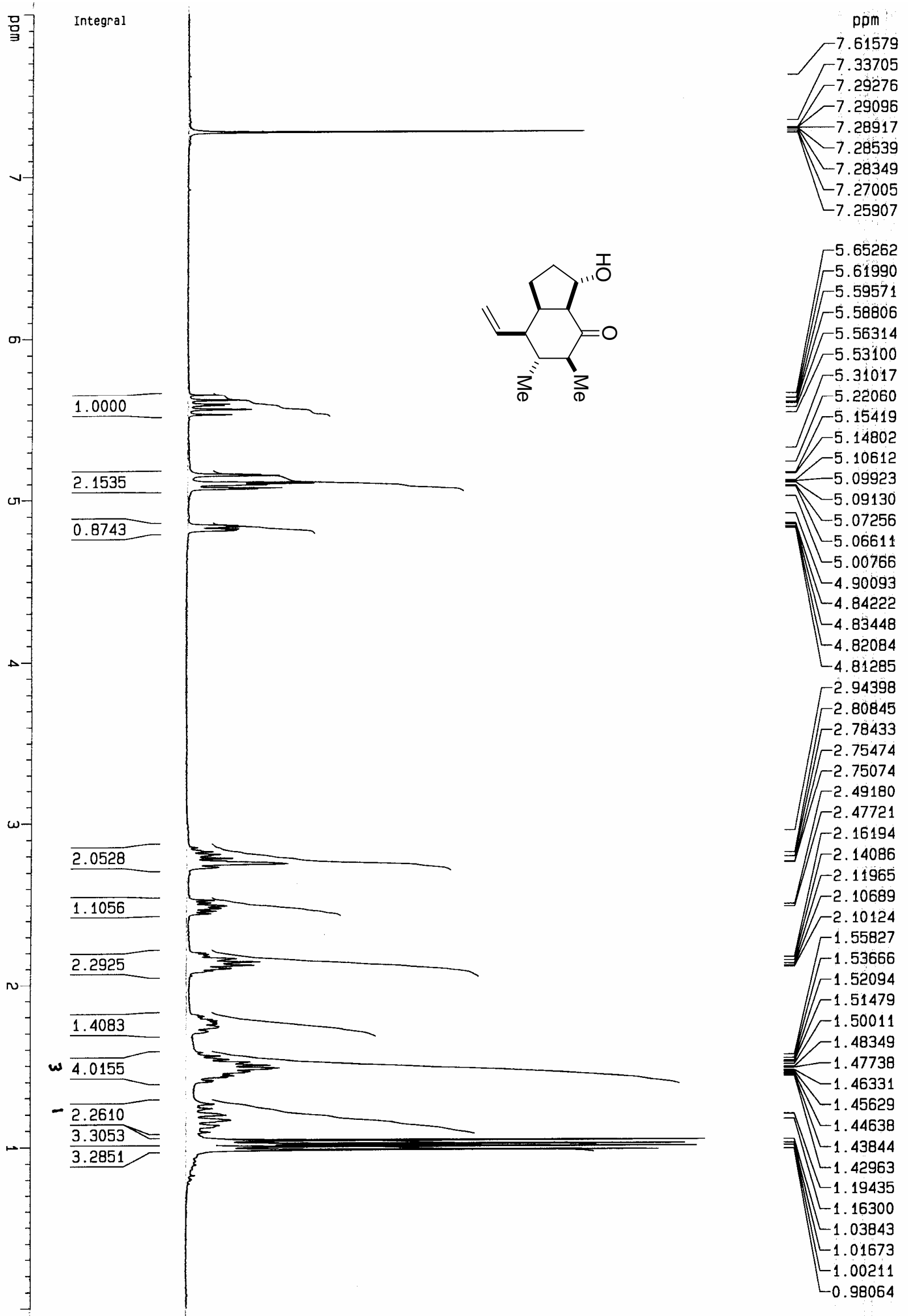




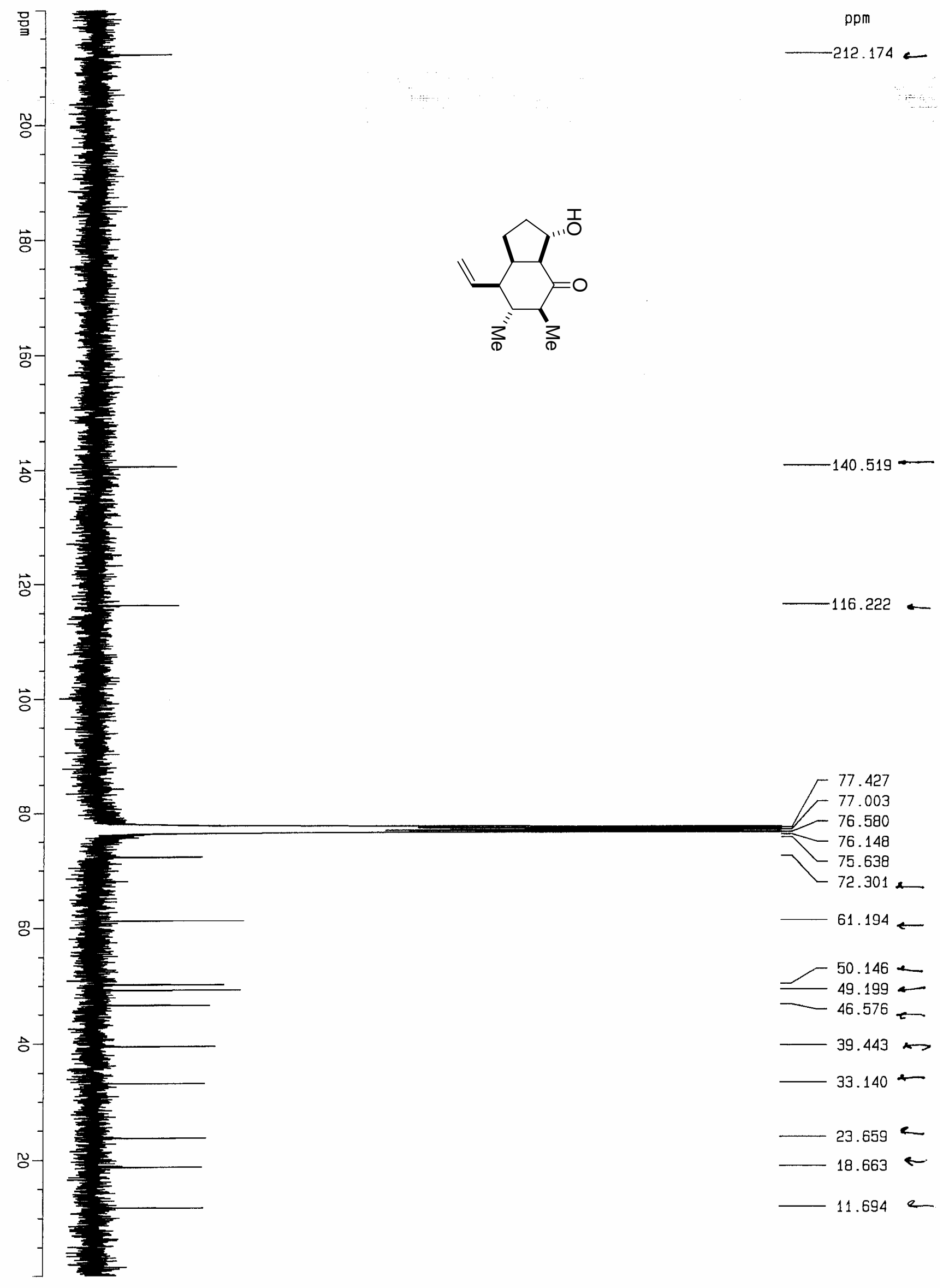




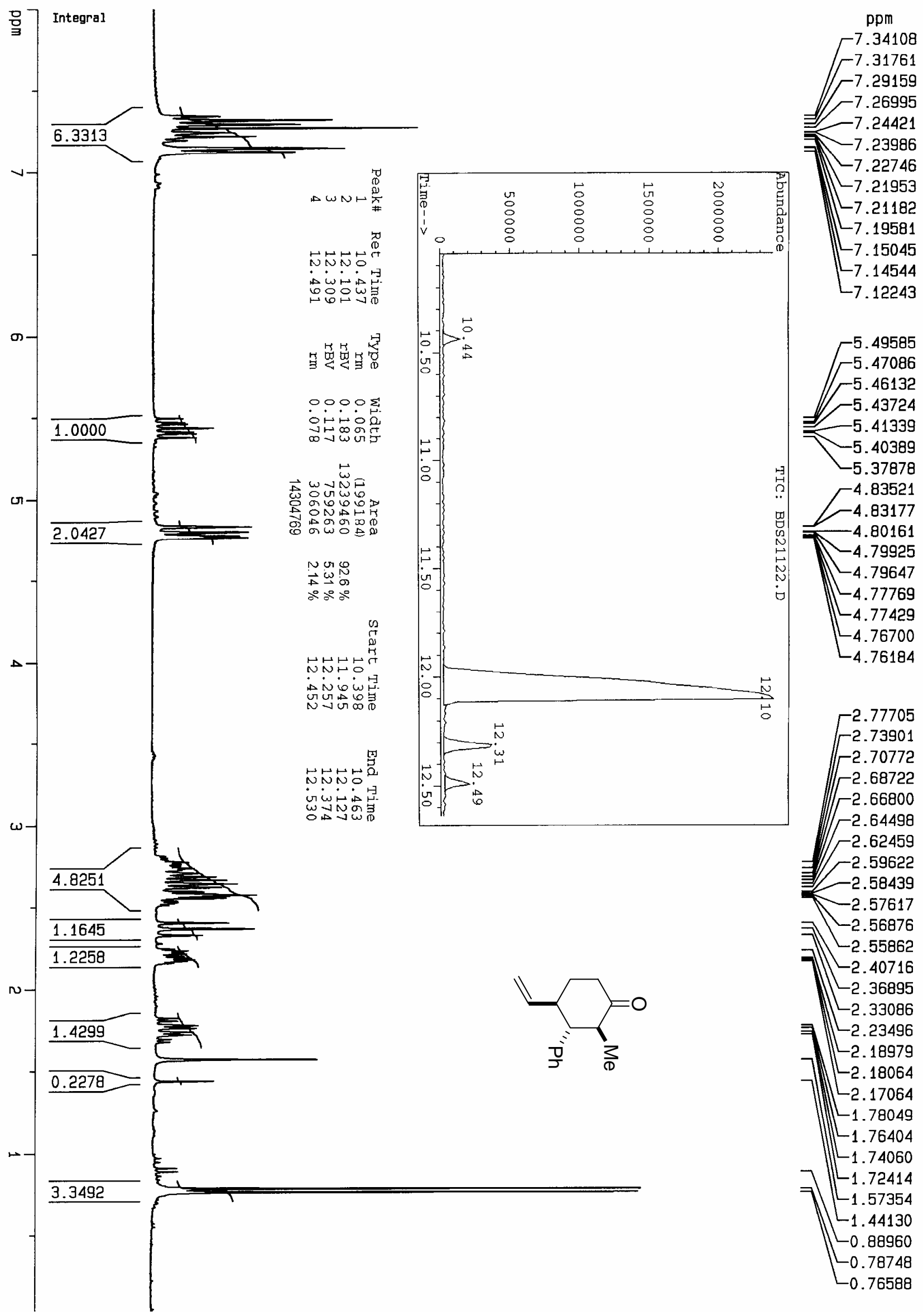




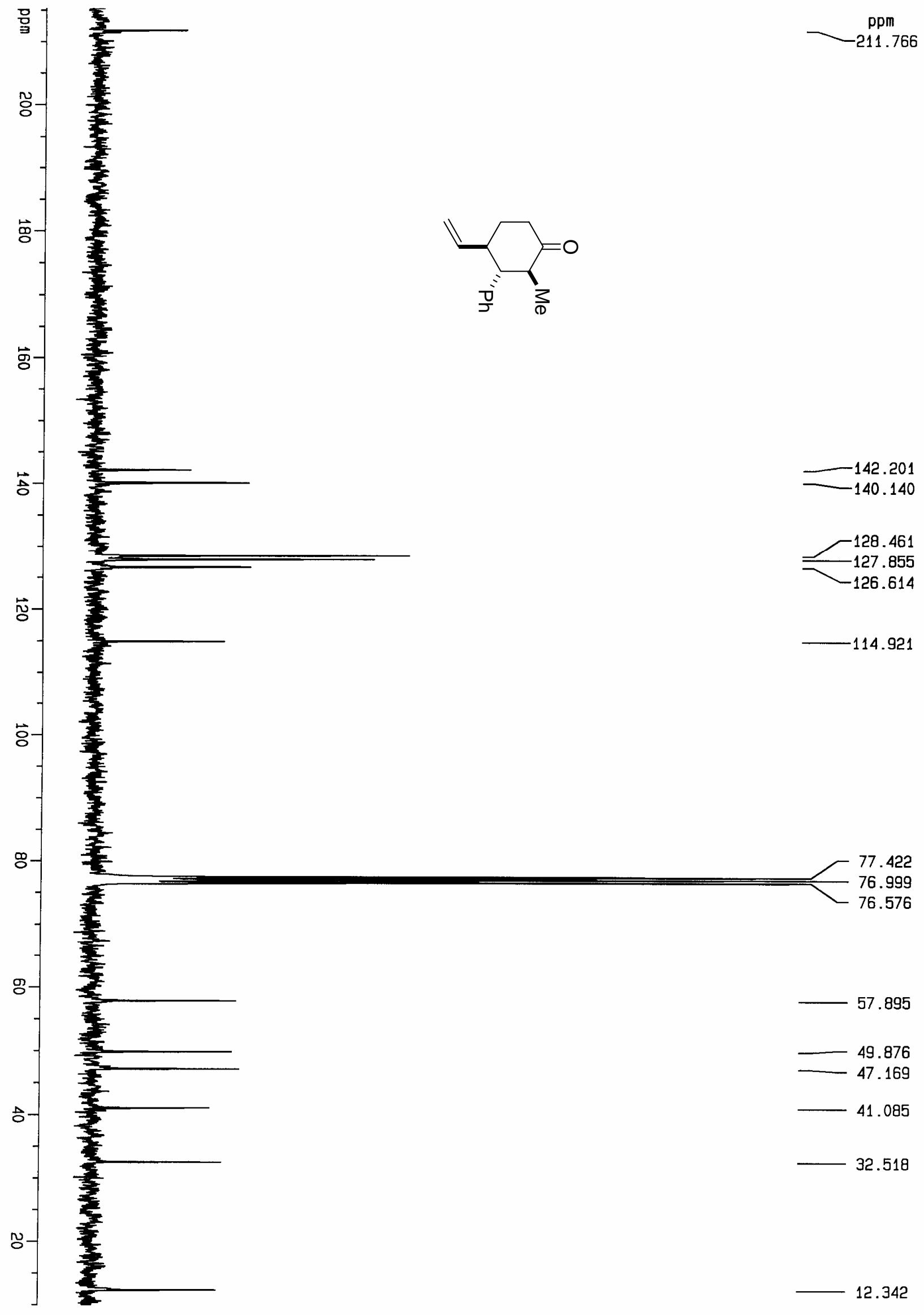




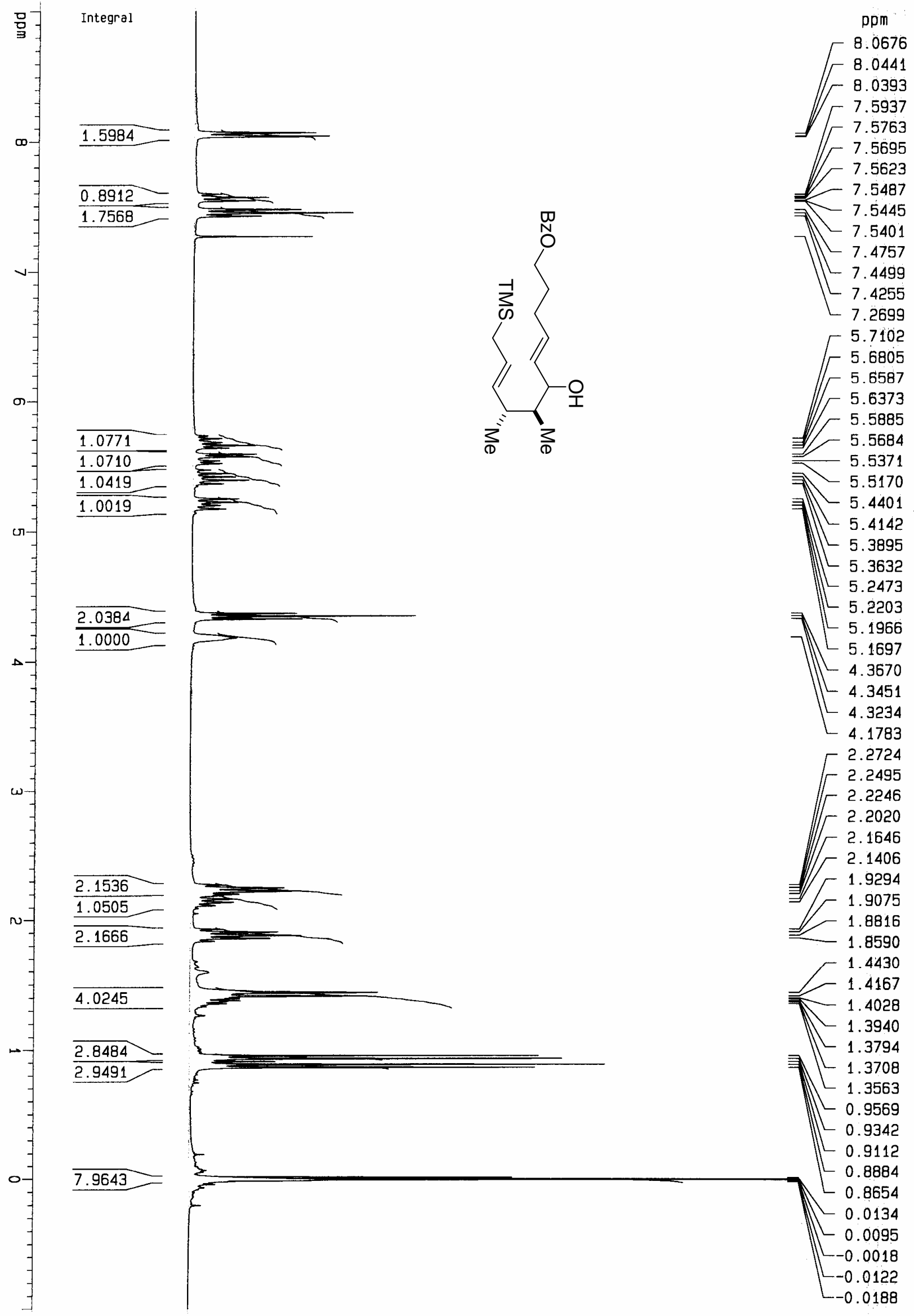



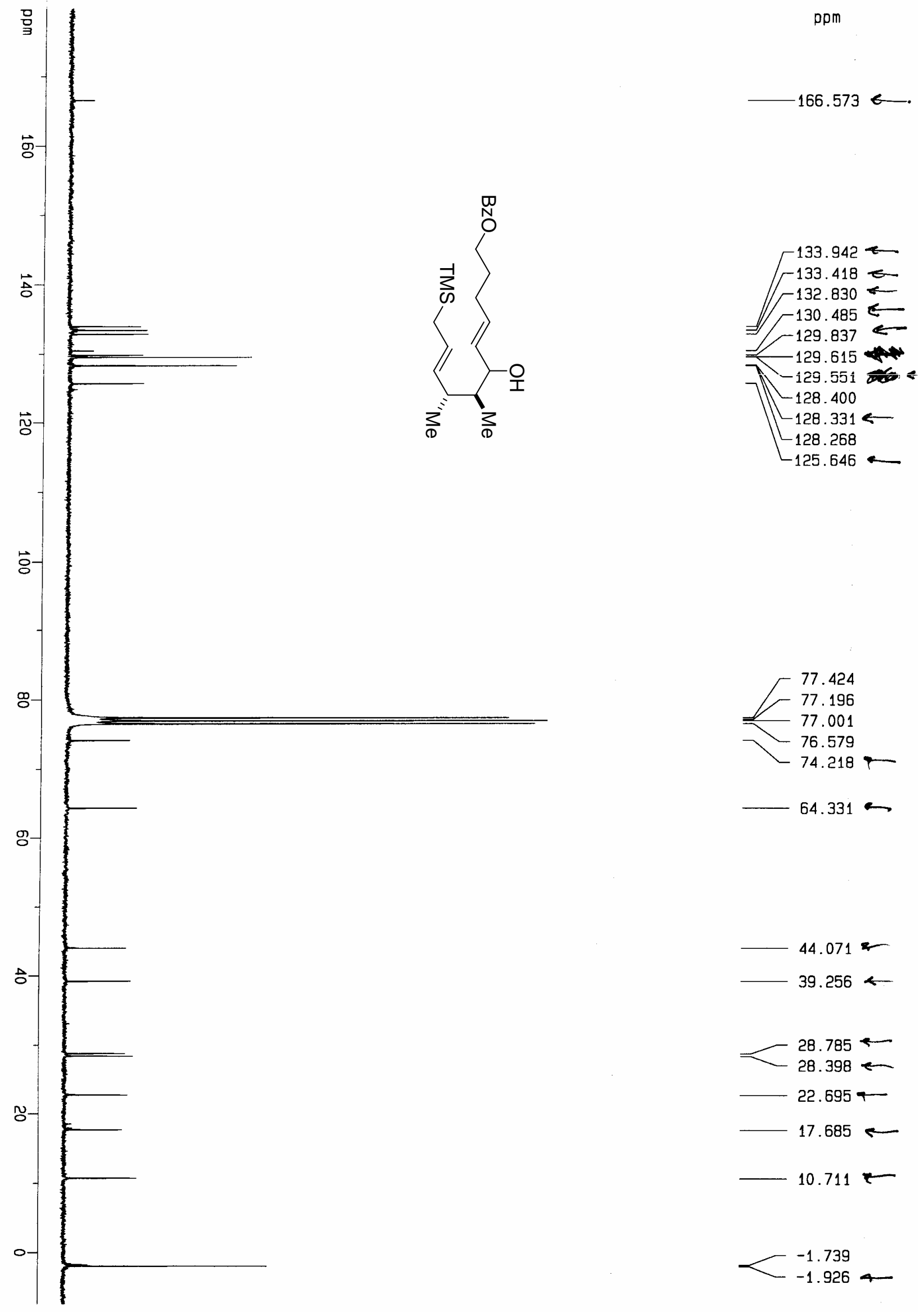

$44.071 \%$ $39.256 \longleftarrow$
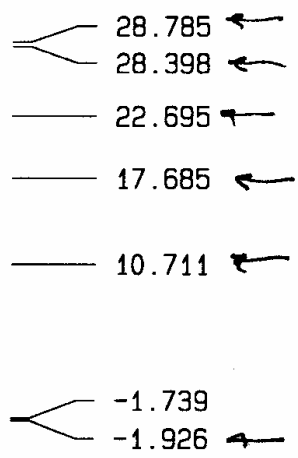


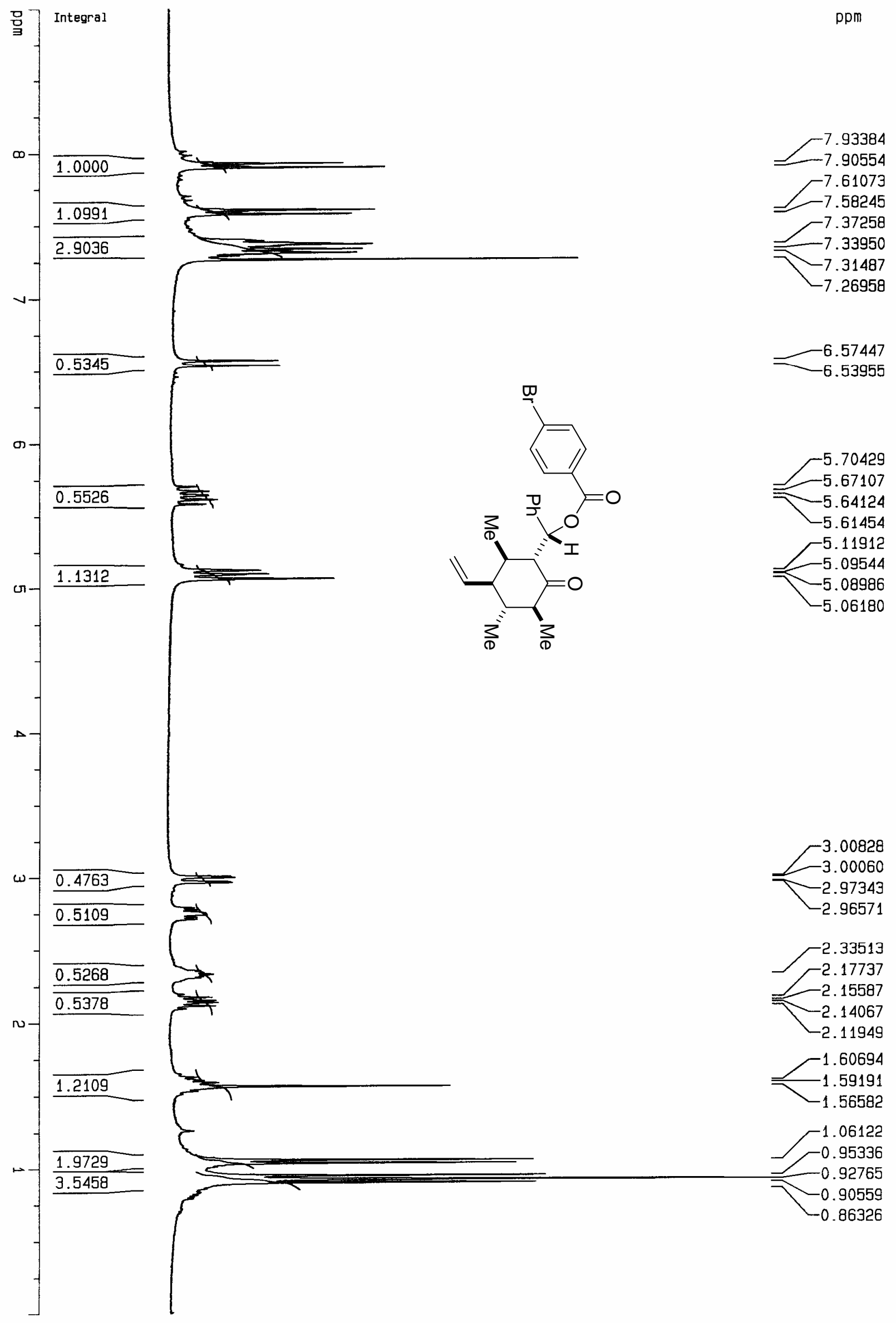




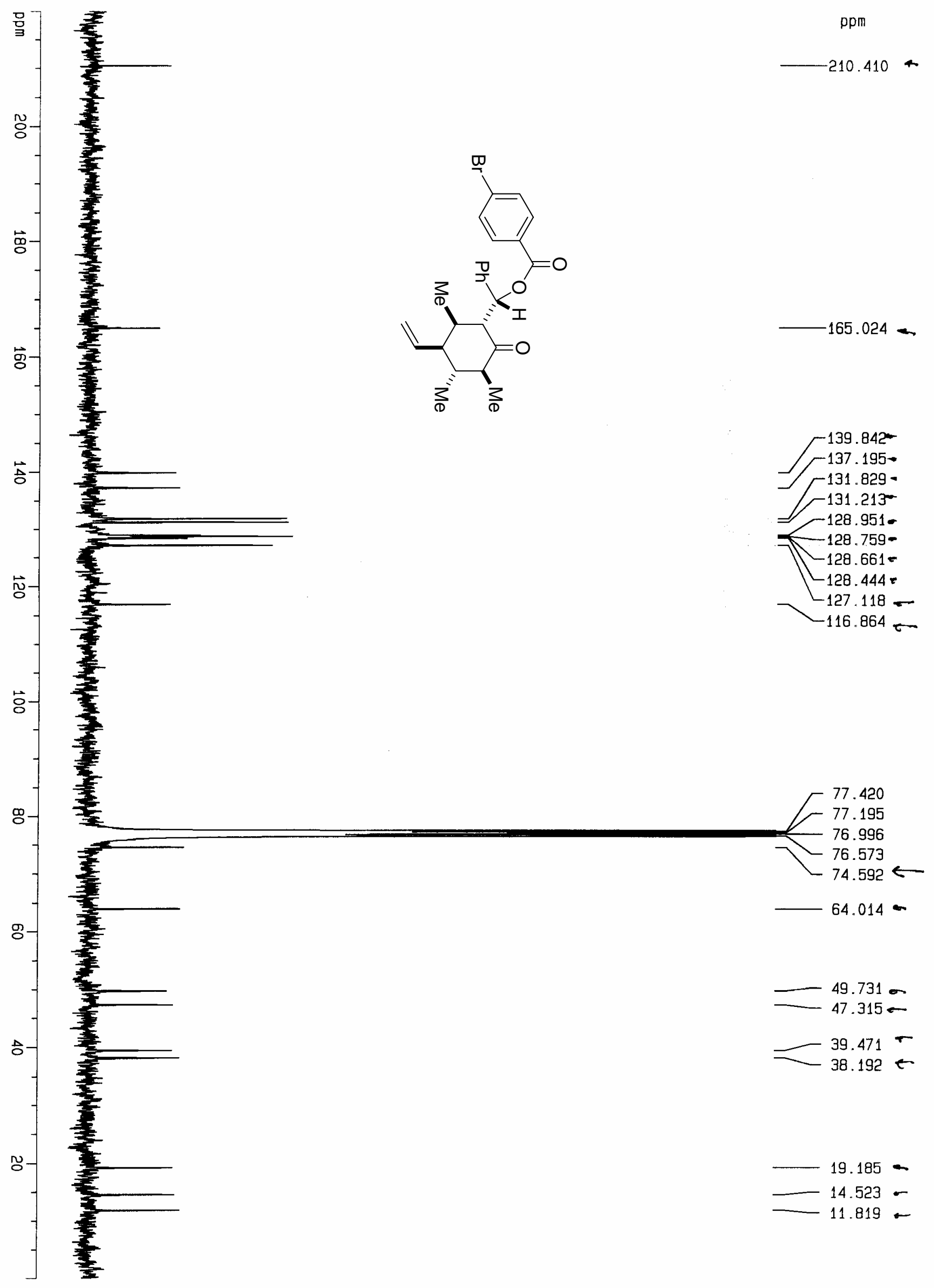




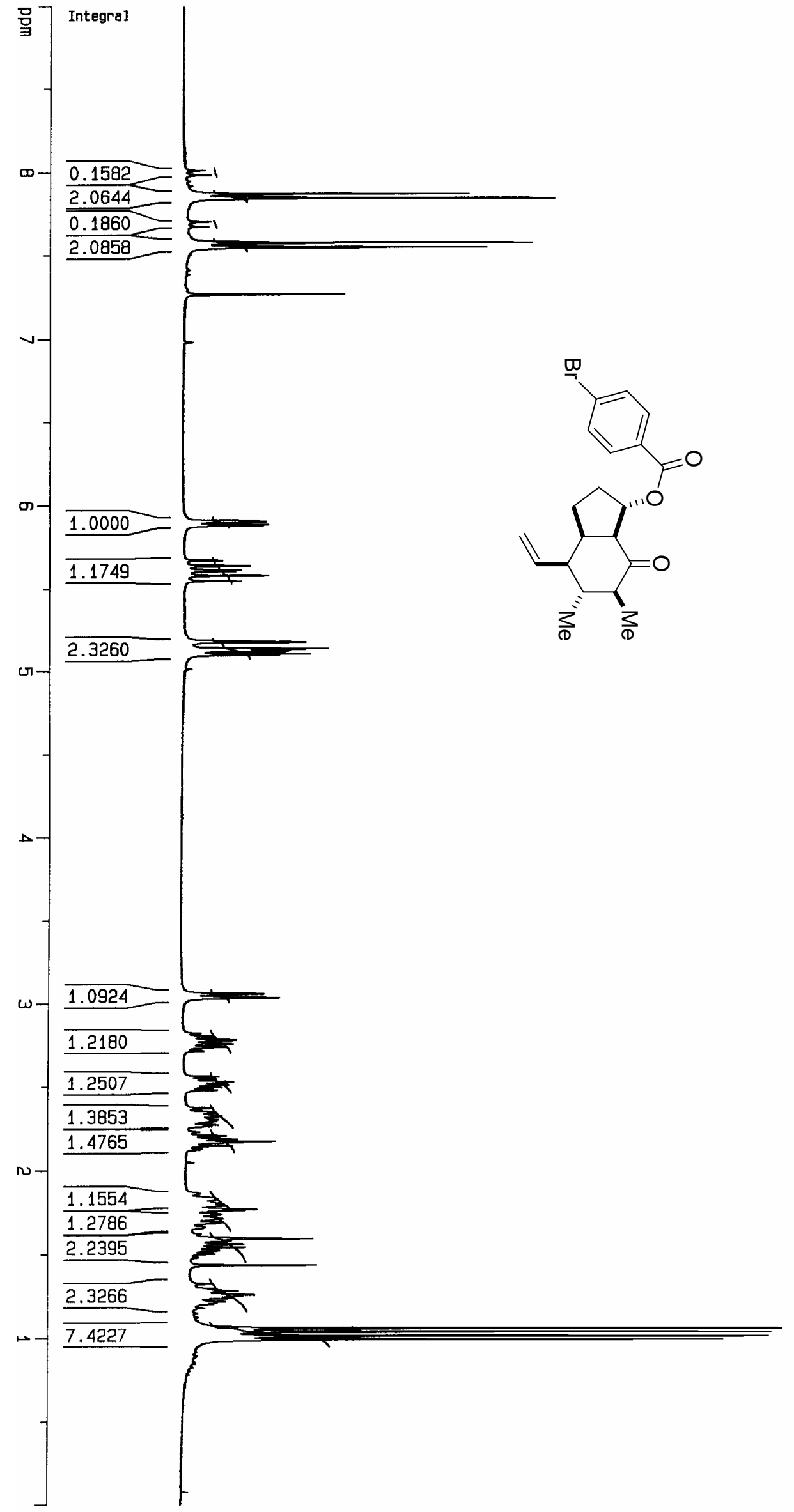

ppm

$-7.88148$

$-7.87347$

$-7.86777$

7.85166

$-7.84527$

$-7.83795$

$-7.58754$

$=7.57966$

$-7.57379$

$-7.55759$

$-7.55143$

$-7.54396$

$-7.27001$

5.90988

$-5.90344$

5.88618

5.88114

$-5.67139$

$-5.63872$

$-5.61452$

5.60680

$-5.58196$

$-5.54986$

$-5.18239$

$7-5.17633$

$-5.13717$

$-5.13070$

$-5.11941$

$-5.10361$

$\leftarrow_{5.09726}$

3.06031

$-3.03592$

-2.78353

$-2.76011$

$-2.74220$

-2.53133

-2.51414

-2.20814

-2.18961

-2.17681

-2.16974

$-2.15108$

$r^{1.79082}$

$-1.76692$

$\Gamma^{1.59450}$

$=1.57963$

$-1.56322$

1.54185

$-1.43858$

$\overline{M-1.28302}$

$-1.26180$

$-1.25531$

$-1.24169$

$-1.22147$

$-1.06298$

$-1.04142$

$-1.01564$ 


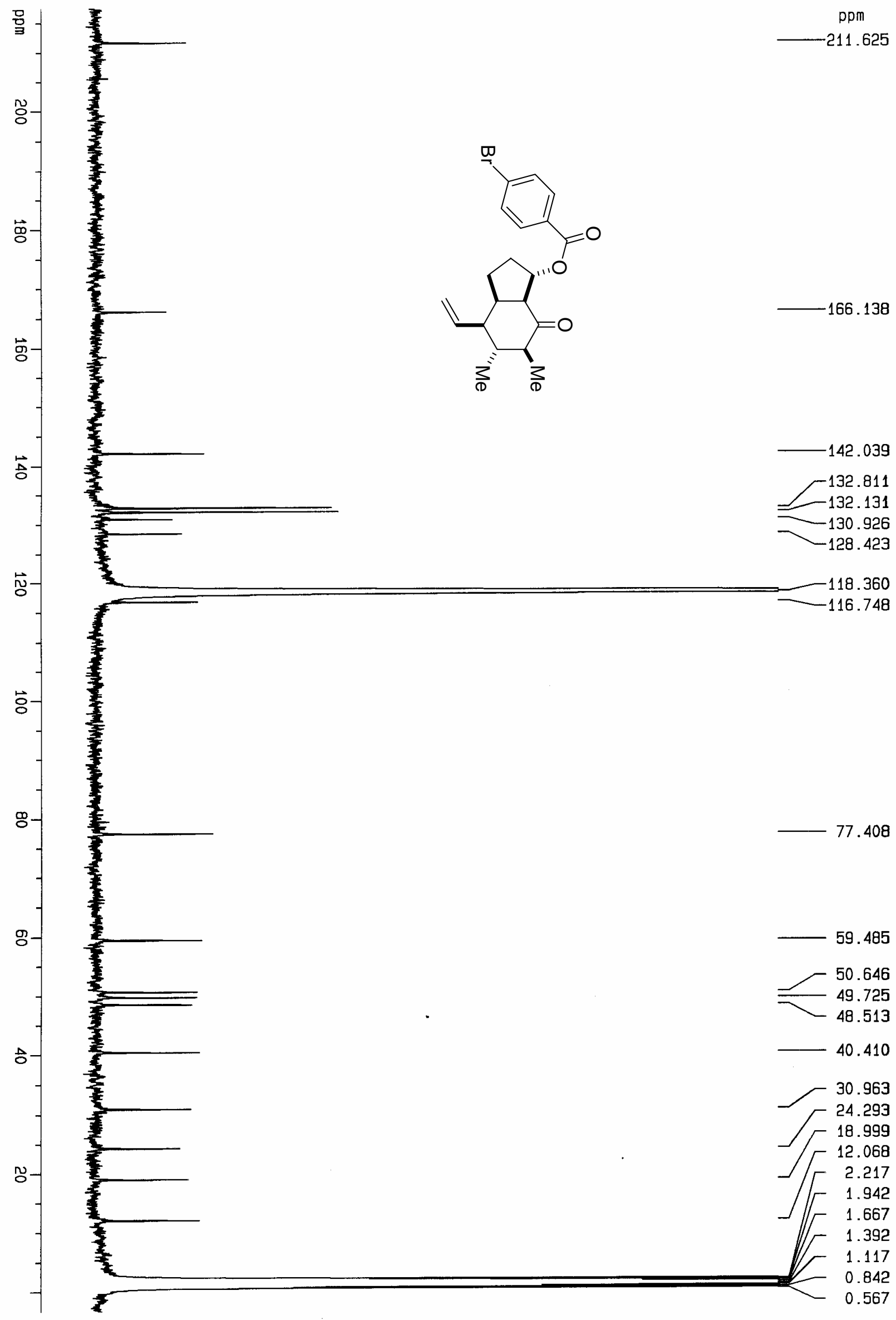




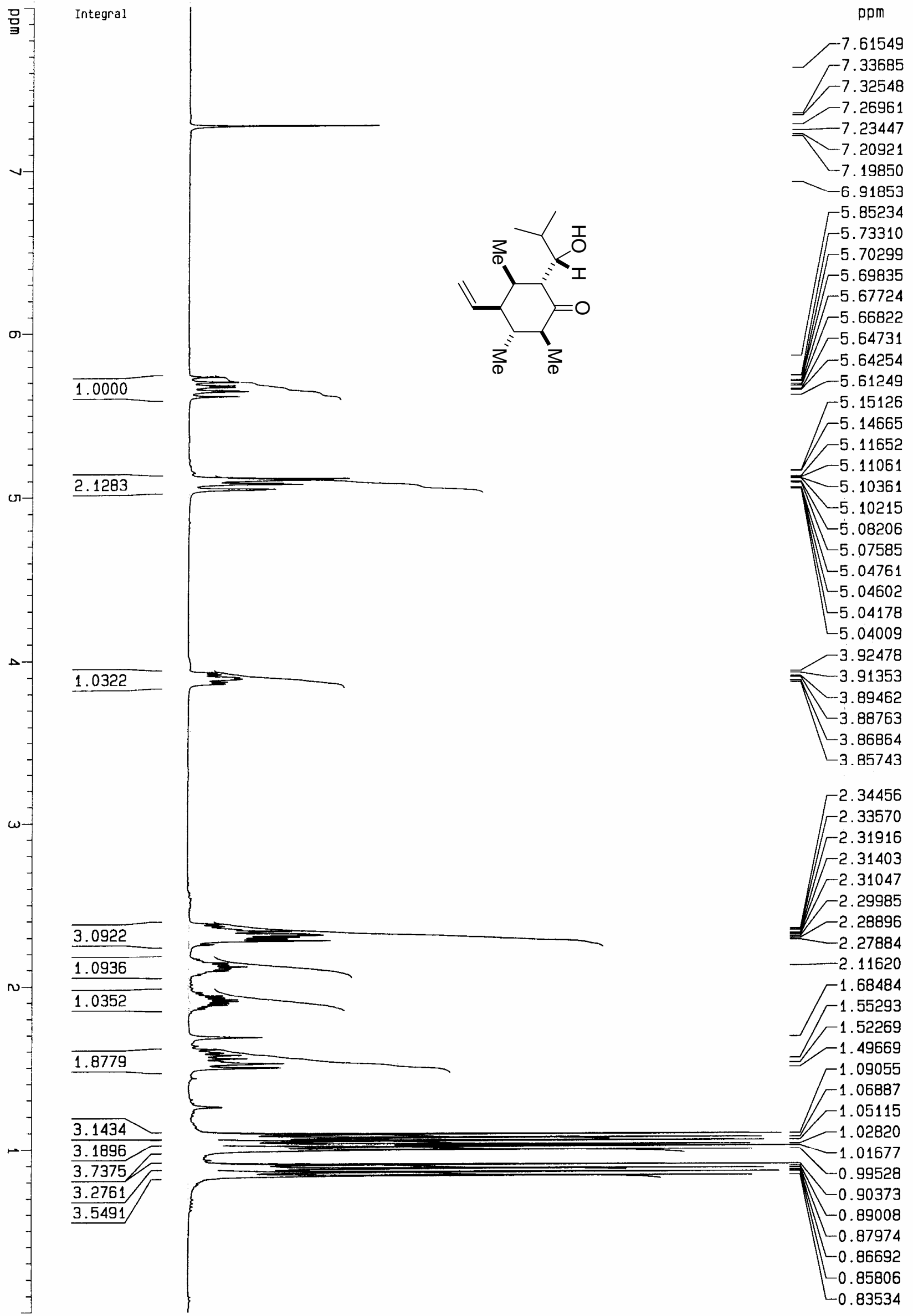




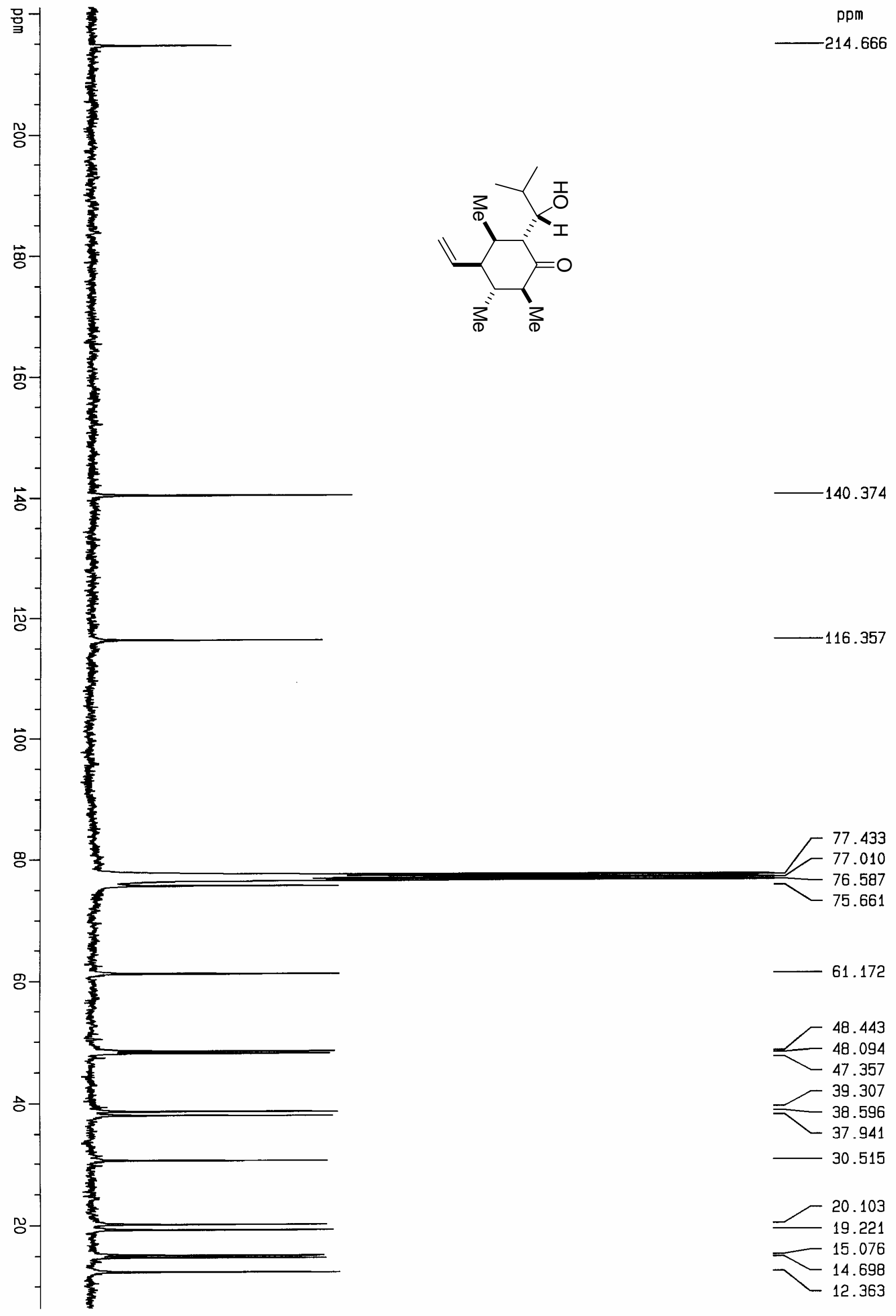

